\title{
ON THE THEORY OF POLARIZATION EFFECTS IN GAS LASERS
}

DAAN LENSTRA

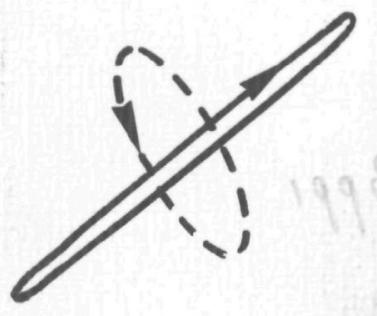


P1998

5201

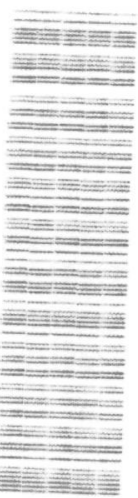

C 10068 15003 


\section{ON THE THEORY OF POLARIZATION EFFECTS IN GAS LASERS}

\section{PROEFSCHRIFT}

Ter verkrijging van de graad van doctor in de technische wetenschappen aan de Technische Hogeschool Delft, op gezag van de rector magnificus prof. dr. ir. F.J. Kievits, voor een commissie aangewezen door het college van dekanen, te verdedigen op woensdag 12 september 1979 te 16.00 uur

door

\section{DAAN LENSTRA}

natuurkundig doctorandus geboren te Amsterdam

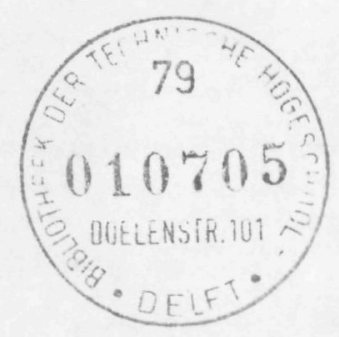

BIBLIOTHEEK TU Delft P 19985201

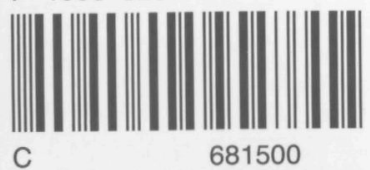


Dit proefschrift is goedgekeurd door de promotor Prof. Dr. W. van Haeringen 


\section{ON THE THEORY OF POLARIZATION EFFECTS IN GAS LASERS}

$\underline{\text { RATA }}$

17, Eq. (2-14): the 1.h.s. should read $\underset{=}{S}\left(\omega_{n, q}^{c}\right) \underline{\sigma}_{n, q}$

.23 , last two lines: replace $\underline{H}$ by $\underline{H}$

.24 , 4th term in the r.h.s. of Eq. (2-42) should read: $b_{-1,-1} E_{-1}\left|E_{-1}\right|^{2}$

?.28, r.h.s. of Eq. $(2-61)$ : replace $\beta_{2}^{\prime \prime}$ by $\beta_{2}^{\prime}$

p.58, last sentence: add the symbol $W$ between "two" and "matrices" p.113, third line: add \pm in front of $\Gamma_{\mathrm{a}} \cos 2 R$

p.123, second line of Eq. (C-1): the first term in the r.h.s. should read $n ; n, n, n$
$b_{ \pm 1 ; \mp 1, \pm 1, \mp 1}$ 
Aan de nagedachtenis van mijn vader 
De schrijver wil hierbij iedereen bedanken die heeft bijgedragen aan de verwezenlijking van dit proefschrift. In het bijzonder wil hij noemen Gérard Herman voor zijn deelname aan de onderzoekingen beschreven in de Hoofdstukken 5 en 6 en Susan Massotty voor het corrigeren van de Engelse tekst. 


\section{ABSTRACT}

Starting from a theory in which laser operation is described as the excitation of the natural-modes of an open-resonator system, the case of a Fabry-Pérot resonator with plane-parallel mirrors is investigated. It is shown that, in the case of weakly anisotropic mirrors, the natural-modes can be grouped in pairs, such that each pair corresponds to one scalar longitudinal mode. In that case, laser operation can and will be described in terms of these longitudinal modes, which then possess a vectorial field amplitude, in agreement with the usual formalism,

The polarization preference in a single-mode gas laser, operating on a transition between a singlet and a triplet state is investigated, taking into account different relaxation constants for the density matrix. A calculation of the effect on the relaxation constants due to interatomic collisions leads to the conclusion that theory and experiment are in good agreement with each other.

Equations describing the time evolution of the polarization parameters of both modes in a two-mode operating gas laser are derived. It is found as a general rule that mode-mode interaction results in modes whose polarizations are mutually orthogonal. An exception to this rule is found in the case in which the single-mode preference is for circular polarization. The influence of two specific mirror anisotropies is discussed.

The polarizing behaviour of a single-mode gas laser in the presence of (partly) applied, transverse magnetic fields is extensively dealt with. It appears to be essential to distinguish between the case in which the magnetic field is applied only to a fraction of the active medium and the case in which the magnetic field is uniform in the entire medium. This distinction is shown to lead to a satisfactory theoretical description of a certain class of experiments. In the case of two other experiments, good agreement with the theory is obtained, by assuming the presence of mirror anisotropies of acceptable kind and size. Finally, the influence of small anisotropies on the degree of polarization of a single-mode gas laser is theoretically investigated, when the latter is polarized by means of a transverse magnetic field. 
CONTENTS

CHAPTER 1 GENERAL INTRODUCTION

1.1 short review of literature

1.2 outline of the present study

CHAPTER 2 THE ELECTROMAGNETIC FIELD EQUATIONS

2.1 natural-modes of open resonators 9

2.2 the general laser equation 12

2.3 the laser equation for a Fabry-Pérot type of resonator with anisotropic losses 15

2.4 introduction of mode-polarization parameters 21

2.5 equations for the polarization parameters (single frequency case)

CHAPTER 3 RESPONSE OF A GAS TO AN ELECTROMAGNETIC FIELD $(j=1 \rightarrow j=0$ TRANSITION CASE $)$

3.1 derivation of the equations of motion of expectation values

3.2 solution of the equations of motion 37

3.3 the polarization response

CHAPTER 4 THE $1 \rightarrow 0$ TRANSITION LASER

4.1 behaviour of the polarization parameters

4.2 calculation of $\lambda_{J}$ and $\lambda_{Q}$

CHAPTER 5 INTERACTION OF THE LASER FIELD WITH THE ACTIVE MEDIUM (GENERAL CASE)

5.1 derivation of the equations

5.2 third-order solution of the equations

5.3 third-order expression for the polarization response 
CHAPTER 6 POLARIZATION EFFECTS IN SINGLE-MODE AND TWOMODE GAS LASERS $\quad 77$

6.1 derivation of the two-mode equations 77

6.2 behaviour of the polarization parameters (isotropic loss tensor) 84

6.3 the influence of a weak cavity anisotropy 90

CHAPTER 7 MODE-POLARIZATION IN TRANSVERSE MAGNETIC FIELDS

(SINGLE-MODE CASE) 99

7.1 transverse magnetic field applied to the entire resonator (isotropic loss tensor) 100

7.2 transverse magnetic field applied to a fraction of the resonator (isotropic loss tensor) 105

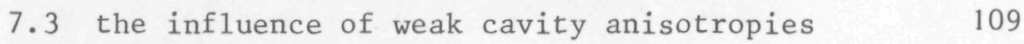

7.4 polarization effects in large transverse magnetic fields

APPENDICES A. calculation of natural-modes in the onedimensional model 119

B. the loss tensor 121

C. calculation of the $\alpha, \beta, \gamma$ and $\delta$ coefficients 123

D. approximate solution of equation (7--2) 125

$\begin{array}{ll}\text { LIST OF CONVENTIONS AND FREQUENTLY USED SYMBOLS } & 127\end{array}$

1 conventions 127

2 Latin symbols 128

3 Greek symbols 130

REFERENCES 


\section{GENERAL INTRODUCTION}

This study is devoted to a theoretical analysis of polarization phenomena in a gas laser. 'In this introduction we will first recall some general laser features. We then give a brief survey of the existing literature on the subject, followed by an outline of the present study.

A device that possesses the ability to amplify light by stimulated emission of radiation is commonly called a laser, where the word "LASER" is an acronym for "Light Amplification by Stimulated Emission of Radiation". In this connection the word light stands for electromagnetic radiation whose frequency lies somewhere between the near-infrared and the ultraviolet. Of prime importance in a laser system is the presence of a certain region, enclosing material in which amplification of radiation by stimulated emission can take place. Though it is very well possible to observe amplification in the amplitude of a travelling light wave, one of ten encloses the material in a resonator, which serves as an oscillator for electromagnetic waves. In this respect there is some similarity with a classical sustained oscillator [1], in which the oscillator is an RLCcircuit, while the amplifier can be a triode tube or a transistor. This system will produce oscillations at a frequency close to the eigenfrequency of the oscillator, just as a laser that oscillates in one or more frequencies close to the eigenfrequencies of the resonator.

One of the conditions necessary for stimulated emission is the existence of a "population inversion" in the material. By this we mean that, of the atoms or molecules involved in the amplification process, a majority occupies a certain upper energy level, while the rest occupies a given lower level, the energy difference between the two levels being about equal to $\hbar$ times the laser frequency. This condition is crucial for obtaining laser operation. A material that has a population inversion in the above sense is often referred to as "active" material. In contradistinction, a "passive" material has no population 
inversion. As materials are usually found on earth in their passive manifestations, some "pumping process" has to be used to achieve population inversion. This notion reflects that energy has to be pumped into the material to make it active. Pumping can be achieved by several methods; the choice depends on the specific properties of the material [2].

In the present study we will be concerned with gas lasers in which stimulated emission takes place in a spectral line belonging to a neutral or ionized atom or molecule. The population inversion is usually achieved by a permanent electric discharge current in the gas. In most cases, the resonator consists of two strongly reflecting, approximately plane-paralle1 or slightly curved mirrors, while the active gas occupies a region in between the mirrors.

In view of apparent discrepancies between experiments and existing theory, we will start by reformulating the general theory of gas-laser operation, which allows for a description of the properties of the electromagnetic field. Special emphasis will be put on the state of polarization of this field. As a matter of course the theory includes a detailed description of the interaction between radiation and the active gas. This will involve the study of the time evolution of atomic observables under the influence of a classical, i.e., nonquantized, radiation field. This approach, known as the "semi-classical" approach, leads to a correct description as long as a not too low intensity of the radiation field is considered [3,4]. Consequently, no attempt will be made to include neither the operation of a laser close to threshold nor its noise properties.

In the theory to be developed we will include the possibility that the active gas, or only a part of it, is subject to a magnetic field. In that case, at least one of the energy levels involved in the laser transition is split up in energy (Zeeman-splitting). As is well known, this may give rise to interesting polarization effects in the output of the laser. Especially, the effects due to transverse magnetic fields, i.e. fields that are applied to the medium perpendicularly to the propagation vector of the electromagnetic field, will be discussed in great detail, leading to the resolution of a number of discrepancies. 


\section{1 short review of Ziterature}

The first succesful attempt to realize laser operation in a gas was reported in 1961 by Javan et a1. [5], They succeeded in obtaining continuous laser operation at five spectral lines of Neon in the near-infrared. The strongest oscillation observed was at $1.153 \mu \mathrm{m}$. The design of their laser system was based upon ideas set down in 1958 in an article by Schawlow and Townes [6]. The resonator was of the Fabry-Pérot type, consisting of two plane-parallel mirrors of large reflectivity placed at a distance of $0.8 \mathrm{~m}$ from each other.

Soon after the first succesful performance, many investigations were started to study the properties of lasers, both experimentally and theoretically. An early, intuitive theory of the nonlinear interaction between the intense laser field and the active medium in the laser was given by Bennet [7]. An article by Statz et a1. [8] reports on certain features observed in the output of a He-Ne laser, which they claimed were caused by the earth's magnetic field. According to these authors the laser field was linearly polarized, while the plane of polarization rotated at a definite frequency. The explanation of this effect can indeed be based on the presence of a D.C. magnetic field along the laser axis. Due to the Zeeman-splitting of the atomic energy levels involved, the transition frequencies of right- and left-handed circularly polarized light are no longer precisely equal, and this results in slightly different operation frequencies. If both circular components have equal magnitude, this manifests itself in the phenomenon described above.

An early but incomplete attempt to gain insight into the nonlinear (or saturation) effects in the resonant absorbtion of several simultaneously oscillating fields by a gas was made in 1962 by Tang \& Statz [9]. Their approach is based on the use of the density-matrix formalism to describe the quantum mechanical state of the atoms. During the years 1963 to 1965 a large number of articles appeared, dealing with the effect of D,C. magnetic fields on the output of a gas laser (see, for instance, [10] through [22]).

The theory of the gas laser given by Lamb [23], which appeared in 1964, was a large step forward. In his theory the atoms are treated quantum 
mechanically as objects with two nondegenerate levels only (two-level system), while the electromagnetic field is treated as a classical scalar field. Lamb's theory can be succesfully applied to lasers in which the electromagnetic field has a prescribed state of polarization, such as in Brewster-window lasers. There is a restriction to intensities that are neither too low nor too high. The first condition circumvents the necessity of quantizing the laser field, the second justifies the assumption that on1y third-order nonlinearities in the electromagnetic response of the medium need be taken into account. Several laser features can be succesfully accounted for in this way, as long as no external magnetic fields are applied. However, apart from questions concerning magnetic-field effects in gas lasers, the fundamental shortcoming is that effects connected with the vectorial character of the electromagnetic field, such as polarization effects, cannot be treated in the framework of Lamb's theory.

It was observed by several authors that the $1.153 \mu \mathrm{m}$ Ne transition mode in a He-Ne gas laser is linearly polarized [8, 9, 24], A crucial experiment by De Lang et al. [24] on a planar single-mode He-Ne 1.153 $\mu \mathrm{m}$ 1aser in the absence of magnetic fields provided evidence that the medium causes the laser mode to be linear. Using a phenomenological model for the gaseous medium, these authors showed that this polarization preference is due to anisotropic saturation of the medium. By extending Lamb's theory, taking into account the degeneracy of the atomic energy levels and by considering a vectorial electromagnetic field, Polder \& van Haeringen [25] showed that the kind and size of such a saturation-induced polarization preference is in fact determined by the angular-momentum quantum numbers of the two energy levels. Their theory indeed shows a preference for linear polarization for the $1.153 \mu \mathrm{m}$ Ne transition, in agreement with the above experiment [24]. Moreover, a prediction was made concerning a class of transitions for which a preference for circular polarization should be observed. This prediction was soon followed by an experimental verification by De Lang and Bouwhuis [26].

However, for the case of the $j=1 \leftrightarrow j=0$ and the $j=1 \rightarrow j=1$ type of transitions ( $j$ denotes the angular-momentum quantum number), the theory predicted the absence of a polarization preference, which was in 
disagreement with an experimentally observed weak, but definite, preference for circular polarization [26]. An explanation for this discrepancy was given in 1967 by Polder \& Van Haeringen [27] by relating the polarization preference in these cases to collision-induced relaxation phenomena of excited atorhs. These relaxation phenomena had already been theoretically investigated in 1965 by Omont [28] and by D'Yakonov \& Perel [29], but not in connection with the above-mentioned polarization phenomena.

Since the appearance of Lamb's theory in 1964, several extensions have been made in order to include magnetic-field effects. We mention the work of Durand [30] and Culshaw \& Kannelaud [31] who treated the case of a $j=1 \rightarrow j=0$ transition in the presence of transverse [30,31] and axia1 [31] magnetic fields. A general theory of laser operation in the presence of axial or transverse magnetic fields was developed by D'Yakonov \& Perel in 1966 [32], however without giving applications.

A number of experiments had made it evident that not only saturation effects in the medium but also small anisotropies in the reflectivity of the mirrors substantially influence the state of polarization of the laser field [12,34-37]. De Lang [36] has given a phenomenological theory leading to the conclusion that the state of polarization of a laser mode is determined by (i) the anisotropy in the nonlinear response of the active medium if no external magnetic fields are present, (ii) the effects of externally applied magnetic fields and (iii) the anisotropies present already in the empty resonator. Pelikan [38] treated laser operation in the presence of an axial-magnetic field $(j=1 \rightarrow j=0$ transition) and in the presence of a special kind of cavity anisotropy. A more general treatment, including arbitrarily degenerate levels, weak axial magnetic fields and an arbitrary kind of small cavity anisotropy, was given by Van haeringen [39].

At the end of 1967, two articles by Sargent et al. [40, 41] reported on the development of a computer program by which laser operation could be simulated. The underlying theory is in fact similar to that in Reference [39], but includes the effects of magnetic fields of any magnitude and direction. A number of results of the computer study are presented in their second paper [41]. Although a comparison of these results with a 
set of experiments, viz. [13, 22, 42, 43], seems at first sight to give qualitative agreement, a more detailed examination of the theoretical and the experimental results, especially in the transverse magnetic-field cases, brings several discrepancies to light [44, 45].

More recently, the application of transverse magnetic fields in gas lasers has attracted renewed interest [46 - 50], among others because of its ability to force the laser to produce stabilized, linearly polarized light.

\section{2 outline of the present study}

As we wish to ground the theoretical description of the gas laser on the theory of passive open resonators, Section 2.1 will be devoted to a discussion of open-resonator features that are relevant for the description of the gas laser. Laser operation can be described as an excitation of natural-modes during time intervals much larger than the characteristic decay times of those open-resonator "eigen"-modes. An interesting exception to this general rule is explicitly dealt with. Isotropic as we11 as anisotropic radiation loss is accounted for in a consistent way when this approach is followed. In Lamb's theory as well as in later extensions, viz. [30, 32, 38, 39, 40], radiation loss is phenomenologically introduced.

A general laser equation describing the time evolution of laser modes will be derived and will be applied to a Fabry-Pérot type of resonator. It is shown, by using a one-dimensional resonator model in which one of the mirrors may have weakly anisotropic reflection properties, that the laser equation is reduced to a more familiar form. The same result can be obtained if both strongly-reflecting mirrors are weakly anisotropic. The equation is then transformedinto a set of equations giving the time evolution of parameters which describe the state of polarization of each mode. In these equations, coefficients occur which are to be determined from a detailed form of the electric polarization response of the active gas to the electric field.

In Chapter 3 these coefficients will be calculated for the case of a $j=1 \rightarrow j=0$ type of transition in the absence of magnetic fields, while the 
electric field is of single frequency. This case is not only interesting as an introduction to the more general and complex cases to be handled in Chapter 5, but the separate treatment enables us to elucidate the crucial role played by relaxations within the $j=1$ multiplet of excited atoms. In Chapter 4 we will apply the obtained results to the problem concerning the medium-induced polarization preference in a single mode $j=1 \rightarrow j=0$ laser. A model calculation on collision-induced relaxation based on References $[28,29]$ supports the conclusion that theory and experiment are in good agreement with each other.

In Chapter 5 the response of a gas to a multi-mode electric field, in the presence of an externally applied magnetic field, will be calculated for all kinds of electric-dipole-allowed transitions, leaving out the effects of interstate relaxations.

In Chapter 6 the medium-induced polarization preferences in magneticfield-free gas lasers are discussed for both single-mode and two-mode operation. In the latter case it is found that mode-mode interaction generally leads to a preference for mutual orthogonality of the modes. An interesting exception to this rule is also discussed. In addition, the effect of weakly anisotropic radiation losses on the polarization of the laser mode(s) will be discussed for two special kinds of anisotropy.

The subject of Chapter 7 will be the polarization behaviour of singlemode gas lasers in transverse magnetic fields. Our theory will be confronted with the earlier-mentioned set of experiments involving transverse magnetic fields, i.e., $[13,22,43]$. It will be shown that a magnetic field which is only partially applied to the laser medium leads to predictions of the polarization behaviour quite different from those concerning a field that is uniform in the entire active medium. A satisfactory description of certain experimental observations [22] follows if the assumption is made that the applied transverse magnetic field was in fact inhomogeneous. In two other cases, i.e., [13,43], good agreement between theory and experiment is obtained by assuming the presence of cavity anisotropies of acceptable kind and size. Finally, the influence of small cavity anisotropies on the output of a single-mode gas laser, which is polarized by means of a transverse magnetic field, will be dealt with in some detail. 


\section{THE ELECTROMAGNETIC FIELD EQUATIONS}

\section{1 natural-modes of open resonators}

We will define a resonator to be a spatially bounded structure consisting of a finite number of material bodies $M_{1}, \ldots, M_{m}$, later on to be identified with mirrors, which are embedded in free space (see Figure 2.1). In this respect a restriction will be made to mirror materials responding passively and linearly to electromagnetic fields. In some treatments so-called "closed" resonators have been introduced, for which electromagnetic field distributions that vanish identically outside a certain finite region are considered. However, in principle there exist no resonator systems satisfying this qualification. Therefore, all resonators should be considered as "open".

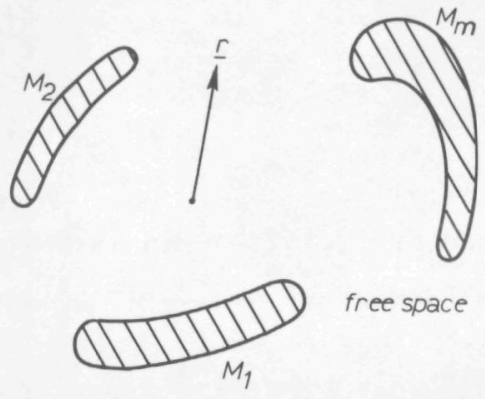

Fig.2.1 Open resonator consisting of a finite number of material bodies $M_{1}, \ldots, M_{m}$, embedded in free space

Loosely speaking, open resonators offer the possibility of localizing electromagnetic energy in space for a shorter or longer time, depending on the actual configuration of $\mathrm{M}_{1}, \ldots, \mathrm{M}_{m}$ and the particular form of the field. Open resonators show resonant behaviour. This is a direct consequence of the existence of a special series of solutions (free oscillations) of the corresponding source-free electromagnetic field equations. A free oscillation has outgoing wave character. Its electric field component can 
be represented by $[51,53]$

$$
\underline{E}(\tau, \underline{r}, t)=\frac{1}{2} e^{-i \omega_{l}^{c}} \underline{u_{\eta}}(\underline{r})+c \cdot c .,
$$

where $\underline{r}, t$ stands for space and time coordinates. The complex frequency $\omega_{l}^{c}$ equals

$$
\omega_{l}^{c}=\omega_{l}-i \lambda_{l}
$$

where $\omega_{l}$ and $\lambda_{l}$ are the real frequency and decay constant of the $Z_{\text {th }}$ free oscillation $\left(\omega_{l}>0, \lambda_{l}>0\right)$. The functions $\underline{u}_{l}(\underline{r})$ are called natural-modes. They satisfy

$$
\underline{\nabla} \times \underline{\nabla} \times \underline{u}_{\eta}(\underline{r})=\frac{\left(\omega_{l}^{c}\right)^{2}}{e^{2}} \underline{u}_{l}(\underline{r}) \quad\left(\underline{r} \notin M_{1}, \ldots, M_{m}\right),
$$

where $c$ is the velocity of light in free space. For $|\underline{r}|+\infty$, the naturalmode $\underline{u}_{\imath}$ satisfies [51]

$$
\lim _{|\underline{r}| \rightarrow \infty} \underline{u}_{l}(\underline{r})=\underline{g}_{l}(\psi, \phi) \frac{e^{i \frac{\omega_{l}^{c}}{c}|\underline{r}|}}{|\underline{r}|},
$$

where $|\underline{r}|, \psi, \phi$ are spherical coordinates and $\underline{g}_{l}(\psi, \phi)$ is a single-valued vector function on the unit sphere and is tangential to it. It follows from (2-4) that each $\underline{u}_{l}$ increases exponentially for $|\underline{r}|+\infty$.

Concerning numerical calculations of natural-modes in actual configurations we first mention the work of Fox \& $\mathrm{Li}$ [52], in which an intuitive use is made of a reproduction principle. Calculations based on a strictly mathematical formulation of the electromagnetic boundary condition problem have been performed by Blok [53]. In a simple configuration, for instance a Fabry-Pérot interferometer, the free oscillations correspond, roughly speaking, to those field distributions which reproduce themselves, apart from a constant, after each round trip of the propagating electromagnetic field. A clear visualization of this reproduction principle for the determination of the modes belonging to a plane, anisotropic Fabry-Pérot 
interferometer has been given by De Lang [36]. A review of the theory of open resonators and the calculation of modes is given by Ronchi [54].

In the following we will be concerned with the determination of the "free oscillations" which result if somewhere in between the mirrors of the above-described resonator extra material is added. In doing so, we formally restrict ourselves to materials for which the dielectric properties can be entirely described by means of local constitutive equations. As it is our purpose to describe the electromagnetic field in a laser, we actually have in mind active materials, i.e., materials showing stimulated emission in some frequency region.

The contrast of the extra material with free space will be assumed to be so small that the natural-modes of the empty system can be used as "modes" of the complete system, at least for a number of applications. However, there are exceptional cases in which the very existence of the extra material leads to the occurrence of new modes. In fact, it has experimentally been shown by De Lang [36] that if a Fabry-Pérot type of resonator happens to possess an anisotropy matrix with two coinciding eigenstates, the presence of a laser medium can remove this type of degeneracy, which leads to the occurrence of a new mode. In that case it will be essential to extend the set of natural-modes with additional functions in order to yield a complete representation of the laser field. In general, such cases can be dealt with by starting with a conveniently adjusted "empty" resonator system, such that the corresponding new set of naturalmodes contains both the old ones and the additional ones. This will be discussed in more detail in Section 2.3 for the above mentioned Fabry-Pérot system.

The electromagnetic field within the resonator will be written as a linear combination of the $\underline{u}_{l}(\underline{r})$, while the time dependence of each expansion coefficient will be determined by substituting the field expression in the electromagnetic field equations, in which constitutive relations account for the presence of active material. In this point of view, a laser can thus only operate in the special field configurations corresponding to the natural-modes of the empty resonator. It should be realized that, in the presence of active material, one or more of the natural-modes can be excited during times exceeding $1 / \lambda_{l}$ by at least one order of magnitude. 
As is intuitively clear, the survival of such a mode depends on the balance between its decay rate $\lambda_{l}$ and the growth rate (gain) due to the active material. If necessary, the method can be extended in order to calculate the spatial form of the laser oscillations more accurately, but for our applications (we wish to determine the polarization behaviour of modes in a gas laser) the above-outlined method is sufficiently accurate.

In section 2.2 we will derive a rather general laser equation describing the time development of an excited laser mode (natural-mode) under the influence of the added (active) material. In Section 2.3 this equation will be applied to a Fabry-Pérot type of resonator with anisotropic mirrors. It is shown how we should deal in such a case with the evidently vectorial character of the natural-modes. It will follow that if the mirror anisotropy is sufficiently small, the natural-modes can always be combined in pairs so as to yield scalar mode patterns with slowly time-varying vectorial amplitudes, confirming the commonly adopted approach followed in References [36, 38, 39, 40].

Since all applications that will be dealt with in this study involve Fabry-Perot type of resonators, we will introduce in Section 2.4 parameters that clearly indicate the state of polarization of each laser mode. In Section 2.5, formal equations for the time evolution of these parameters will be derived. In these equations, coefficients occur that are to be determined from a detailed form of the electric polarization response of the active medium to the electromagnetic field. Such a form will be obtained in Chapter 3 for a special case (no magnetic field, $1 \rightarrow 0$ transition) and in Chapter 5 for a more general case.

\section{2 the general laser equation}

Let $\mathrm{V}_{\mathrm{A}}$ be a conveniently chosen finite volume containing all regions of active material but not $M_{1}, \ldots, M_{m}$ (see Figure 2.2). For $\underline{r} \in \mathrm{V}_{\mathrm{A}}$ the electric field $\underline{E}=\underline{E}(\underline{r}, t)$ is written as a linear combination of naturalmodes $\underline{u}_{\imath}(\underline{r})$ with time-dependent coefficients, or 


$$
\underline{E}(\underline{r}, t)=\frac{1}{2} \sum_{Z} E(Z, t) e^{-i \omega_{\eta} t} \underline{u}_{Z}(\underline{r})+c \cdot c,
$$

where $\underline{u}_{l}(\underline{r})$ satisfies $(2-3)$. Note that $\omega_{\eta}$ is the real part of $\omega_{\eta}^{c}$.

The electric field E satisfies the vector wave equation with a polarization term in the right-hand side which accounts for the presence of material,

$$
c^{2} \underline{\nabla} \times \underline{\nabla} \times \underline{E}+\partial_{t}^{2} \underline{E}=-\frac{1}{\varepsilon_{0}} \partial_{t}^{2} \underline{P},
$$

where $\underline{P}=\underline{P}(\underline{r}, t)$ is the induced dipole-moment density. In the case $\underline{P}=0$, i.e. in the absence of extra material, the general solution of (2-6) is characterized by exponentially decaying mode amplitudes $E(\mathcal{Z}, t)$ with decay constants $\lambda_{\eta}$. In order to achieve laser operation in a natural-mode, a dipole moment density $\underline{\mathrm{P}}$ has to be induced by the electric field such that it can at least compensate the loss constant $\lambda_{\ell}$.

The equations of motion for the $E(Z, t)$ in (2-5) will be derived by substitution of (2-5) in (2-6) and by projection of both sides of (2-6) on the $\underline{u}_{\imath}(\underline{r})$. It should be realized in this respect that in general the $\underline{u}_{2}$ are not orthogonal on $V_{A}$, that is

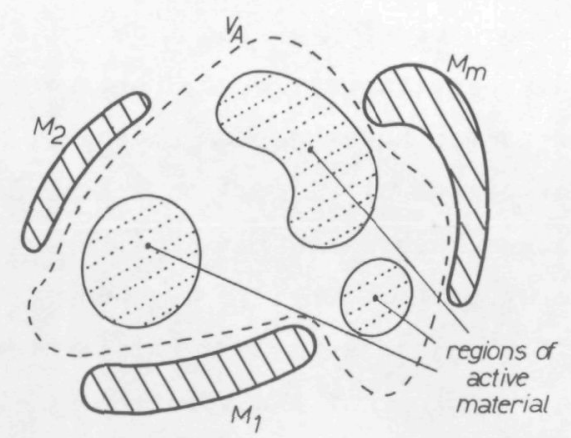

Fig. 2.2 Open resonator structure with regions of active medium. $\mathrm{V}_{\mathrm{A}}$ is a conveniently chosen volume containing all regions of active medium but not $\mathrm{M}_{1}, \ldots, \mathrm{M}_{m}$ 


$$
\int_{\mathrm{V}_{\mathrm{A}}} \mathrm{d} \underline{r} \underline{u}_{\tau}^{*}(\underline{r}) \cdot \underline{u}_{\tau},(\underline{r}) \neq 0 \text { for } Z \neq \tau^{\prime} \text {. }
$$

For that reason we have to apply a skew projection procedure. Denoting the projection coefficients of $\partial_{t}^{2} \underline{P}$ on $\underline{u}_{\tau}$ by

$$
\left.\partial_{t}^{2}\right|_{\underline{u}_{2}}=-\omega_{z}^{2} P(z, t) e^{-i \omega_{z} t}
$$

we easily obtain, using (2-3),

$$
\left(a_{t}-\lambda_{l}-2 i \omega_{l}\right)\left(a_{t}+\lambda_{l}\right) E(l, t)=\frac{\omega_{l}^{2}}{\varepsilon_{0}} F(l, t) .
$$

It should be remarked that, if the active medium gives rise to substantial contributions to $\underline{P}$ that lie completely outside the function space spanned by the $u_{\eta}(\underline{r})$, the set of equations (2-9) might give an incomplete description of laser operation. In fact, this is precisely what happens if the presence of active material leads to the occurence of new modes. We will, however, assume here that a representation of $\partial_{t}^{2} \underline{p}$ in terms of the naturalmodes is sufficiently complete in the above sense.

Equation (2-9) describes the time-evolution of the mode amplitudes. Since in (2-5) and (2-8) the rapidly oscillating terms, corresponding to the frequencies $\omega_{l}$ of the natural-modes, have been taken apart, we may expect that (2-9) will describe variations of $E(\tau, t)$ on a time scale much larger than $\omega_{l}^{-1}$. Hence, a weak-signal approximation [23], in which the second-order derivative and the terms proportional to $\lambda_{l}^{2}$ and $\lambda_{l} d_{t}$ in (2-9) are neglected, would be justified for modes having a sufficiently small $\lambda_{l}$. In this respect, it is instructive to note that the solution of (2-9) which we are seeking is also described by the first-order differential equation

$$
\left(\mathrm{a}_{t}+\lambda_{l}\right) E(\tau, t)=-\frac{\omega_{z}}{2 i \varepsilon_{0}} \sum_{n=0}^{\infty}\left(2 i \omega_{l}\right)^{-n}\left(\mathrm{a}_{t}-\lambda_{\tau}\right)^{n} P(\tau, t),
$$

at least if the series in the right-hand side is converging. In fact, 
(2-10) can be derived from (2-9) by performing an iteration procedure for $\left(d_{t}+\lambda_{l}\right) E(l, t)$, while it is easily verified by differentiation of $(2-10)$ with respect to time and, after that, by rearrangement of some terms in the right-hand side, that any solution of (2-10) is also a solution of (2-9).

Equation (2-10) allows for a straightforward simplification. The series expansion has terms proportional to $\omega_{\tau}^{-n}$, while the $P(\tau, t)$, just as the $E(\tau, t)$ according to the above discussion, are only weakly time dependent. Therefore, in all applications involving optical frequencies $\omega_{\ell}$ and smal1 decay constants $\lambda_{l} \ll \omega_{l}$, the terms with $n \geq 1$ will be small, and an excellent approximation will be obtained by neglecting all these terms. So, in this approximation, which is of course equivalent to the weak-signal approximation in Lamb's theory, we obtain

$$
\left(\mathrm{d}_{t}+\lambda_{\tau}\right) E(\tau, t)=\frac{i \omega_{\eta}}{2 \varepsilon_{0}} P(\tau, t) .
$$

Equation (2-11) can be considered as the general laser equation describing the time evolution of the weakly time-dependent mode amplitudes.

It is interesting to realize that, in the framework of the above projection procedure, the effect of different active regions in $V_{A}$ leads to additive contributions in the right-hand side of (2-11). This implies that, although each active region gives its own specific contribution to the time evolution of a mode amplitude, the right-hand side of (2-11) contains in fact a properly weighted sum of these contributions. In the case of natural-modes that are spatially uniform within the resonator, the weight factors appear to be equal to the respective volume fractions of the resonator. We will see in Chapter 7 that this additivity principle is crucial in understanding the polarization behaviour of a laser that is partly subject to a (transverse) magnetic field.

\section{3 the Zaser equation for a Fabry-Perrot type of resonator with anisotropic Zosses}

It is known from numerical studies that in many practical cases the natural-modes correspond to plane waves in a good approximation [52,53]. 


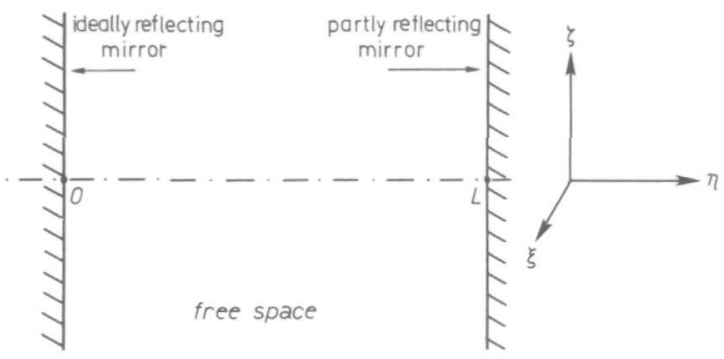

Fig.2.3 One-dimensional model for a Fabry-Pérot resonator

For such cases it seems therefore reasonable to use these plane waves as a representation of the free oscillations inside the open resonator. In this respect it is fortunate that a simple model for an open resonator, which is adequate for our purpose of describing longitudinal mode behaviour in lasers, exists. In this model the natural-modes defined in Section 2.1 can be determined analytically and exhibit the above-mentioned plane-wave character. The possible occurrence of anisotropic reflection properties of the mirrors in this resonator model can and will be included in a consistent manner.

The model is essentially a one-dimensional model, in which the field quantities are only functions of a single space variable. Let $(\xi, \eta, \zeta)$ be a rectangular, right-handed coordinate system. In the plane $\eta=0$ we assume the presence of an ideal, isotropically reflecting mirror, while the other mirror, placed in $\eta=L>0$, may have anisotropic reflection and transmission characteristics (see Figure 2.3). For the boundary condition in $n=0$ we simply state that the field vanishes identically there, while the boundary condition on the partly reflecting, anisotropic mirror will be formulated in terms of its reflection matrix: Let $\frac{1}{2} A(n) \exp (-i \omega t)+c \cdot c$. be the electric field component of an electromagnetic wave at frequency $\omega$ ( $\omega$ may be complex) and propagating in the $n$-direction towards $L$, and let $\frac{1}{2} \underline{B}(\eta) \exp (-i \omega t)+c . c$. be its reflected field. Note that $\underline{A}(\eta)$ and $\underline{B}(n)$ are vectors lying in the $(\xi, \zeta)$ plane. Then, excluding nonlinearities in the reflection characteristics, the reflection matrix $\underset{\underline{S}}{S}(\omega)$ can be defined as 


$$
\underline{B}(L)=-\underline{S}(\omega) \underline{A}(L) .
$$

Incidentally, the values of the four matrix elements of $\underline{S}$ can in principle be determined by analyzing the reflected field for various incident fields.

The analysis of the model is elementary and is given in Appendix A. The results can be summarized as follows: For $0<n<L$ the natural-modes are given by

$$
\begin{aligned}
\underline{u}_{n, q}(n)=\frac{1}{2 i}\left\{e^{\frac{1}{c}\left(\lambda_{n, q}-i \phi_{n, q}+i n \pi c / L\right) n}\right. \\
\\
\left.-e^{-\frac{1}{c}\left(\lambda_{n}, q-i \phi_{n, q}+i n \pi c / L\right) n}\right\}_{n, q},
\end{aligned}
$$

where: $n$ is the longitudinal mode number;

$q=1,2$ labels the two eigenvectors $\sigma_{n, 1}$ and $\underline{\sigma}_{n, 2}$ of $\underline{\underline{S}}$, corresponding to one longitudinal mode;

$\lambda_{n, q}$ is the decay constant associated with the mode $(n, q)$;

$\phi_{n, q}$ is the frequency shift of the mode $(n, q)$ with respect to $n \pi c / L$.

The eigenvectors $\underline{\sigma}_{n, q}$ are assumed to be normalized such that $\left|\sigma_{n, q}\right|=1$. They satisfy

$$
\stackrel{S}{=}\left(\omega_{n, q}^{c}\right)=e^{-\frac{2 L}{c}\left(\lambda_{n, q}-i \phi_{n, q}\right)} \stackrel{\sigma}{n, q}, \quad \text { Zie erratum }
$$

where the complex eigenfrequencies $\omega_{n, q}^{c}$ are given by

$$
\omega_{n, q}^{c}=n \pi c / L-\phi_{n, q}-i \lambda_{n, q} .
$$

For the model considered, the laser equation (2-11) reads

$$
\left(\mathrm{d}_{t}+\lambda_{n, q}\right) E(n, q, t)=\frac{i \omega_{n}}{2 \varepsilon_{0}} P(n, q, t),
$$


where we have taken

$$
\omega_{n}=n \pi c / L,
$$

i.e., the frequency shifts $\phi_{n, 1}$ and $\phi_{n, 2}$ in $\omega_{n, q}=n \pi c / L-\phi_{n, q}$ are neglected in the prefactor of $P(n, q, t)$. In accordance with (2.5), a general laser field within the Fabry-Pérot resonator will be represented by

$$
\underline{E}(n, t)=\frac{1}{2} \sum_{n, q} E(n, q, t) e^{-i \omega_{n}, q^{t}} \underline{u}_{n, q}(n)+c \cdot c .
$$

From now we will restrict ourselves to those cases in which the differences in both the amplitude loss and phase loss corresponding to one longitudinal mode number $n$ is so small that

$$
\frac{L}{c}\left|\lambda_{n, 1}-\lambda_{n, 2}\right| \ll 1 \text { and } \frac{L}{c}\left|\phi_{n, 1}-\phi_{n, 2}\right| \ll 1
$$

According to the representative numerical example given at the end of Appendix A, it can easily be verified that (2-19) is always satisfied for the usual mirrors applied in gas lasers. It is realized, however, that at least one of the conditions (2-19) may be violated if additional strongly polarizing components (for instance, a quarter wave length plate) are used within the resonator. Assuming (2-19) to be satisfied, we can replace (2-13) in a good approximation by

$$
\underline{u}_{n, q}(n)=u_{n}(n) \underline{\sigma}_{n, q}
$$

where

$$
u_{n}(n)=\frac{1}{2 i}\left\{e^{\frac{1}{c}\left(\lambda_{n}-i \phi_{n}+i n \pi c / L\right) n}-e^{-\frac{1}{c}\left(\lambda_{n}-i \phi_{n}+i n \pi c / L\right) \eta}\right\},(2-21)
$$

and where

$$
\lambda_{n}=\frac{1}{2}\left(\lambda_{n, 1}+\lambda_{n, 2}\right) ; \quad \phi_{n}=\frac{1}{2}\left(\phi_{n, 1}+\phi_{n, 2}\right) .
$$


By using (2-20), we can rewrite the expression (2-18) as

$$
\underline{E}(n, t)=\frac{1}{2} \sum_{n} \underline{E}(n, t) e^{-i \omega t t} u_{n}(n)+c \cdot c .,
$$

where

$$
E(n, t)=\sum_{q=1}^{2} E(n, q, t) e^{i \phi_{n, q} t} \stackrel{\sigma}{n, q}^{t} .
$$

If we exclude for the moment that $\sigma_{n, 1}$ and $\underline{\sigma}_{n, 2}$ are coinciding, then the thus introduced vector amplitude $\underline{E}(n, t)$ can in principle describe any (weakly time-dependent) vector state. This property will allow us in the next section to assign to each longitudinal mode combination a weakly timedependent state of polarization in agreement with usual descriptions of mode-polarization behaviour. The case in which $\underline{\sigma}_{n, 1}$ and $\underline{\sigma}_{n, 2}$ are equal, or at least equal up to a complex prefactor of absolute value one, will be treated further on.

It is convenient to write the projected parts of the polarization in a similar way

$$
\underline{P}(n, t)=\sum_{q=1}^{2} P(n, q, t) e^{i \phi_{n}, q^{t}} \underline{\sigma}_{n, q} .
$$

If we then start from (2-24), it is easy to derive an equation for $E(n, t)$. Using (2-16) and (2-25), we obtain

$$
\mathrm{d}_{t} \underline{E}(n, t)+\frac{1}{2} \underline{\Gamma} \underline{\underline{\Gamma}} \underline{E}(n, t)=\frac{i \omega_{n}}{2 \varepsilon_{0}} \underline{P}(n, t),
$$

where the loss tensor $\Gamma_{n}$ is defined by

$$
\stackrel{\Gamma}{=}_{n} \stackrel{\sigma}{n, q}_{n}=2\left(\lambda_{n, q}-i \phi_{n, q}\right) \underline{\sigma}_{n, q} \quad(q=1,2) .
$$

The factor $\frac{1}{2}$ in front of $\Gamma_{n}$ in the left-hand side of (2-26) has been added to obtain agreement with current notations [23, 39, 40]. Equation $(2-26)$ is the vector laser equation for a Fabry-Pérot type of resonator. In the usual treatments [39, 40] the loss term is added in a phenomenological way. It can be verified that this equation can be derived by the same procedure if two 
strongly reflecting, weakly anisotropic mirrors are considered in the onedimensional Fabry-Pérot resonator model. In that case, $I_{n}$ measures the (anisotropic) loss of the nth longitudinal mode combination due to the combined effect of the two mirrors.

Let us now consider the exceptional case in which at least for one longitudinal mode the two polarization vectors $\sigma_{n, 1}$ and $\sigma_{n, 2}$ coincide. It has been shown by De Lang [36] that this kind of degenerate anisotropy can indeed occur in practice; it even played an essential role in his experimental study of laser operation. De Lang observed that, although the empty resonator was prepared so as to possess two coinciding right-handed circularly polarized eigenstates, the laser operated in an almost linearly polarized state of polarization. From this he concluded that the nonlinear polarization response of the active medium introduced an additional anisotropy such that the original degeneracy was removed. In our description of laser operation this means that, in case of such a degenerate anisotropy, the set of natural-modes is too small, since the polarization response contains substantial contributions that will not be accounted for after projection. A straightforward way of enlarging the set of natural-modes is by starting with an empty resonator system in which the degeneracy is removed by the formal addition of a sma11, suitably chosen, extra anisotropy. The above-given procedure can then be followed in order to derive the laser equation (2-26). After that, the correct $\Gamma_{n}$ tensor can be obtained by letting the additional anisotropy approach zero.

It follows from (2-14) and (2-27) that in the case of a large mirror reflectivity, the loss matrix is approximately given by

$$
\underline{\Gamma}_{n} \simeq \frac{c}{L}\left\{\underline{\underline{1}}-\underline{\underline{S}}\left(\omega_{n}^{c}\right)\right\},
$$

where $\omega_{n}^{c}=n \pi c / L-\phi_{n}-i \lambda_{n}$, This confirms the intuitive point of view in which the contribution of the mirrors to the time evolution of the mode amplitude can be thought of as being smoothed out in time during one pass of the field through the resonator.

As stated at the end of Section $2.2, \underline{P}(n, t)$ can be subdivided into contributions from individual layers of active medium. This, together with the above-derived resonator loss term, leads to the following general 
equations for the time evolution of the respective mode amplitudes:

$$
\mathrm{d}_{t} \underline{E}(n, t)=\left.\partial_{t} \underline{E}(n, t)\right|_{\text {resonator }}+\left.\sum_{\substack{\text { all } \\ \text { layers }}} \partial_{t} \underline{E}(n, t)\right|_{\text {layer }},
$$

where

$$
\begin{aligned}
& \left.\partial_{t} E(n, t)\right|_{\text {resonator }}=-\frac{1}{2} \stackrel{\Gamma}{=} \underline{E}(n, t), \\
& \left.\partial_{t} \underline{E}(n, t)\right|_{\text {layer }}=\left.\frac{i \omega_{n}}{2 \varepsilon_{0}} \underline{P}(n, t)\right|_{\text {layer }},
\end{aligned}
$$

and $\left.\underline{P}(n, t)\right|_{\text {layer }}$ is the contribution to $\underline{P}(n, t)$ from the layer considered.

\section{4 introduction of mode-polarization parometers}

As the main object of the present study is to investigate the polarizing behaviour of a gas laser due to various mechanisms, such as the active medium, the mirrors and externally applied magnetic fields, it is worthwile to introduce quantities that indicate the state of the electromagnetic field of a mode in a clear way, and in particular its state of polarization. Depending on the type of phenomenon to be described, we will use two different sets of polarization parameters. Though both descriptions are of course equivalent, it turns out that equations adopt easier forms by making the proper choice of parameter set.

The first set of parameters to be used is given by

$$
\begin{array}{ll}
I_{n}(t)=|\underline{E}(n, t)|^{2}, & \left(I_{n} \geq 0\right) ; \\
R_{n}(t)=\arctan \left\{\left|E_{\xi}(n, t)\right| /\left|E_{\zeta}(n, t)\right|\right\} & , \quad\left(0 \leq R_{n} \leq \pi / 2\right) ; \\
\theta_{n}(t)=\arg \left\{E_{\xi}(n, t) E_{\zeta}(n, t)^{*}\right\}, & \left(-\pi<\theta_{n} \leq \pi\right) .
\end{array}
$$

Here, $E_{\xi}(n, t)$ and $E_{\zeta}(n, t)$ indicate the $\xi$ - and $\zeta$-components of the field vector $E(n, t)$. The parameter $I_{n}$ is a measure for the intensity of the $n$th mode; $R_{n}$ is an angle related to the ratio of the amplitude components 
along the $\xi$ - and $\zeta$-axis, respectively, and $\theta_{n}$ is their phase difference. The fourth independent quantity that, strictly speaking, has to be defined in order to fix the two-component complex vector $E(n, t)$ completely, is the total phase angle arg $\left\{E_{\xi}(n, t) E_{\zeta}(n, t)\right\}$, measuring the small deviation of the operation frequency from $n \pi c / L$. The influence of this quantity in the polarization behaviour of a laser will however be disregarded,

The state of polarization of the $n$th mode is completely determined by specifying $R_{n}$ and $\theta_{n}$. For instance $\theta_{n}=0$ means linear polarization, while the plane of polarization forms an angle $R_{n}$ with the positive $\zeta$-axis. Similarly, $\theta_{n}=\pi$ is also linear polarization, but now the polarization plane forms an angle $-R_{n}$ with the positive $\zeta$-axis. The case $R_{n}=\pi / 4$ and $\Theta_{n}=\pi / 2(-\pi / 2)$ corresponds to right (left)-handed circular polarization.

The second set of parameters consists, apart from $I_{n}[$ see (2-32)], of the ellipticity $x_{n}$ and the azimuth $u_{n}$, which are more directly related to the polarization ellipse of the mode. The ratio of short to long axis of the ellipse is given by $\tan \left|x_{n}\right|\left(-\pi / 4 \leq x_{n} \leq \pi / 4\right)$. The sense of rotation is right-handed if $x_{n}>0$ and left-handed if $x_{n}<0$. The azimuth $u_{n}$ is defined as the angle between the long axis and the $\zeta$-axis $\left(-\pi / 2<u_{n} \leq \pi / 2\right)$. When the mode index $n$ is omitted, the correspondence with $R_{n}$ and $\theta_{n}$ is as follows:

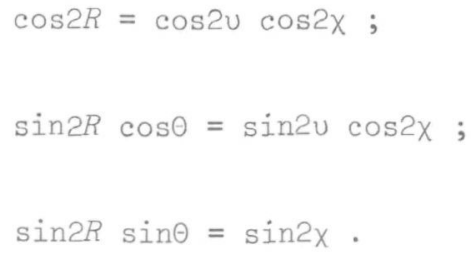

In Fig. 2.4 the meaning of the different polarization parameters has been visualized for a special case of elliptic polarization. In this example the sense of rotation is right-handed. 


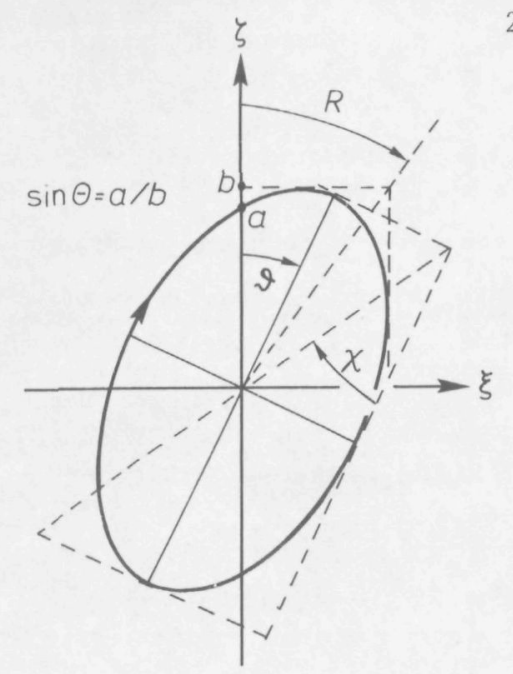

Fig. 2.4 Polarization ellipse with polarization parameters $x$ and $u$. The alternative parameters $R$ and $\theta$ have been indicated as we11.

\section{5 equations for the polarization parameters (single-frequency case)}

In order to transform (2-26) into a set of first-order differential equations for the polarization parameters introduced in Section 2.4, one should like to have an explicit expression for the induced dipole-moment density in the active medium, in terms of the driving-field vectors $\underline{E}(n, t)$. In the next chapter we shall derive just such an explicit expression for the case of a special spectral transition, in the absence of externally applied fields, and we will restrict ourselves to the case in which only one single vectorial mode is excited. In Chapter 5 the more general case will be treated, involving all types of transitions, externally applied magnetic fields and multi-mode electric fields.

For the relatively simple case of single-mode operation it is however easy to write down quite generally a formal expression for the polarization response as a power series in the driving electric field amplitudes, without specifying, however, the coefficients. We will derive such an expression for the case in which a homogeneous layer of medium is subject to a uniform magnetic field $\underline{H}$. It turns out to be helpful to introduce a new matum matum right-handed coordinate system $(x, y, z)$, such that the $z$-axis is along $\underline{H}$ 2rom orram 
Let us suppose that in the medium a single-frequency electric field is present, given by

$$
\underline{E}(\underline{r}, t)=\frac{1}{2}\left\{E_{1} \frac{\underline{\hat{x}}-i \hat{\underline{y}}}{\sqrt{2}}+E_{-1} \frac{\underline{\hat{x}}+i \underline{\hat{y}}}{\sqrt{2}}+E_{0} \underline{\hat{z}}\right\} e^{-i \omega t} u(\underline{r})+c . c .,
$$

where $\underline{\hat{x}}, \underline{\hat{y}}$ and $\underline{\hat{z}}$ are unit vectors along the $x-, y$-, and $z$-axis, respective$1 y$. The projected part of the polarization response at frequency $\omega$ can be represented similarly:

$$
\underline{P}^{\operatorname{proj}}(\underline{r}, t)=\frac{1}{2}\left\{P_{1} \frac{\underline{\hat{x}}-i \underline{\hat{y}}}{\sqrt{2}}+P_{-1} \frac{\underline{\hat{x}}+i \underline{\hat{y}}}{\sqrt{2}}+P_{0} \underline{\hat{z}}\right\} e^{-i \omega t} u(\underline{r})+c \cdot c .
$$

Until further notice, the quantities $P_{1}, P_{-1}$. and $P_{0}$ will remain unknown. However, the formal relationship between the $P_{l}$ and $E_{l}$ is as follows (omitting 5 th and higher-order terms in the driving field):

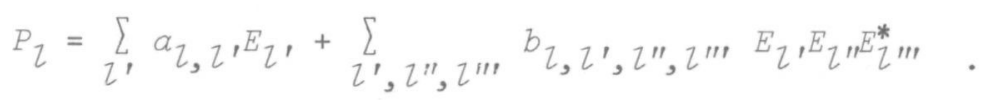

In (2-38) no even-order terms are present, since such terms do not refer to the proper frequency $\omega$. Equation $(2-38)$ can be reduced by considering transformation properties under a rotation of the coordinate system over an angle $\psi$ around the $z$-axis. Assuming an isotropic medium, we then have

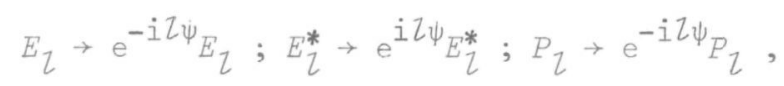

so that in the first summation in (2-38) only the term with $\tau^{\prime}=\tau$ survives, while in the second summation $\eta^{\prime}+\tau^{\prime \prime}-\tau^{\prime \prime \prime}=\tau$. Hence, (2-38) can formally be written

$$
\begin{gathered}
\left(\omega / \varepsilon_{0}\right) P_{1}=-a_{1} E_{1}+b_{1,1} E_{1}\left|E_{1}\right|^{2}+b_{1,1} E_{1}\left|E_{0}\right|^{2}+b_{1,-1} E_{1}\left|E_{-1}\right|^{2}+c_{1} E_{-1}^{*} E_{0}^{2} ; \\
\left(\omega / \varepsilon_{0}\right) P_{0}=-a_{0} E_{0}+b_{0,1} E_{0}\left|E_{1}\right|^{2}+b_{0,0} E_{0}\left|E_{0}\right|^{2}+b_{0,-1} E_{0}\left|E_{-1}\right|^{2}+c_{0} E_{0}^{*} E_{1} E_{-1} \\
\left(\omega / \varepsilon_{0}\right) P_{-1}=-a_{-1} E_{-1}+b_{-1,1} E_{-1}\left|E_{1}\right|^{2}+b_{-1,0} E_{-1}\left|E_{0}\right|^{2}+b_{-1,-1} E_{-1}\left|E_{-1}\right| 2 \\
+c_{-1} E_{1}^{*} E_{0}^{2},
\end{gathered}
$$


where a new set of coefficients has been introduced and where the factor $\omega / \varepsilon_{0}$ in the left-hand sides has been added for convenience. The explicit form of the $a, b$ and $c$ coefficients should follow from an analysis of the interaction between the electric field and the atoms, as will be carried out in Chapters 3 and 5. The coefficients will be functions of, among others, the field f'requency $\omega$ and the magnitude $H=|\underline{H}|$ of the magnetic field.

We conclude this chapter by focussing on two important cases, namely (a) the case of an axial magnetic field, i.e., the direction of propagation of the electric field coincides with the direction of $\underline{H}$ and (b) the case of a transverse magnetic field, i.e., the propagation vector is perpendicular to $\underline{\mathrm{H}}$.

(a) axial magnetic field. The electric-field vector has only $x-$ and $y$ components. Taking $E_{0}=0$ in $(2-40)-(2-42)$, we obtain

$$
\begin{aligned}
& \left(\omega / \varepsilon_{0}\right) P_{1}=-a_{1} E_{1}+b_{1,1} E_{1}\left|E_{1}\right|^{2}+b_{1,-1} E_{1}\left|E_{-1}\right|^{2}, \\
& \left(\omega / \varepsilon_{0}\right) P_{-1}=-a_{-1} E_{-1}+b_{-1,1} E_{-1}\left|E_{1}\right|^{2}+b_{-1,-1} E_{-1}\left|E_{-1}\right|^{2} .
\end{aligned}
$$

The axial symmetry favours a representation of the state of polarization in terms of the ellipticity $x$ and the azimuth $u$. With (2-43) and (2-44) and the relationships [which can be deduced from $(2-32)-(2-35)]$ :

$$
\begin{aligned}
& I=\left|E_{-1}\right|^{2}+\left|E_{1}\right|^{2} ; \\
& \sin 2 x=I^{-1}\left(\left|E_{-1}\right|^{2}-\left|E_{1}\right|^{2}\right) ; \\
& u=\frac{1}{2} \arg \left(E_{1} E_{-1}^{*}\right)
\end{aligned}
$$

it is easy to derive equations for the time development of these parameters. Starting from (2-26), we find 


$$
\begin{aligned}
& \mathrm{d}_{t} \mathrm{x}=\left.\partial_{t} \mathrm{x}\right|_{\text {resonator }}+\left.\sum_{\substack{\text { aly } \\
\text { layers }}} \partial_{t} \mathrm{x}\right|_{\text {layer }} \\
& \cos 2 x \mathrm{a}_{t} u=\left.\cos 2 x \partial_{t} u\right|_{\text {resonator }}+\left.\sum_{\substack{\text { aly } \\
\text { layers }}} \cos 2 x \partial_{t} u\right|_{\text {layer }} \\
& a_{t} I=\left.\partial_{t} I\right|_{\text {resonator }}+\left.\underset{\substack{\text { ajII } \\
\text { layers }}}{ } \partial_{t} I\right|_{\text {layer }}
\end{aligned}
$$

It is noted that the additivity is not destroyed when a tranformation to the polarization parameters is made. The resonator-induced contributions in $(2-48)$ - (2-50) have been evaluated in Appendix B for the case of a loss tensor that is as general as possible. The contributions due to a layer of medium subject to an axial, uniform magnetic field are given by

$$
\begin{aligned}
& \left.2 \partial_{t} x\right|_{\text {layer }}=\cos 2 x\left\{a_{1}^{\prime \prime}+I\left(-b_{2}^{\prime \prime}+c^{\prime \prime} \sin 2 x\right)\right\} ; \\
& \left.2 \cos 2 x \partial_{t}^{u}\right|_{\text {layer }}=\cos 2 x\left\{a_{1}^{\prime}+I\left(-b_{2}^{\prime}+c^{\prime} \sin 2 x\right)\right\} ; \\
& \left.\left(I^{-1}\right) \partial_{t} I\right|_{\text {layer }}=a^{\prime \prime}+a_{1}^{\prime \prime} \sin 2 x-I\left(b^{\prime \prime}+b_{1}^{\prime \prime} \sin 2 x-c^{\prime \prime} \sin ^{2} 2 x\right)
\end{aligned}
$$

where primes are used to indicate real parts of quantities and double primes denote imaginary parts, and where the $a, b$ and $c$ coefficients are defined by

$$
\begin{aligned}
& a=\frac{1}{2}\left(a_{1}+a_{-1}\right) ; a_{1}=\frac{1}{2}\left(a_{-1}-a_{1}\right) ; \\
& b=\frac{1}{4}\left(b_{1,1}+b_{-1,-1}+b_{1,-1}+b_{-1,1}\right) ; \\
& b_{1}=\frac{1}{2}\left(b_{-1,-1}-b_{1,1}\right) ; \\
& b_{2}=\frac{1}{4}\left(b_{-1,-1}-b_{1,1}+b_{-1,1}-b_{1,-1}\right) ; \\
& c=\frac{1}{4}\left(b_{-1,1}+b_{1,-1}-b_{1,1}-b_{-1,-1}\right) .
\end{aligned}
$$

The indexed coefficients, i.e., $a_{1}, b_{1}$ and $b_{2}$, vanish for $H=0$. 
(b) transverse magnetic field, The propagation vector of the electric field is in this case perpendicular to $\underline{H}$. If we choose the $y$-axis in the propagation direction, the electric field has only $x$ - and $z$-components. It turns out to be less cumpersome to use the cartesian components of the field than the circular ones, or

$$
E_{z}=E_{0} ; E_{x}=\frac{1}{\sqrt{2}}\left(E_{1}+E_{-1}\right) ; E_{y}=-\frac{i}{\sqrt{2}}\left(E_{1}-E_{-1}\right)=0
$$

and

$$
P_{z}=P_{0} ; P_{x}=\frac{1}{\sqrt{2}}\left(P_{1}+P_{-1}\right) .
$$

Using (2-40) - (2-42) as well as $(2-55)$ and $(2-56)$, we derive

$$
\begin{aligned}
& \left(\omega / \varepsilon_{0}\right) P_{x}=\left(-a_{x}+b_{x x}\left|E_{x}\right|^{2}+b_{x z}\left|E_{z}\right|^{2}\right) E_{x}+c_{x} E_{z}^{2} E_{x}^{*} ; \\
& \left(\omega / \varepsilon_{0}\right) P_{z}=\left(-a_{z}+b_{z z}\left|E_{z}\right|^{2}+b_{z x}\left|E_{x}\right|^{2}\right) E_{z}+c_{z} E_{z}^{*} E_{x}^{2},
\end{aligned}
$$

where

$$
\begin{aligned}
& a_{x}=\frac{1}{2}\left(a_{1}+a_{-1}\right) ; a_{z}=a_{0} ; \\
& b_{x x}=\frac{1}{4}\left(b_{1,1}+b_{-1,-1}+b_{1,-1}+b_{-1,1}\right) ; \\
& b_{z z}=b_{0,0} ; \\
& b_{x z}=\frac{1}{2}\left(b_{1,0}+b_{-1,0}\right) ; b_{z x}=\frac{1}{2}\left(b_{0,1}+b_{0,-1}\right) ; \\
& c_{x}=\frac{1}{2}\left(c_{1}+c_{-1}\right) ; c_{z}=\frac{1}{2} c_{0} .
\end{aligned}
$$

It is more convenient in this case to represent the state of polarization in terms of $I, R$ and $\theta$. The time evolution of these parameters can again be written as a sum of contributions due to the empty resonator and due to medium layers. The resonator-induced contributions are given in Appendix B, while the contributions due to a layer of medium subject to a transverse, uniform magnetic field read: 


$$
\begin{aligned}
& \left.2 \partial_{t}\right|_{\text {medium }}=\begin{array}{c}
\sin 2 R\left\{-\alpha_{1}^{\prime \prime} / 2+I\left(-\gamma^{\prime \prime} \cos 2 \theta \cos 2 R+\gamma^{\prime} \sin 2 \theta \quad(2-60)\right.\right. \\
\left.\left.-\gamma_{1}^{\prime} \sin 2 \theta \cos 2 R+\gamma_{1}^{\prime \prime} \cos 2 \theta+\beta_{2}^{\prime \prime}+\delta^{\prime \prime} \cos 2 R\right)\right\} ;
\end{array}
\end{aligned}
$$

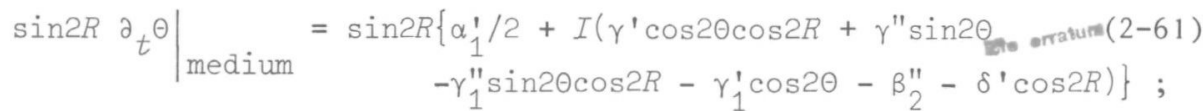

$$
\begin{aligned}
& \begin{array}{r}
\left.I^{-1} \partial_{t} I\right|_{\text {medium }}=\alpha^{\prime \prime}-\alpha_{1}^{\prime \prime} \sin ^{2} R-I\left\{\beta^{\prime \prime}-\beta_{1}^{\prime \prime} \sin ^{2} R\right. \\
\left.+\left(\gamma^{\prime \prime} \cos 2 \theta+\gamma_{1}^{\prime} \sin 2 \theta-\delta "\right) \sin ^{2} R \cos ^{2} R\right\},
\end{array}
\end{aligned}
$$

where we have used

$$
\begin{aligned}
& \alpha=a_{z} ; \beta=b_{z z} ; \gamma=c_{z}+c_{x} ; \\
& \delta=b_{z z}+b_{x x}-b_{x z}-b_{z x} ; \\
& \alpha_{1}=a_{z}-a_{x} ; \beta_{1}=b_{z z}-b_{x x} ; \\
& \beta_{2}=b_{z z}-b_{x x}+b_{z x}-b_{x z} ; \gamma_{1}=c_{z}-c_{x} .
\end{aligned}
$$

The indexed coefficients, i.e., $\alpha_{1}, \beta_{1}, \beta_{2}, \gamma_{1}$ vanish for $\underline{H}=0$, while $\gamma$ and $\delta$ coincide, as follows from symmetry considerations. 


\section{RESPONSE OF A GAS TO AN ELECTROMAGNETIC FIELD $(j=1 \rightarrow j=0$ TRANSITION CASE $)$}

In the preceding chapter a formal relationship between fields $\underline{E}$ and $\underline{P}$ in the resonator (i.e., a constitutive relation) has been derived by symmetry arguments. In order to obtain the explicit values of the coefficients occurring in this relation, we will study the response of a gas of identical atoms to an electromagnetic field in some detail. The purpose in the present chapter is to give a transparent treatment for a case in which the atomic level degeneracies are low. A more general treatment, involving all types of level degeneracies, multi-frequent electric fields and externally applied magnetic fields, will be given in Chapter 5.

We will study the case of a $j=1 \rightarrow j=0$ transition, where the respective $j$ values indicate the total-angular-momentum quantum numbers belonging to the two atomic energy levels involved in the interaction with the electromagnetic field, while the arrow points in the direction of the level of lowest energy. Interaction of the electromagnetic field with this two-level system will be considered in sufficient detail to enable us to determine the above-mentioned coefficients. It will be assumed that no externally applied magnetic fields are present. As an example of the transition we mention the $2 \mathrm{~s}_{2} \rightarrow 2 \mathrm{p}_{1}$ Ne transition corresponding with radiation at a wavelength of $1.52 \mu \mathrm{m}$.

As the $j=1$ level has threefold degeneracy and the $j=0$ level is nondegenerate, there are four atomic levels involved. This is also the case for $j=\frac{1}{2} \rightarrow j=\frac{1}{2}$ and $j=0 \rightarrow j=1$ transitions. However, 1 aser operation on a $\frac{1}{2} \rightarrow \frac{1}{2}$ transition has not been reported yet, while the case of a $0 \rightarrow 1$ transition can be easily obtained from the $1 \rightarrow 0$ case by making some trivial substitutions in the analysis of the latter. We prefer therefore to treat the $j=1 \rightarrow j=0$ transitions first. Theoretical treatments of this case have been given by Durand [30] and by Culshaw \& Kannelaud [31], while this case has also been incluaded in the more general articles by Van Haeringen [39] and by Sargent et al.[40,41]. However, it 
has been shown by Polder \& Van Haeringen in a later paper [27] that the effect of atomic collisions leads to a polarization response quite different from that derived in References $[30,31,39,40]$. In the present treatment these collision effects are extensively dealt with. They will be incorporated along the lines followed by Durand, though our conclusions will differ from his [30].

In Section 3.1 we will derive general equations of motion, i.e., first-order differential equations, describing the time evolution of expectation values belonging to atomic observables due to a perturbation of the electric-dipole kind. In Section 3.2 approximate solutions of the equations will be derived by setting up an iteration scheme in which the expectation values of the observables are expanded as a power series in the electric-field amplitude. The macroscopic polarization response of the atomic medium, i.e., the gas, will be calculated in Section 3.3.

\section{1 derivation of the equations of motion of expectation values}

In the case to be considered the constituents of the gas are identical atoms of which two energy levels are relevant in describing the response to an electric perturbation. The upper level a corresponds to a triplet state with total-angular-momentum quantum number $j_{\mathrm{a}}=1$ and energy $\hbar \Omega_{\mathrm{a}}$; the lower state $\mathrm{b}$ is a $j_{\mathrm{b}}=0$ singlet state with energy $\hbar \Omega_{\mathrm{b}}$. If $(x, y, z)$ is a right-handed cartesian coordinate system, the three independent wave functions belonging to the upper level are chosen to be the eigenfunctions of the $z$-component of the angular-momentum operator. They will be denoted by $\Psi_{m}^{\mathrm{a}}$, where $m$ is the magnetic quantum number, assuming the values $0, \pm 1$. The lower level wave function is denoted by $\psi^{\mathrm{b}}$. Our interest is in operators acting in the four-dimensional function space spanned by these four wave functions. As any operator can be represented by a $4 \times 4$ matrix with respect to the base

$$
\left\{\Psi_{1}^{\mathrm{a}}, \Psi_{0}^{\mathrm{a}}, \Psi_{-1}^{\mathrm{a}}, \Psi^{\mathrm{b}}\right\},
$$

precisely 16 linearly independent operators exist. It is convenient to 
choose a basic set of operators in accordance with the transformation properties under three-dimensional proper rotations. This leads to the set of scalar, vector and tensor operators given below.

There appear to be two operators transforming as scalars. They correspond to the global-population operators $N_{a}$ and $N_{b}$ of the upper and lower level, respectively. We choose their normalizations such that the matrix representations with respect to (3-1) are given by

$$
N_{a}=\frac{1}{3}\left(\begin{array}{llll}
1 & 0 & 0 & 0 \\
0 & 1 & 0 & 0 \\
0 & 0 & 1 & 0 \\
0 & 0 & 0 & 0
\end{array}\right) ; N_{b}=\left(\begin{array}{llll}
0 & 0 & 0 & 0 \\
0 & 0 & 0 & 0 \\
0 & 0 & 0 & 0 \\
0 & 0 & 0 & 1
\end{array}\right) .
$$

Nine operators can be combined in three sets of three operators, each set corresponding to a vector observable. The components of these operators with respect to the $(x, y, z)$ system are chosen as, respectively,

$$
\begin{aligned}
& J_{x}=\frac{1}{\sqrt{2}}\left(\begin{array}{llll}
0 & 1 & 0 & 0 \\
1 & 0 & 1 & 0 \\
0 & 1 & 0 & 0 \\
0 & 0 & 0 & 0
\end{array}\right) ; J_{y}=\frac{1}{\sqrt{2}}\left(\begin{array}{rrrr}
0 & -i & 0 & 0 \\
i & & -i & 0 \\
0 & i & 0 & 0 \\
0 & 0 & 0 & 0
\end{array}\right) ; J_{z}=\left(\begin{array}{rrrr}
1 & 0 & 0 & 0 \\
0 & 0 & 0 & 0 \\
0 & 0 & -1 & 0 \\
0 & 0 & 0 & 0
\end{array}\right) ; \\
& D_{x}=\frac{1}{\sqrt{2}}\left(\begin{array}{cccr}
0 & 0 & 0 & -1 \\
0 & 0 & 0 & 0 \\
0 & 0 & 0 & 1 \\
-1 & 0 & 1 & 0
\end{array}\right) ; D_{y}=\frac{1}{\sqrt{2}}\left(\begin{array}{cccc}
0 & 0 & 0 & i \\
0 & 0 & 0 & 0 \\
0 & 0 & 0 & i \\
-i & 0 & -i & 0
\end{array}\right) ; D_{z}=\left(\begin{array}{llll}
0 & 0 & 0 & 0 \\
0 & 0 & 0 & 1 \\
0 & 0 & 0 & 0 \\
0 & 1 & 0 & 0
\end{array}\right) ; \\
& \hat{D}_{x}=\frac{1}{\sqrt{2}}\left(\begin{array}{rrrr}
0 & 0 & 0 & -i \\
0 & 0 & 0 & 0 \\
0 & 0 & 0 & i \\
i & 0 & -i & 0
\end{array}\right) ; \hat{D}_{y}=\frac{1}{\sqrt{2}}\left(\begin{array}{cccc}
0 & 0 & 0 & -1 \\
0 & 0 & 0 & 0 \\
0 & 0 & 0 & -1 \\
-1 & 0 & -1 & 0
\end{array}\right) ; \hat{D}_{z}=\left(\begin{array}{llll}
0 & 0 & 0 & 0 \\
0 & 0 & 0 & i \\
0 & 0 & 0 & 0 \\
0 & -i & 0 & 0
\end{array}\right) ;
\end{aligned}
$$

Here, $\underline{J}$ is proportional to the angular-momentum operator; $\underline{D}$ is proportional to the electric-dipole operator and $\hat{\underline{D}}$ is proportional to the displacementcurrent operator. Finally, five remaining operators can be chosen so as to correspond to the components of the quadrupole-momentum tensor operator 2) i.e., 


$$
\begin{aligned}
& 2_{x y}=\left(\begin{array}{rrrr}
0 & 0 & -i & 0 \\
0 & 0 & 0 & 0 \\
i & 0 & 0 & 0 \\
0 & 0 & 0 & 0
\end{array}\right) ; \frac{1}{2}\left(R_{x x}-2_{y y}\right)=\left(\begin{array}{llll}
0 & 0 & 1 & 0 \\
0 & 0 & 0 & 0 \\
1 & 0 & 0 & 0 \\
0 & 0 & 0 & 0
\end{array}\right) ;
\end{aligned}
$$

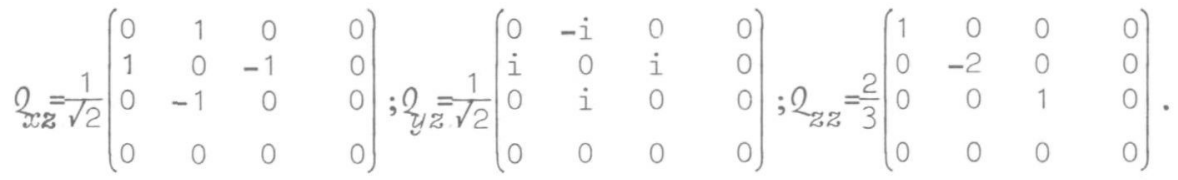

It is easily verified that the unperturbed hamiltonian $H_{0}$ can be written as

$$
H_{0}=3 \hbar \Omega_{a} N_{a}+\hbar \Omega_{b} N_{b} .
$$

It can be shown that all operators given in (3-2) - (3-6) can be generated from $\underline{D}$, by using the following identities:

$$
\begin{aligned}
& N_{\mathrm{a}}=(\underline{J} \cdot \underline{J}) / 3 ; \\
& N_{\mathrm{b}}=\left(\underline{D} \cdot \underline{D}-\frac{1}{2} \underline{J} \cdot \underline{J}\right) / 3 ; \\
& \underline{J}=-i \sqrt{ } 2 \underline{D} \times \underline{D} ; \\
& \underline{\hat{D}}=\frac{1}{2}(\underline{J} \times \underline{D}-\underline{D} \times \underline{J}) ; \\
& \underline{D}=-\underline{D} \otimes \underline{D}=\underline{J} \otimes \underline{J} .
\end{aligned}
$$

Here, the symbols "•" and "x" mean the usual scalar and vector product, respectively. The symbol " $\otimes "$ is used if two vectors are combined to yield a tensor of the second rank according to the rule

$$
(\underline{A} \otimes \underline{B})_{\xi \eta}=A_{\xi} B_{\eta}+A_{\eta} B_{\xi}-\frac{2}{3}(\underline{A} \cdot \underline{B}) \delta_{\xi \eta},
$$

where $\xi$ and $n$ are cartesian indices. Furthermore, in cases where $\underline{\underline{B}}$ is a tensor of rank two and $\underline{A}$ is a vector, the product $\underline{B A}$ is as usual

$$
(\underline{B A})_{\xi}=\sum_{\eta} B_{\xi \eta} A_{\eta}
$$


From $(3-4),(3-5)$ and $(3-7)$ we derive the useful identity

$$
\hat{D}=\frac{1}{i \hbar\left(\Omega_{a}-\Omega_{b}\right)}\left[\underline{D}, H_{\theta}\right],
$$

where $[$,$] stands for the commutator of two operators.$

Let us now investigate how a perturbing electric field $\underline{E}=\underline{E}(r, t)$ will influence observables. In the usual electric-dipole approximation, also adopted here, the electric field is taken to be constant over the dimensions of the atom. The field then couples to the electric-dipole operator $\underline{\mu}$, which is given by [62]

$$
\underline{\mu}=\sqrt{2} p \underline{0},
$$

where

$$
p=\frac{1}{\sqrt{2}}\left\langle\Psi_{0}^{\mathrm{a}}\left|\mu_{z}\right| \Psi^{\mathrm{b}}\right\rangle \text {, }
$$

and $\left\langle\Psi_{0}^{a}\left|\mu_{z}\right| \Psi^{b}\right\rangle$ is the matrix element of $\mu_{z}$ between the states with wave functions $\Psi_{0}^{\mathrm{a}}$ and $\Psi^{\mathrm{b}}$, respectively. The interaction hamiltonian is given by

$$
H_{i}=-\underline{E} \cdot \underline{\mu}=-\hbar \underline{v} \cdot \underline{D},
$$

where

$$
\underline{\mathrm{V}}=(\sqrt{2} p / \hbar) \underline{\mathrm{E}} .
$$

The electric field E will be identified later on with one of the vectorial modes introduced in Chapter 2.

The quantum mechanical state of an atom will, as usual, be described by the density matrix

$$
\rho=\left(\begin{array}{cc}
\rho^{a a} & \rho^{a b} \\
\rho^{b a} & \rho^{b b}
\end{array}\right),
$$


where $\rho^{\text {aa }}$ is a $3 \times 3$ matrix acting in the triplet state, $\rho^{a b}$ is a column vector, relating the upper and lower level; $\rho^{\text {ba }}=\rho^{a b t}$ is a row vector and $\rho^{\mathrm{bb}}$ is a scalar, acting in the singlet state. The time evolution of $\rho$ in the presence of the perturbation $(3-14)$ for an atom at rest is given by the density matrix equation

$$
i \hbar \partial_{t}^{\rho}=\left[H_{0}+H_{i}, \rho\right]+\left.i \hbar \partial t^{\rho}\right|_{\text {stoch }},
$$

where the last term in the right-hand side accounts for effects like collisions, spontaneous emission, radiation trapping, as well as pumping, to be discussed later on. We start by discussing the effect of the commutator term first. By substitution of (3-7), (3-2), (3-14) and (3-16) we obtain, apart from the stochastic term,

$$
\begin{aligned}
& \partial_{t} \rho^{\alpha \alpha}=i \underline{V} \cdot[\underline{D}, \rho]^{\alpha \alpha} \quad(\alpha=a, b) ; \\
& \left(\partial_{t}+i \Omega\right) \rho^{a b}=i \underline{V} \cdot[\underline{D}, \rho]^{a b},
\end{aligned}
$$

where

$$
\Omega=\Omega_{a}-\Omega_{b} \text {. }
$$

The equation for $\rho^{\text {ba }}$ follows from (3-19) by taking hermitian conjugates of both sides of the equality.

We will now derive equations for the expectation values of the observables defined in (3-2) - (3-6). For an operator A, its expectation value will be denoted by the corresponding symbol $\mathrm{A}$, or

$$
A=\operatorname{Tr}(\rho \mathrm{A}),
$$

and consequently,

$$
\partial_{t} A=\operatorname{Tr}\left\{\left(\partial_{t} \rho\right) A\right\}
$$


By insertion of the respective operators (3-2)-(3-6) in (3-22) and by applying (3-18) and (3-19), the following equations can he derived:

$$
\begin{aligned}
& 3 \partial_{t} N_{a}=-\partial_{t} N_{b}=\hat{D} \cdot \underline{V} ; \\
& \partial_{t} \underline{J}=\underline{D} \times \underline{V} ; \\
& \partial_{t} ;=-\underline{D} \otimes \underline{V} ; \\
& \partial_{t} ; \\
& \partial_{t} \hat{D}+\Omega \underline{D}=\underline{J} \times \underline{V} ;
\end{aligned}
$$

As these equations refer to measurable quantities, they give direct insight into the mechanisms by which the perturbation $\underline{V}$ affects the expectation values.

In addition to the influence of $\underline{V}$ on the time evolution of expectation values, we still have to consider the effect of the second term in the right-hand side of (3-17). As far as this term is concerned, relative important contributions originate from elastic and nonelastic collisions as well as radiation trapping (i.e., multiple diffusion of spontaneously emitted radiation). We will not go into the details of these processes now. In this chapter we will follow an approach in which relaxation is introduced in a phenomenological way. In Section 4.2 we will pay attention to the underlying mechanisms.

In gaseous systems, the processes which are responsible for relaxation of the system to equilibrium usually do not result in any orientational preference, i.e. the equilibrium expectation values $\bar{J}, \bar{D}, \overline{\bar{D}}$ and $\overline{\underline{Q}}$ are zero, or the equilibrium state is an isotropic state in which only the scalar observables have nonzero expectation values $\left(\bar{N}_{a}, \bar{N}_{b} \neq 0\right)$. The equations describing the relaxation of the expectation values are then invariant under three-dimensional proper rotations. In fact, our applications will be restricted to such cases. Moreover, the usual assumption will be made that the relaxation rate of each expectation value depends only linearly on the deviation from its equilibrium value. In view of these remarks, 
the equations describing the relaxation can be written as

$$
\begin{aligned}
& \left.\partial t^{N} a\right|_{\text {relax }}=-\lambda_{a}\left(N_{a}-\overline{\mathrm{N}}_{a}\right) ; \\
& \left.\partial t^{\mathbb{N}} \mathrm{b}\right|_{\text {relax }}=-\lambda_{b}\left(N_{b}-\bar{N}_{b}\right) ; \\
& \left.\partial t \underline{J}\right|_{\operatorname{relax}}=-\lambda_{J} \underline{J} ; \\
& \left.\partial_{t} \underline{\underline{Q}}\right|_{\text {relax }}=-\lambda_{Q} \underline{Q} \text {; } \\
& \left.\partial_{t} D\right|_{\text {relax }}=-\lambda_{D} D \text {; } \\
& \left.\partial_{t} \underline{\hat{D}}\right|_{\operatorname{relax}}=-\lambda_{\hat{D}} \underline{\hat{D}} \text {, }
\end{aligned}
$$

where the positive quantities $\lambda_{a}, \lambda_{b}, \lambda_{J}, \lambda_{Q}, \lambda_{D}$ and $\lambda_{\hat{D}}$ are the relaxation constants which are accessible to experimental determination as well as to theoretical calculations. In these equations the effect of an isotropic pumping process can be easily taken into account by taking the equilibrium populations $\bar{N}_{a}$ and $\bar{N}_{b}$ different from their thermal-equilibrium values. In the absence of pumping we will have $\overline{\mathrm{N}}_{\mathrm{a}}<\overline{\mathrm{N}}_{\mathrm{b}}$, but in the case of population inversion we will have $\overline{\mathrm{N}}_{\mathrm{a}}>\overline{\mathrm{N}}_{\mathrm{b}}$.

The right-hand sides of (3-28) - (3-33) should be added to the respective right-hand sides of (3-23) - (3-27) in order to obtain the combined effect on the expectation values of the atomic observables. Note however that (3-23) - (3-27) have been derived for atoms having a fixed position in space. If the atoms under consideration move with velocity $\underline{v}$, we have to make the substitution

$$
\partial_{t} \rightarrow \partial_{t}^{v}=\partial_{t}+\underline{v} \cdot \nabla
$$

In fact, the velocities of atoms in the medium will be distributed according to some distribution function, while each velocity group of atoms fulfils its own set of equations. It will be assumed that the velocity distribution is not influenced by the perturbation $\underline{V}$. Moreover, we assume that the relaxation equations (3-28) - (3-33) are independent of the velocities 
of the atoms. The complete equations for the expectation values of the observables associated with the group of atoms having velocity $\underline{v}$ can then be written as

$$
\begin{aligned}
& \left(\partial_{t}^{v}+\lambda_{a}\right) N_{a}=\lambda_{a} \bar{N}_{a}+\frac{1}{3} \hat{D} \cdot \underline{V} ; \\
& \left(\partial_{t}^{v}+\lambda_{b}\right) N_{b}=\lambda_{b} \bar{N}_{b}-\hat{D} \cdot \underline{V} ; \\
& \left(\partial_{t}^{v}+\lambda_{J}\right) \underline{J}=\underline{D} \times \underline{V} ; \\
& \left(\partial_{t}^{v}+\lambda_{Q}\right) \underline{Q}=-\underline{D} \otimes \underline{V} ; \\
& \left(\partial_{t}^{v}+\lambda_{D}\right) \underline{D}-\Omega \hat{D}=\underline{J} \times \underline{V} ; \\
& \left(\partial_{t}^{v}+\lambda_{\hat{D}}\right) \underline{D}+\Omega \underline{D}=-2\left(N_{a}-N_{b}\right) \underline{V}+\underline{Q} \underline{V} .
\end{aligned}
$$

Until now, we have not specified the perturbing field $\underline{\mathrm{V}}$ in detail, and the above-written equations are rather general. In the next section the equations will be applied to the case of a gaseous medium in the resonator described in Section 2.3, i.e., the case in which the perturbing field has a natural-mode character as given in (2-23).

\section{2 solution of the equations of motion}

With the application to a single-mode laser in mind, the perturbing electric field $\underline{E}=(\hbar / \sqrt{2} p) \underline{V}$ in $(3-14)$ will now be taken such that

$$
\underline{V}(\underline{r}, t)=\frac{1}{2} \underline{V}(t) e^{-i \omega t} \sin (\underline{k} \cdot \underline{r})+c \cdot c \cdot,
$$

corresponding to the case in which only one of the longitudinal modes in (2-23) has a vector amplitude different from zero. In the above equation, $\underline{V}(t)=(\sqrt{ } 2 p / \hbar) \underline{E}(n, t) ; \omega=n \pi c / L$ and $\underline{k} \cdot \underline{r}=k n$, where $k=n \pi / L-\phi_{n} / c$. Note that in $(3-41)$ the small imaginary part $\lambda_{n} / c$ in the wave number $k$ has been omitted. The justification for this is based on the observation 
that the spatial deviation of the true $\underline{V}$ from the purely sinusoidal form in (3-41) will not influence the equations of motion derived in Section 3.1. Namely, the moving atoms experience during their life time $\left(\lambda_{a}^{-1}\right.$ or $\left.\lambda_{b}^{-1}\right)$ relative spatial deviations of the order of $\left\{1-\exp \pm\left(\lambda_{n} v / \lambda_{\alpha} c\right)\right\}$ for $\alpha=a, b$. If we take $\lambda_{n} \simeq \lambda_{\alpha} \simeq 10^{7} \mathrm{~s}^{-1}$ and $v / c \simeq 10^{-5}$, this factor is equal to $10^{-5}$.

In order to find solutions of (3-35)-(3-40) an iteration scheme, allowing for an approximate analytic solution, will be set up. Each expectation value is first expressed as a power series in $\underline{V}$ (or, alternatively, in $p)$. So, if A represents one of the quantities $N_{a}, N_{b}, \underline{J}, \underline{Q}, \underline{D}$ or $\underline{\mathrm{D}}$, then $\mathrm{A}$ is expanded as

$$
A=A^{(0)}+A^{(1)}+A^{(2)}+\ldots,
$$

where $A^{(n)}$ is proportional to $p^{n}$. By equating successively the equal-order terms in (3-35) - (3-40) we obtain the following equations:

$$
\begin{aligned}
& \mathrm{N}_{\mathrm{a}}^{(0)}=\overline{\mathrm{N}}_{\mathrm{a}} ; \mathrm{N}_{\mathrm{b}}^{(0)}=\overline{\mathrm{N}}_{\mathrm{b}} ; \underline{J}^{(0)}=0 ; \underline{\underline{Q}}^{(0)}=0 ; \underline{\mathrm{D}}^{(0)}=0 ; \underline{\mathrm{D}}^{(0)}=0 ; \\
& \mathrm{N}_{\mathrm{a}}^{(1)}=0 ; \mathrm{N}_{\mathrm{b}}^{(1)}=0 ; \underline{\mathrm{J}}^{(1)}=0 ; \underline{\underline{Q}}^{(1)}=0 ; \\
& \left(\partial_{t}^{v}+\lambda_{\mathrm{D}}\right) \underline{\mathrm{D}}^{(1)}-\underline{\Omega}^{(1)}=0 ; \\
& \left(\partial_{t}^{v}+\hat{\lambda}_{\hat{D}}\right) \underline{\hat{D}}^{(1)}+\Omega \underline{\mathrm{D}}^{(1)}=-2\left(\overline{\mathrm{N}}_{\mathrm{a}}-\overline{\mathrm{N}}_{\mathrm{b}}\right) \underline{\mathrm{V}} ;
\end{aligned}
$$

and, for $n \geq 1$,

$$
\begin{aligned}
& \left(\partial_{t}^{v}+\lambda_{\mathrm{a}}\right) \mathrm{N}_{\mathrm{a}}(2 n)=\frac{1}{3} \hat{\mathrm{D}}^{(2 n-1)} \cdot \underline{\mathrm{V}} ; \\
& \left(\partial_{t}^{v}+\lambda_{\mathrm{b}}\right) \mathrm{N}_{\mathrm{b}}^{(2 n)}=-\hat{-}^{(2 n-1)} \cdot \underline{\mathrm{V}} ; \\
& \left(\partial_{t}^{v}+\lambda_{\mathrm{J}}\right) \underline{\underline{J}}^{(2 n)}=\underline{\underline{D}}^{(2 n-1)} \times \underline{\mathrm{V}} ; \\
& \left(\partial_{t}^{v}+\lambda_{\mathrm{Q}}\right) \underline{\underline{Q}}^{(2 n)}=-\underline{-}^{(2 n-1)} \otimes \underline{\mathrm{V}} ;
\end{aligned}
$$




$$
\begin{aligned}
& \left(\partial_{t}^{v}+\lambda_{D}\right) \underline{D}^{(2 n+1)}-\Omega \hat{\underline{D}}^{(2 n+1)}=\underline{J}^{(2 n)} \times \underline{V} ; \\
& \left(\partial_{t}^{v}+\lambda \hat{D}^{\prime} \hat{\underline{D}}^{(2 n+1)}+\Omega \underline{D}^{(2 n+1)}=-2\left(N_{a}^{(2 n)}-N_{b}^{(2 n)}\right) \underline{V}+\underline{Q}^{(2 n)} \underline{V} .\right.
\end{aligned}
$$

As it turns out to be sufficient to carry through the iteration procedure until a third-order expression for $D$ is obtained, only a restricted set of lower order quantities has to be considered.

If we neglect for the moment the weak time dependence of $\underline{V}$, then the coupled equations (3-45) and (3-46) can easily be integrated to obtain the first-order expressions for $\underline{D}$ and $\underline{\hat{D}}$. If we also use the approximations $\Omega^{2}-\omega^{2} \simeq 2 \Omega(\Omega-\omega) ; \underline{k} \cdot \underline{v} / \Omega \simeq 0$ and $\lambda_{D} / \Omega \simeq 0$ (in representative cases one has $\left.\lambda_{D} \leq 10^{9} \mathrm{~s}^{-1} ;|\underline{k} \cdot \underline{v}| \simeq 10^{10} \mathrm{~s}^{-1} ; \Omega \simeq 10^{15} \mathrm{~s}^{-1}\right)$, the expression for $\underline{\mathrm{D}}^{(1)}$ can be written as

$$
\underline{D}^{(1)}=\frac{i}{4} \bar{N}_{a b-V} e^{-i \omega t}\left\{\frac{e^{i \underline{k} \cdot \underline{r}}}{\Omega-\omega+\underline{k} \cdot \underline{v}-i \lambda}-\frac{e^{-\underline{i} \underline{k} \cdot \underline{r}}}{\Omega-\omega-\underline{k} \cdot \underline{v}-i \lambda}\right\}+c \cdot c .,
$$

where

$$
\overline{\mathrm{N}}_{a b}=\overline{\mathrm{N}}_{\mathrm{a}}-\overline{\mathrm{N}}_{\mathrm{b}} \text {, }
$$

and

$$
\lambda=\frac{1}{2}\left(\lambda_{D}+\lambda \hat{D}\right) .
$$

The expression for $\underline{\underline{D}}^{(1)}$ follows from (3-53) and (3-45). Using the abovementioned approximation we obtain

$$
\underline{\underline{D}}^{(1)}=\omega^{-1} \partial_{t} \underline{D}^{(1)} \text {. }
$$

It can be verified that as long as $\underline{V}(t)$ shows substantial variations only during time intervals much larger than $\lambda_{D}^{-1}$ and $\lambda_{\bar{D}}^{-1}$, expressions (3-53) and (3-56) are still approximately correct. In fact, our analysis is restricted here and in the following to cases in which this applies. 
By substitution of (3-53) and (3-56) in the right-hand sides of (3-47)(3-50), taking $n=1$, we can now derive second-order contributions to the quantities $\mathrm{N}_{\mathrm{a}}, \mathrm{N}_{\mathrm{b}}$, $\underline{J}$ and $\underline{\underline{Q}}$. It is observed that the right-hand sides consist of slowly time-dependent terms as well as high-frequency terms at frequency $2 \omega$. As the latter terms give rise to nonresonant response, i.e., lead to contributions to the expectation values which are by an order of $\omega / \lambda$ smaller than those originating from the former terms, we will only consider weakly time-dependent terms in the right-hand sides of (3-47) - (3-50). Another approximation concerns the spatial dependence. After taking the various products of $\underline{V}$ and $\underline{D}$ or $\underline{D}$, we are confronted with $r$ independent terms and terms proportional to $\exp ( \pm 2 \underline{i \underline{k}} \cdot \underline{\underline{p}})$. These latter terms give rise to ripples in the spatial dependence of $\mathrm{N}_{a}, \mathrm{~N}_{\mathrm{b}}, \underline{\mathrm{J}}$ and $\underline{Q}$ which are not necessarily negligible. It turns out, however, that the effect of the oscillatory terms can be neglected in the practical applications we have in mind.

We are thus left with space-independent and nearly time-independent right-hand sides in (3-47) - (3-50). Integration is therefore easy and leads to the following expressions

$$
\begin{aligned}
& \mathrm{N}_{\mathrm{a}}^{(2)}=-\frac{1}{2 L} \overline{\mathrm{N}}_{\mathrm{ab}}\left(\lambda / \lambda_{\mathrm{a}}\right)\left(L_{+}+L_{-}\right) \underline{V} * \underline{V} ; \\
& \mathrm{N}_{\mathrm{b}}^{(2)}=-\left(3 \lambda_{\mathrm{a}} / \lambda_{\mathrm{b}}\right) \mathrm{N}_{\mathrm{a}}^{(2)} ; \\
& \underline{J}^{(2)}=\frac{i}{8} \bar{N}_{\mathrm{ab}}\left(\lambda / \lambda_{J}\right)\left(L_{+}+L_{-}\right) \underline{V}^{*} \times \underline{V} ; \\
& \underline{\underline{Q}}^{(2)}=\frac{1}{8} \overline{\mathbb{N}}_{\mathrm{ab}}\left(\lambda / \lambda_{\mathrm{Q}}\right)\left(L_{+}+L_{-}\right) \underline{V}^{*} \otimes \underline{V} ;
\end{aligned}
$$

where

$$
L_{ \pm}=\left\{(\Omega-\omega \pm \underline{k} \cdot \underline{v})^{2}+\lambda^{2}\right\}^{-1} .
$$

The first two equations describe -in lowest nonvanishing order- the we11known phenomenon of "hole-burning" in the populations [7]. As an additional effect, (3-59) - (3-60) show that the perturbing field induces certain anisotropies in the medium, i.e., finite values of $\underline{\mathrm{J}}$ and $\underline{\underline{Q}}$. Contrary to 
hole burning in the populations, the induced angular- and quadrupolemomenta depend on the state of polarization of the perturbing field. For instance, it follows from (3-59) that $\underline{J}^{(2)}=0$ if $\underline{V}$ is 1 inearly polarized. There is however no state of polarization of $\underline{\mathrm{V}}$ for which ${ }_{\underline{\underline{Q}}}^{(2)}$ vanishes. In general, an elliptically polarized field induces both angular- and quadrupole-momentum.

The effect of the second-order terms on $\underline{D}$ can now be calculated by substitution of (3-57) - (3-61) into (3-51) and (3-52), taking $n=1$ in the latter two equations. Again using the approximations mentioned in connection with the derivation of (3-53), we obtain

$$
\begin{aligned}
\underline{D}^{(3)}= & \frac{1}{32 i} \bar{N}_{a b} \lambda\left(L_{+}+L_{-}\right)\left\{\frac{e^{i \underline{\underline{k}} \cdot \underline{r}}}{\Omega-\omega+\underline{k} \cdot \underline{v}-i \lambda}-\frac{e^{-\underline{i \underline{k}} \cdot \underline{\underline{r}}}}{\Omega-\omega-\underline{k} \cdot \underline{v}-i \lambda}\right\} e^{-i \omega t} \\
& \times\left\{\left(\frac{1}{3 \lambda_{\mathrm{a}}}+\frac{1}{\lambda_{\mathrm{b}}}\right)\left(\underline{V}^{*} \cdot \underline{V}\right) \underline{V}+\frac{1}{2 \lambda_{J}}\left(\underline{V}^{*} \times \underline{V}\right) \times \underline{V}+\frac{1}{2 \lambda_{Q}}\left(\underline{V}^{*} \otimes \underline{V}\right) \underline{V}\right\}+c \cdot c .
\end{aligned}
$$

\section{3 the polarization response}

Let $d$ denote the density of the atoms and $\Phi(\underline{v})$ their velocitydistribution function. The induced dipole-moment density (or polarization) up to third order in the driving electric field is then given by [see also $(3-12)]$

$$
\underline{P}(\underline{r}, t)=\sqrt{ } 2 d \underline{p} \int \underline{d v} \Phi(\underline{v})\left\{\underline{D}^{(1)}(\underline{v})+\underline{D}^{(3)}(\underline{v})\right\} .
$$

We restrict ourselves to the case of a symmetric velocity-distribution function, i.e., $\Phi(\underline{v})=\Phi(\underline{v})$. It then follows from the $\underline{v}$-dependence of $\underline{D}^{(1)}$ and $\underline{D}^{(3)}$, given by $(3-53)$ and $(3-62)$, respectively, that $\underline{P}$ can be written as

$$
\underline{P}(\underline{r}, t)=\frac{1}{2} \underline{P} e^{-i \omega t} \sin (k \cdot \underline{r})+c \cdot c \cdot
$$


We express $\underline{P}$ in the electric-field vector $\underline{E}$, related to $\underline{V}$ by $[$ see $(3-15)]$

$$
\underline{E}=(\sqrt{ } 2 p)^{-1} \hbar \underline{V},
$$

and use (3-9) - (3-10) for the evaluation of the vector products in (3-62). In this way we obtain for the polarization amplitude vector

$$
\left(\omega / \varepsilon_{0}\right) \underline{P}=-a \underline{E}+(b-c)\left(\underline{E} \cdot \underline{E}^{*}\right) \underline{E}+c(\underline{E} \cdot \underline{E}) \underline{E}^{*},
$$

where the coefficients $a, b$ and $c$, introduced in (2-54), appear to be equal to

$$
\begin{aligned}
& a=\frac{2 \omega}{\hbar \varepsilon_{0}} \bar{N} p^{2} K_{0}(\omega-\Omega) ; \\
& b=\frac{\omega}{4 \hbar^{3} \varepsilon_{0}} \bar{N} p^{4}\left(\frac{1}{3 \lambda_{a}}+\frac{2}{3 \lambda_{Q}}+\frac{1}{\lambda_{b}}\right) K(\omega-\Omega) ; \\
& c=\frac{\omega}{8 \hbar^{3} \varepsilon_{0}} \bar{N} p^{4}\left(\frac{1}{\lambda_{Q}}-\frac{1}{\lambda_{J}}\right) K(\omega-\Omega) .
\end{aligned}
$$

Here we have introduced the pump strength $\bar{N}$ by

$$
\overline{\mathrm{N}}=d \overline{\mathrm{N}}_{\mathrm{ab}},
$$

while the $K$ functions are given by

$$
\begin{aligned}
& K_{0}(\omega-\Omega)=\int \underline{\operatorname{d}} \underline{\Phi}(\underline{v}) /(\Omega-\omega+\underline{k} \cdot \underline{v}-i \lambda) ; \\
& K(\omega-\Omega)=2 \int \underline{d} \underline{v} \Phi(\underline{v}) /\left\{\left(L_{+}+L_{-}\right)(\Omega-\omega+\underline{k} \cdot \underline{v}-i \lambda)\right\} .
\end{aligned}
$$

The first term in the right-hand side of (3-66) represents the linear polarization of the medium, the coefficient $a$ being proportional to the linear susceptibility. The imaginary part of a represents the linear gain or loss (if $\bar{N}>0$ or $\bar{N}<0$, respectively) of the medium; its real part reflects the linear dispersion. Their dependencies on $\omega-\Omega$ follow from (3-71) and will be discussed below. The second and third term in 
the right-hand side of (3-66) give rise to lowest-order saturation effects of the medium. Higher-order saturation effects will be disregarded. The second term is a contribution to $\underline{P}$ in the same direction as the driving field $\underline{E}$, whereas the contribution of the third term is oriented along $\underline{E}^{*}$ which generally has a direction different from $\underline{E}$. So, this term can lead to anisotropic saturation effects. The dependencies of both terms on $\omega-\Omega$ follows from the function $K$ given in (3-72). $K$ can be expressed in terms of $K_{0}$, and reads

$$
K(\Delta)=i\left\{\frac{K_{0}^{\prime \prime}(\Delta)}{\lambda}-\frac{K_{0}^{\prime}(\Delta)}{\Delta}+\frac{K_{0}(\Delta)}{\Delta+i \lambda}-\mathrm{d}_{\Delta} K_{0}(\Delta)\right\},
$$

where $\Delta=\omega-\Omega ; K_{0}^{\prime}$ is the real part of $K_{0}$ and $K_{0}^{\prime \prime}$ is the imaginary part. If we specialize to a Maxwellian distribution function, i.e.,

$$
\Phi(\underline{v})=(\sqrt{ } \pi \bar{v})^{-3} \exp \left(-\underline{v}^{2} / \bar{v}^{2}\right)
$$

where $\bar{v}$ is the r.m.s. velocity, $K_{0}$ can be approximated by a simpler expression under the condition that $\lambda<<\bar{k}$. In this limiting case, known as the Doppler limit [63], we have

$$
K_{0}(\Delta)=\frac{1}{k \bar{v}}\left\{2 e^{-(\Delta / k \bar{v})^{2} \Delta / k \bar{v}} \int_{0}^{2} d s e^{s^{2}}+i \sqrt{ } \pi e^{-(\Delta / k \bar{v})^{2}}\right\} .
$$

In Figure 3.1 plots are given of the real and imaginary parts of $K_{0}$ and $K$, calculated by means of the approximate expression (3-75) for $K_{0}$. The imaginary part of $\mathrm{K}^{\prime \prime}$ reaches a sharp local maximum for $\omega-\Omega=0$, indicating that the saturation effects become rough1y twice as effective when the field frequency is tuned to the atomic transition frequency. This we11known phenomenon is due exclusively to the standing-wave character of the driving field.

The coefficients $b$ and $c$ are seen to be additive in terms that are inversely proportional to the relaxation constants [see (3-68) and (3-69)]. This shows their saturating character, since the lower the relaxation process, the slower the replenishment of the atoms involved by freshly 


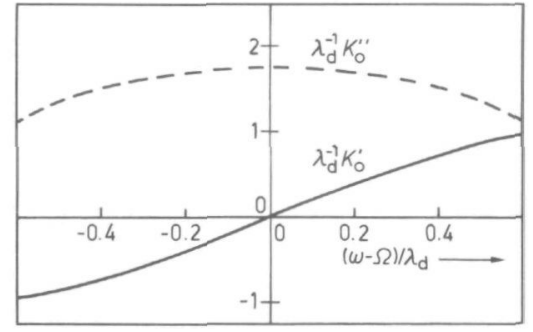

(a)

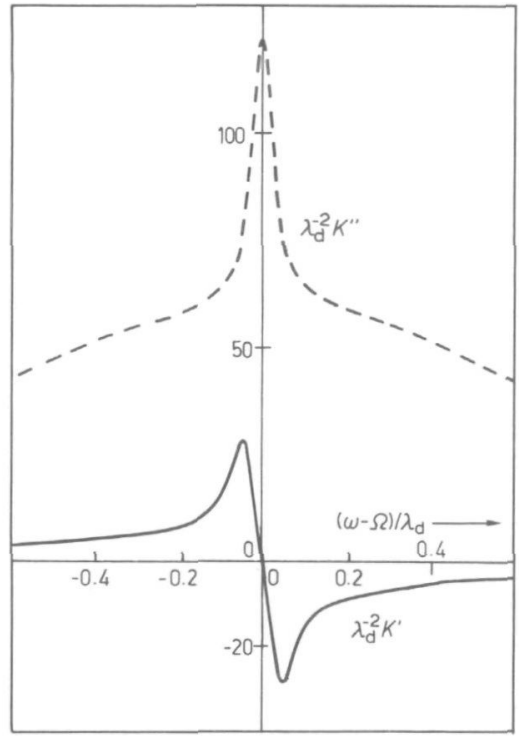

(b)

Fig.3.1 Real (-) and imaginary (---) parts of (a) $K_{0}$ and (b) $K$ versus detuning frequency for a case in which $\lambda / \lambda_{\mathrm{d}}=0.0287$, where $\lambda_{\mathrm{d}} \equiv k \bar{v}$

excited ones will take place and, therefore, the larger the saturation effects will be. We conclude from (3-69) that the $1 \rightarrow 0$ transition can show anisotropic polarization response only if angular momentum and quadrupole momentum have different relaxation rates. This was already shown by Polder \& Van Haeringen [27].

The analysis given here for the $j=1 \rightarrow j=0$ transition is directly applicable to the $0 \rightarrow 1$ case as well. In fact, if the following substitutions are made:

$$
\begin{aligned}
& \Omega \rightarrow-\Omega ; \\
& \omega \rightarrow-\omega ; \\
& \bar{N} \rightarrow-\bar{N} ; \\
& \underline{E} \rightarrow \underline{E}^{*} ; \\
& \underline{P} \rightarrow \underline{P}^{*} ;
\end{aligned}
$$


the analysis can be performed in exactly the same way. Using the properties

$$
\begin{aligned}
& K_{0}^{*}(\omega-\Omega)=-K_{0}(\Omega-\omega) ; \\
& K^{*}(\omega-\Omega)=-K(\Omega-\omega),
\end{aligned}
$$

it can be verified that the same $a, b$ and $c$ coefficients are obtained as in (3-67) - (3-69), in which as a matter of course the subscripts $\mathrm{a}$ and $\mathrm{b}$ have to be interchanged. 
4 THE $1 \rightarrow 0$ TRANSITION LASER

The theory developed in the preceding chapter enables us to discuss the polarization behaviour of a gas laser in which a $j=1 \rightarrow j=0$ atomic transition is involved. Historically, this kind of transition is of special interest, as the corresponding experimentally observed "saturationinduced polarization preference" for circular polarization [26] could not be explained in terms of the developed nonscalar theory of laser operation, though on the other hand the polarization preferences observed for other transitions appeared to be in good agreement with the theory $[18,25,39]$.

Polder \& Van Haeringen [27] pointed out in 1967 that the observed preference could be an effect due to different relaxation rates of angular momentum and quadrupole momentum. However, they gave no explicit calculations, while furthermore the precise connection with collisional processes obtained only marginal attention. The present chapter will be entirely devoted to the discussion of the saturation-induced polarization phenomena in a $j=1 \rightarrow j=0$ transition laser.

In Section 4.1 the equations for the time evolution of polarization parameters are given. Stable, time-independent solutions are found and related to the experimentally observed polarization. In Section 4.2 a collision model is analyzed in some detail, which leads to expressions for the relaxation constants of angular and quadrupole momentum. It will be shown that the polarization behaviour observed in $1 \rightarrow 0$ lasers is in qualitative agreement with the theoretical predictions.

\section{1 behaviour of the polarization parameters}

The axial symmetry in a laser, free from externally applied fields, favours a representation of the state of polarization of the laser field in terms of the ellipticity $x$ and the azimuth $U$ (see Section 2.4). The equations for the time evolutions of $x, u$ and $I$ for the (artificial) case of an isotropic laser (no mirror anisotropies) can be obtained from 
$(2-48)-(2-50)$, firstly by substituting the appropriate resonator-induced contributions, i.e., taking $\Gamma_{a}=\Gamma_{p}=\Gamma_{c}=0$ in $(B-6)-(B-8)$ of Appendix $B$, and secondly by substituting the medium-induced contributions (2-51) (2-53), i.e., taking $a_{1}=b_{1}=b_{2}=0$ in the present magnetic-field-free case. The equations obtained read

$$
\begin{aligned}
& \mathrm{a}_{t} \mathrm{x}=\frac{1}{4} c^{\prime \prime} I \sin 4 x ; \\
& \cos 2 x \mathrm{~d}_{t^{u}}{ }^{\mathrm{u}}=\frac{1}{4} c^{\prime} I \sin 4 x ; \\
& I^{-1} \mathrm{~d}_{t^{\prime}} I=a^{\prime \prime}-\Gamma^{\prime}-I\left(b^{\prime \prime}-c^{\prime \prime} \sin ^{2} 2 x\right) .
\end{aligned}
$$

The $a, b$ and $c$ coefficients in these equations have been calculated in the preceding chapter for the $1 \rightarrow 0$ type of transition. Expressions for them are given in (3-67)-(3-69).

Laser operation follows if the linear gain $a^{\prime \prime}-\Gamma^{\prime}>0$. In that case the equations (4-1) - (4-3) lead to time-independent solutions for the polarization parameters, of which the value of the azimuth $U$ is undetermined, or more precisely, determined by the unknown starting conditions in the history of the laser. The value taken by the ellipticity $x$ depends on the sign of the coefficient $c$ ", i.e.,

$$
\begin{aligned}
& x=0 \quad \text { if } c^{\prime \prime}<0 \text {, } \\
& x= \pm \pi / 4 \quad \text { if } c^{\prime \prime}>0 \text {, } \\
& x \text { undetermined if } c^{\prime \prime}=0 \text {, } \\
& \left.\begin{array}{r}
\text { (1inear polarization); } \\
\text { (circular polarization); } \\
\text { (arbitrary polarization) }
\end{array}\right\}
\end{aligned}
$$

The intensity $I$ assumes the stationary value

$$
I=\left(a^{\prime \prime}-\Gamma^{\prime}\right) /\left(b^{\prime \prime}-c^{\prime \prime} \sin ^{2} 2 x\right)
$$

For the $j=1 \rightarrow j=0$, Ne $1.523 \mu$ transition, De Lang \& Bouwhuis [26] experimentally observed circular polarization of the mode, caused by the response of the medium. The theory developed thusfar should therefore at least be extended in order to determine the sign of the constant $c$ ". 
A condition necessary to explain the observed polarization phenomenon is clearly $c^{\prime \prime}>0$, or [ see (3-69)]

$$
\lambda_{J}>\lambda_{Q}
$$

This implies that the angular momentum should show a faster relaxation than the quadrupole momentum.

De Lang \& Bouwhuis did not only determine the kind of the polarization preference, but tried to measure its "strength" as well. The measuring method can best be illustrated by considering (4-1) for the case in which $x$ and $I$ are close to their time-independent values, respectively. In that case it is allowed to substitute (4-5) into (4-1), which leads to the equation

$$
a_{t} x=\frac{1}{2}\left(n_{e}-1\right) \Gamma^{\prime} \frac{\sin 4 x}{T+\cos 4 x}
$$

where the excitation density $\eta_{e}$ is defined by

$$
\eta_{e}=a^{\prime \prime} / \Gamma^{\prime},
$$

and where

$$
T=\left(2 b^{\prime \prime}-c^{\prime \prime}\right) / c^{\prime \prime}
$$

Equation (4-7) has first been given by De Lang et al. [24], and has a greater validity than for the case of $1 \rightarrow 0$ transitions only, since other types of transitions only lead to different expressions for $a, b$ and $c$. By balancing the driving term in the right-hand side of (4-7) with a given external anisotropy term, De Lang \& Bouwhuis were able to give a rough estimate for the value of the quantity T. For the Ne $1.523 \mu \mathrm{m}$ transition case, they found $[26,36]$ 
Using (3-68) and (3-69), we can express the right-hand side of (4-9) in terms of three ratios $\lambda_{Q} / \lambda_{a}, \lambda_{Q} / \lambda_{b}$ and $\lambda_{Q} / \lambda_{J}$. The result is

$$
T=\frac{\frac{4 \lambda_{Q}}{3 \lambda_{a}}+\frac{4 \lambda_{Q}}{\lambda_{b}}+\frac{\lambda_{Q}}{\lambda_{J}}}{1-\frac{\lambda_{Q}}{\lambda_{J}}} .
$$

Experimental verification of (4-11) implies therefore not only the determination of $\lambda_{Q} / \lambda_{J}$, but also that of $\lambda_{Q} / \lambda_{a}$ and $\lambda_{Q} / \lambda_{b}$. Measurements of $\lambda_{J}$ in a He-Ne gas mixture have not been reported to our knowledge, but fairly accurate measurements of $\lambda_{a}$ and $\lambda_{Q}$, as well as their dependences on the partial He and Ne pressures, have been performed by Decomps \& Dumont [55]. For partial He and Ne pressures of 4.6 Torr and 0.4 Torr, respectively, we obtain from these measurements $\lambda_{Q} / \lambda_{a}=3.8( \pm 0.1)$ and $\lambda_{Q}=9.4( \pm 1) 10^{7} \mathrm{~s}^{-1}$. For $\lambda_{b}$ we assume the value $6.8( \pm 0.7) 10^{7} \mathrm{~s}^{-1}$, which corresponds to the life-time measurement of the Ne $2 \mathrm{p}_{1}$ leve1 by Klose [56]. This leads to $\lambda_{Q} / \lambda_{b}=1.4( \pm 0.3)$. By taking successively the right-hand side of $(4-11)$ equal to the values given in (4-10), we conclude that

$$
1.20 \leq \lambda_{J} / \lambda_{Q} \leq 2.30
$$

In the next section we will derive a theoretical estimate of the quantity $\lambda_{J} / \lambda_{Q}$ by taking into account collision processes that cause the largest contributions to $\lambda_{J}$ and $\lambda_{Q}$. It will be shown that the theoretically obtained ratio of $\lambda_{J}$ and $\lambda_{Q}$ indeed fits within the inequality (4-12). However, the described confrontation of the theory with experiment suffers heavily from the poorly determined $T$ value $[$ see (4-10)]. A more accurate value of $T$ is needed to enclose $\lambda_{J} / \lambda_{Q}$ between narrower bounds. 
4.2 calculation of $\lambda_{J}$ and $\lambda_{Q}$

In this section we intend to give an account of the respective relaxation phenomena relevant for the determination of the ratio $\lambda_{J} / \lambda_{Q}$. Fortunately, a large amount of work has been done already on this subject during the last 15 years, both theoretically and experimentally. Detailed lists of references can be found in the review articles by Happer [57] and by Omont [58]. Without pretending completeness, we will reconsider those parts of the existing atomic collision theory which are relevant in explaining a difference in respective multipole relaxation rates.

Let $\rho$ be the partial density matrix corresponding to a multiplet state with angular-momentum quantum number $j$. Though our prime interest is in atomic multiplet states with $j=1$, the analysis can in principle be carried through for an arbitrary value of $j$. The occurrence of different relaxation rates implies that the picture in which the matrix elements of $\rho$ all tend to reach their equilibrium values by equal rates is incorrect. The $\rho$-matrix can be shown to be decomposable into $2 j+1$ moments, each of which corresponds to a particular multipole moment $[57,58]$, or,

$$
\rho=\rho^{(0)}+\rho^{(1)}+\rho^{(2)}+\ldots+\rho^{(2 j)},
$$

where $\rho^{(0)}$ corresponds to the global population of the multiplet; $\rho^{(1)}$ to the angular momentum; $\rho^{(2)}$ to the quadrupole momentum, etc. General expressions for these moments can be found in Reference [58].

If the relaxation is an isotropic process, that is, if the relaxation equations are invariant under three-dimensional proper rotations, which we will assume, then to each multipole moment $\rho^{(M)}$ of $\rho$ there can be assigned a relaxation constant $\lambda^{(M)}$. The value of $\lambda^{(M)}$ should follow from a study of all randomizing mechanisms by which $\rho$ can change in time. In view of our purpose we will confine ourselves to the determination of $\lambda^{(1)}$ and $\lambda^{(2)}$ for the $j=1$ triplet. In that case, one has explicitly [58], in the representation adopted in Chapter 3 , 


$$
\begin{aligned}
& \rho^{(0)}=\frac{1}{3}(\operatorname{Tr} \rho)\left(\begin{array}{lll}
1 & 0 & 0 \\
0 & 1 & 0 \\
0 & 0 & 1
\end{array}\right) ; \\
& \rho^{(1)}=\frac{1}{2}\left(\begin{array}{ccc}
\rho_{11}-\rho_{-1-1} & \rho_{10}+\rho_{0-1} & 0 \\
\rho_{01}+\rho_{-10} & 0 & \rho_{10}+\rho_{0-1} \\
0 & \rho_{01}+\rho_{-10} & -\rho_{11}+\rho_{-1-1}
\end{array}\right) ; \\
& \rho^{(2)}=\frac{1}{2}\left(\begin{array}{ccc}
\frac{1}{3} \operatorname{Tr} \rho-\rho_{00} & \rho_{10}-\rho_{0} 1 & 2 \rho_{1-1} \\
\rho_{01}-\rho_{-10} & -2\left(\frac{1}{3} \operatorname{Tr} \rho-\rho_{00}\right) & -\rho_{10}+\rho_{0-1} \\
2 \rho_{-11} & -\rho_{01}+\rho_{-10} & \frac{1}{3} \operatorname{Tr} \rho_{-}-\rho_{00}
\end{array}\right) .
\end{aligned}
$$

The mechanisms which give the largest contributions to $\lambda^{(1)}$ and $\lambda^{(2)}$ are $[30,55,57]:$ (1) Van der Waals collisions between identical atoms (in the He-Ne gas mixture, collisions between Ne atoms); (2) Van der Waals collisions between different atoms (collisions between $\mathrm{Ne}$ and He atoms) and (3) radiation trapping. The first-mentioned mechanism concerns induced dipole-dipole interactions between two identical atoms, one of which is in the $j=1$ state, the other of which is in another state such that electricdipole transitions between these two states are allowed. The second mechanism concerns similar interactions between nonidentical atoms. Radiation trapping is the multiple diffusion of radiation by reabsorbtion of a spontaneously emitted photon by another atom of the gas. In the $\mathrm{He}-\mathrm{Ne}$ gas laser at moderate Ne pressure ( 1 Torr) radiation trapping results in a considerable lengthening of the life time of the $2 s_{2}$ Ne 1evel[30,57]. Let us consider the first-mentioned collision process in more detail. The two colliding, identical atoms will be denoted by A and B. Suppose one of them, say A, is initially in one of the three $j=1$ substates and let the other be in a (the) singlet state. We denote by $\mid A, 1, m ; B, 0,0>$ the wave function corresponding to the state in which A occupies the $m$-th magnetic sublevel of the $j=1$ state and $B$ the $j=0$ level. The wave function $\mid A, 0,0 ; B, 1, m>$ is assigned to the state in which $A$ and $B$ have changed their roles. During the collision process the wave function of the two-atom system will be some linear combination: 


$$
\Psi_{A B}=\sum_{m=0, \pm 1}\left\{A_{m}(t)\left|A, 1, m ; B, 0,0>+B_{m}(t)\right| A, 0,0 ; B, 1, m>\right\} e^{-i\left(\Omega_{1}+\Omega_{0}\right) t}
$$

where $\hbar_{\Omega_{1}}$ and $\hbar_{\Omega_{0}}$ are the energies of the $j=1$ and $j=0$ states, respectively. The interaction potential reads $[28,29]$

$$
V(\underline{R})=\left(4 \pi \varepsilon_{0} R^{3}\right)^{-1}\left\{3\left(\underline{\mu}_{A} \cdot \underline{\hat{u}}\right)\left(\underline{\mu}_{B} \cdot \underline{\hat{u}}\right)-\underline{\mu}_{A} \cdot \underline{\mu}_{B}\right\},
$$

where $R=|R| ; \hat{u}=R / R$ and $R$ is the distance vector joining the two atoms. $\underline{\mu}_{A}$ and $\underline{\mu}_{B}$ are the electric-dipole operators of atoms $A$ and $B$, respectively. The matrix representations of $\underline{\mu}_{A}$ and $\underline{\mu}_{B}$ can be deduced from (3-12) and (3-4). Application of Schrödinger's equation leads to equations for the time evolution of the $A_{m}$ and $B_{m}$. If we denote the only nonvanishing matrix elements of $V(R)$ by

$$
\begin{aligned}
V_{m m^{\prime}} & =\left\langle\mathrm{A}, 1, m ; \mathrm{B}, 0,0|V(\underline{R})| \mathrm{A}, 0,0 ; \mathrm{B}, 1, m^{\prime}\right\rangle \\
& =\left\langle\mathrm{A}, 0,0 ; \mathrm{B}, 1, m|V(\underline{R})| \mathrm{A}, 1, m^{\prime} ; \mathrm{B}, 0,0\right\rangle,
\end{aligned}
$$

the resulting equations read

$$
\begin{aligned}
& i \hbar \mathrm{d}_{t^{A}{ }_{m}}=\sum_{m}, v_{m m^{\prime}}{ }^{B_{m}}, ; \\
& i \hbar \mathrm{d}_{t} B_{m}=\sum_{m}, v_{m m^{\prime}} A_{m^{\prime}}
\end{aligned}
$$

The matrix elements $V_{m m^{\prime}}$ can easily be calculated by using the matrix representations (3-4), and the result can be written as

$$
V_{m m^{\prime}}=\frac{9 \hbar \lambda}{4 k_{10}^{3} R^{3}}\left\{(\underline{I} \cdot \underline{\hat{u}})^{2}-\frac{2}{3} 1\right\}_{m m^{\prime}}
$$

where $\lambda_{\text {sp }}$ is the inverse life time for a spontaneous transition [59],

$$
\lambda_{\mathrm{sp}}=\frac{1}{4 \pi \varepsilon_{0}} \frac{4 k_{10}^{3} p^{2}}{9 \hbar} .
$$


In (4-21) $k_{10}$ is the wave number of the transition; $I$ is the angular momentum with components

$$
J_{x}=\frac{1}{\sqrt{2}}\left(\begin{array}{lll}
0 & 1 & 0 \\
1 & 0 & 1 \\
0 & 1 & 0
\end{array}\right) ; J_{y}=\frac{1}{\sqrt{2}}\left(\begin{array}{ccc}
0 & -i & 0 \\
i & 0 & -i \\
0 & i & 0
\end{array}\right) ; J_{z}=\left(\begin{array}{ccc}
1 & 0 & 0 \\
0 & 0 & 0 \\
0 & 0 & -1
\end{array}\right) ;
$$

and 1 is the $3 \times 3$ unit matrix.

As far as the distance vector $\underline{R}(t)$ of the atoms is concerned, we will describe the collision process classically, i.e., we will take

$$
\underline{R}(t)=\underline{b}+\underline{v} t,
$$

where $\underline{b}$ is the impact parameter of the collision and $\underline{v}$ is the relative velocity of the two atoms $(\underline{b} \underline{v})$. It is convenient to introduce the dimensionless parameters

$$
\begin{array}{ll}
\tau=v t / R(t), & (-1 \leq \tau \leq 1) ; \\
\sigma=\frac{9 \lambda \mathrm{sp}}{4 k_{10}^{3} b^{2} v}, & (\sigma>0) .
\end{array}
$$

If we then take $\underline{b}$ along $\underline{\hat{x}}$ and $\underline{v}$ along $\underline{\hat{z}}$, where $\underline{\hat{x}}$ and $\underline{\hat{z}}$ are unit vectors along the $x^{-}$and $z$-axis, respectively, (4-20) can be transformed into

$$
\begin{aligned}
& {\operatorname{id} A_{\tau} A_{m}}=\sigma \sum_{m^{\prime}} \omega_{m m^{\prime}}(\tau) B_{m^{\prime}} ; \\
& \operatorname{id}_{\tau} B_{m}=\sigma \sum_{m^{\prime}} \omega_{m m^{\prime}}(\tau) A_{m^{\prime}},
\end{aligned}
$$

where $W$ is given by

$$
W(\tau)=\left(\begin{array}{ccc}
\left(3 \tau^{2}-1\right) / 6 & \tau \sqrt{ }\left(1-\tau^{2}\right) / \sqrt{ } 2 & \left(1-\tau^{2}\right) / 2 \\
\tau \sqrt{ }\left(1-\tau^{2}\right) / \sqrt{ } 2 & \left(1-3 \tau^{2}\right) / 3 & -\tau \sqrt{ }\left(1-\tau^{2}\right) / \sqrt{ } 2 \\
\left(1-\tau^{2}\right) / 2 & -\tau \sqrt{ }\left(1-\tau^{2}\right) / \sqrt{ } 2 & \left(3 \tau^{2}-1\right) / 6
\end{array}\right)
$$


It can be verified that the coupled equations (4-27) can be solved analytically only for $W$ matrices that commute at different $\tau$. In that case, the solution of (4-27), satisfying $A_{m}(\tau=-1)=A_{m}(-1)$ and $B_{m}(\tau=-1)=0$, would be given by

$$
\begin{aligned}
& A_{m}(\tau)=\sum_{m^{\prime}} A_{m m^{\prime}}(\tau) A_{m},(-1) ; \\
& B_{m}(\tau)=\sum_{m^{\prime}} B_{m m^{\prime}}(\tau) A_{m}(-1),
\end{aligned}
$$

where

$$
\begin{aligned}
& A(\tau)=\cos \left\{\sigma \int_{-1}^{\tau} d \tau^{\prime} \omega\left(\tau^{\prime}\right)\right\} ; \\
& B(\tau)=-i \sin \left\{\sigma \int_{-1}^{\tau} d \tau^{\prime} \omega\left(\tau^{\prime}\right)\right\} .
\end{aligned}
$$

However, the $W$ matrices given by $(4-28)$ do generally not commute at different $\tau$, so that an exact solution of (4-27) can only be obtained by using a numerical method. Before doing so, let us first treat the method followed by Omont [28], who disregards the noncommutivity of $W$ and proceeds with the approximate solution given by (4-29) - (4-32).

Let $\rho(-1)$ be the density matrix of the $j=1$ triplet before the collision; then the net change of $\rho$, due to one collision with fixed $\underline{b}$ and $\underline{v}$, can be written as

$$
\Delta \rho=\rho(1)-\rho(-1),
$$

where

$$
\rho(1)=A(1) \rho(-1) A(1)^{\dagger}+B(1) \rho(-1) B(1)^{\dagger}
$$

The first term in the right-hand side of (4-34) accounts for the loss in the observable quantities due to a transition of atom A to the $j=0$ state, while the second term reflects the regain of observable quantities associated with the simultaneous, reversed transition of atom $B$. It is evident that for any scalar observable quantity (population or energy) 
this will not lead to a net change at all. For nonscalar observables, i.e., the angular and quadrupole momentum, it will however be shown that the collision in general will lead to a net change of the corresponding expectation values.

The total increment of $\rho$ per unit time is given by

$$
\mathrm{d}_{t} \rho=d \int \mathrm{d}_{\underline{\underline{v}} \underline{\underline{b}} \underline{\underline{v}}} \int \mathrm{d} \underline{b} v \Phi(\underline{v}) \Delta \rho,
$$

where $d$ is the density of atoms $B$, and $\Phi(\underline{v})$ is the normalized distribution function of relative velocities, which we will assume to be independent of the orientation of $\underline{v}$. If $\psi_{b}$ is the polar angle of $\underline{b}$ in a plane perpendicular to $\underline{v}$ and $\Omega_{v}$ is the solid angle of $\underline{v}$, then we can write (4-35) as

$$
a_{t} \rho=a \int_{0}^{\infty} d v \int_{0}^{\infty} d b \quad \int_{0}^{4 \pi} d \Omega_{v} \int_{0}^{2 \pi} d \psi_{b} b v^{3} \Phi(\underline{v}) \Delta \rho
$$

The integration over $\Omega_{v}$ in (4-36) can most easily be performed by using group-theoretical techniques $[28,58]$. For the present $j=1$ case, the result can be summarized as follows: For $M=0,1$ or 2 , let $\rho^{(M)}(-1)$ and $\Delta \rho^{(M)}$ be the multipole moments of $\rho(-1)$ and $\Delta \rho$, respectively; then we derive after a lengthy derivation (found in References $[28,58]$ ) the result

$$
\frac{1}{4 \pi} \int \mathrm{d} \Omega \Omega_{v} \Delta \rho^{(M)}=\left\{P_{\mathrm{A}}^{(M)}+P_{\mathrm{B}}^{(M)}-1\right\} \rho^{(M)}(-1),
$$

where the $P_{\mathrm{A}}^{(M)}$ are given by

$$
\begin{aligned}
& P_{\mathrm{A}}^{(0)}=\frac{1}{3}\left\{N_{\mathrm{A}}^{(0)}+N_{\mathrm{A}}^{(1)}+N_{\mathrm{A}}^{(2)}\right\} ; \\
& P_{\mathrm{A}}^{(1)}=\frac{1}{3} N_{\mathrm{A}}^{(0)}+\frac{1}{6} N_{\mathrm{A}}^{(1)}-\frac{1}{6} N_{\mathrm{A}}^{(2)} ; \\
& P_{\mathrm{A}}^{(2)}=\frac{1}{3} N_{\mathrm{A}}^{(0)}-\frac{1}{6} N_{\mathrm{A}}^{(1)}+\frac{1}{30} N_{\mathrm{A}}^{(2)},
\end{aligned}
$$

while the $P_{\mathrm{B}}^{(M)}$ are defined similarly, i.e., by replacing $\mathrm{A}$ by $\mathrm{B}$ in $(4-38)$, and where 


$$
\begin{aligned}
& N_{\mathrm{A}}^{(0)}= \frac{1}{3}\left|A_{11}(1)+A_{00}(1)+A_{-1-1}(1)\right|^{2} ; \\
& N_{A}^{(1)}= \frac{1}{2}\left\{\left|A_{01}(1)+A_{-10}(1)\right|^{2}+\left|A_{11}(1)-A_{-1-1}(1)\right|^{2}\right. \\
&\left.+\left|A_{10}(1)+A_{0-1}(1)\right|^{2}\right\} ; \\
& N_{A}^{(2)}=\left|A_{1-1}(1)\right|^{2}+\left|A_{-11}(1)\right|^{2}+\frac{1}{2}\left|A_{01}(1)-A_{-10}(1)\right|^{2} \\
&+\frac{1}{2}\left|A_{10}(1)-A_{0-1}(1)\right|^{2}+\frac{1}{6}\left|A_{11}(1)+A_{-1-1}(1)-2 A_{00}(1)\right|^{2},
\end{aligned}
$$

with similar expressions for $N_{\mathrm{B}}^{(M)}$, to be obtained by replacing $A(1)$ by $B(1)$ in (4-39). Explicit expressions for $A(1)$ and $B(1)$ are to be determined from $(4-31),(4-32)$ and $(4-28)$. We find

$$
\begin{aligned}
& A(1)=\left(\begin{array}{rrr}
\cos \frac{2}{3} \sigma & 0 & 0 \\
0 & 1 & 0 \\
0 & 0 & \cos \frac{2}{3} \sigma
\end{array}\right) ; \\
& B(1)=-i\left(\begin{array}{rrr}
0 & 0 & \sin \frac{2}{3} \sigma \\
0 & 0 & 0 \\
\sin \frac{2}{3} \sigma & 0 & 0
\end{array}\right) .
\end{aligned}
$$

We calculate $P_{\mathrm{A}}^{(M)}$ and $P_{\mathrm{B}}^{(M)}$ by substitution of the matrix elements given in (4-40) and (4-41) into (4-39) and by substitution of the resulting $N_{\mathrm{A}}$ 's and $N_{\mathrm{B}}$ 's into (4-38). If we define quantities $P^{(M)}$ by

$$
P^{(M)}=1-P_{\mathrm{A}}^{(M)}-P_{\mathrm{B}}^{(M)},
$$

then, according to $(4-37)$, each $P^{(M)}$ is a measure for the rate of change of the corresponding multipole moment $\rho^{(M)}(-1)$. Their values are given by 


$$
\begin{aligned}
& P^{(0)}=0 ; \\
& P^{(1)}=\frac{4}{3}\left\{3 \sin ^{2}(\sigma / 3)-2 \sin ^{4}(\sigma / 3)\right\} ; \\
& P^{(2)}=\frac{3}{5} P^{(1)} .
\end{aligned}
$$

The first equality in (4-43) is a direct consequence of the conservation of energy during the collision proces. Substituting (4-37) into (4-36), and using $(4-42)$, we can write the rate of change of $\rho^{(M)}$ as

$$
\mathrm{d}_{t^{\rho}}{ }^{(M)}=-4 \pi \int_{0}^{\infty} \mathrm{d} v \int_{0}^{\infty} d b \int_{0}^{2 \pi} d \psi_{b} d b v^{3} \Phi(\underline{v}) P^{(M)} \rho^{(M)}(-1) .
$$

The $\psi_{b}$ integration gives a factor $2 \pi$. Next, using (4-26) we can transform $(4-44)$ into

$$
\mathrm{d}_{t} \rho^{(M)}=-\frac{9 \pi \lambda \mathrm{sp}^{d}}{4 k_{10}^{3}} \int_{0}^{\infty} \mathrm{d} v 4 \pi v^{2} \Phi(\underline{v}) \int_{0}^{\infty} \mathrm{d} \sigma \frac{P^{(M)}}{\sigma^{2}} \rho^{(M)}(-1),
$$

where the remaining integration over $v$ is seen to result in a factor one. Thus we arrive at the following expression for the relaxation constants $\lambda^{(1)}$ and $\lambda^{(2)}$ :

$$
\lambda^{(M)}=\frac{9 \pi \lambda \operatorname{sp}^{d}}{4 k_{10}^{3}} \int_{0}^{\infty} d \sigma P^{(M)} / \sigma^{2}, \quad(M=1,2) .
$$

Substitution of (4-43) and elementary integration over o leads to the final result

$$
\begin{aligned}
& \lambda^{(1)}=0.040 \lambda_{\mathrm{sp}}\left(2 \pi / k_{10}\right)^{3} ; \\
& \lambda^{(2)}=0.024 \lambda_{\mathrm{sp}}\left(2 \pi / k_{10}\right)^{3},
\end{aligned}
$$

or $\lambda^{(1)} / \lambda^{(2)}=5 / 3$. Note that the quantity $2 \pi / k_{10}$ is equal to the wave length corresponding to the $j=1 \rightarrow j=0$ transition. We recall that the values in (4-47) are obtained under the incorrect supposition that two matrices at different $\tau$ are commuting. 
Exact values can be obtained by numerical integration of (4-27) for different choices of $\sigma$. The same procedure as outlined above can then be followed, which leads to values for $P^{(1)}$ and $P^{(2)}$. The constants $\lambda^{(1)}$ and $\lambda^{(2)}$ follow from the calculation of the integral in (4-46). Along this line, we have obtained the values

$$
\begin{aligned}
& \lambda^{(1)}=0.035 \lambda_{\mathrm{sp}}\left(2 \pi / k_{10}\right)^{3} d ; \\
& \lambda^{(2)}=0.029 \lambda_{\mathrm{sp}}\left(2 \pi / k_{10}\right)^{3} d,
\end{aligned}
$$

or $\lambda^{(1)} / \lambda^{(2)}=1.21$, in good agreement with a result obtained by D'Yakonov \& Perel [29]. Note that the approximation carried through by Omont overestimates the ratio $\lambda^{(1)} / \lambda^{(2)}$.

Other relaxation mechanisms can also be treated by following precisely the same procedure, i.e., by first determining the density matrix after the collision, by carrying out the orientational averaging and finally by calculating the increment of $\rho$. An approximate result for the case of induced dipole-dipole interaction between different atoms $A$ and $B$ has been obtained by Omont [28]. In his model, atom A is initially in the $j=1$ state, while during the collision A makes transitions to intermediate states. This is accounted for by an effective potential acting entirely within the three-dimensional $j=1$ triplet. Thus, after the collision, atom A is in the $j=1$ state again, but generally a redistribution over the magnetic sublevels has taken place. Omont's approximation leads again to $\lambda^{(1)} / \lambda^{(2)}=5 / 3$, but should be regarded as an overestimation for the same reason mentioned above.

Let us next mention the effect of radiation trapping on the relaxation constants. This has been calculated by, among others, D'Yakonov \& Pere1 [60]. For the case of complete imprisonment of the spontaneously emitted photon (which is actually the case in a He-Ne laser at moderate pressures), the contribution of radiation trapping to the ralaxation constants $(1 \rightarrow 0$ case) is given by

$$
\begin{aligned}
& \lambda^{(1)}=\frac{1}{2} \lambda_{\text {sp }} ; \\
& \lambda^{(2)}=\frac{3}{10} \lambda_{\text {sp }},
\end{aligned}
$$


i.e., their ratio $\lambda^{(1)} / \lambda^{(2)}=5 / 3$.

It can be proven, by using (4-38) and (4-39), that quite generally the following inequality holds ( $j=1$ case) independently of the nature of the relaxation mechanism:

$$
1 / 3 \leq \lambda^{(1)} / \lambda^{(2)} \leq 5 / 3 .
$$

The physical significance of the lower bound $1 / 3$ has been discussed by D'Yakonov \& Pere1 [61]. They showed that $\lambda^{(1) / \lambda^{(2)}}<1 / 3$ would correspond to the occurrence of negative populations. It is noteworthy that the upper bound $5 / 3$ shows up in some of the above calculations, though, in view of the "exact" result the actual ratio will be definitely smaller. Incidentally, inequalities similar to (4-50) have been derived for levels with arbitrary values of $j$ as we11 [58].

In conclusion, we can state that the theoretical analysis of the various mechanisms contributing to the relaxation of angular and quadrupole momentum definitely confirms that $\lambda^{(1)}>\lambda^{(2)}$. Quantitatively, the abovederived values for $\lambda_{J} / \lambda_{Q}$, being 1.21 for purely elastic collisions or 1.67 for radiation trapping, both fit in the inequality (4-12). The true $\lambda_{J} / \lambda_{Q}$ ratio will be some weighted average between these values. In this connection, it would be of interest if a more accurate experimental method could be developed to improve the result (4-10). 


\section{INTERACTION OF THE LASER FIELD WITH THE ACTIVE MEDIUM (GENERAL CASE)}

The derivation of the expression for the polarization response in Chapter 3 was restricted to the case of (i) atomic transitions of the $j=1 \leftrightarrow j=0$ type; (ii) a single-mode electric field and (iii) no externally applied fields. For completeness, we will derive a similar expression for more general cases in this chapter. The procedure that will be followed is in fact equivalent to that of Sargent et al. [40] for the multi-mode case and that of Van Haeringen [39] for the single-mode case. It will include all types of atomic transitions $j_{a} \rightarrow_{b}$ for which $j_{a}-j_{b}=0,1$ or -1 . i.e., all cases allowing for electric-dipole transitions. The magnetic sublevels may be split up in energy due to the presence of an externally applied magnetic field $\underline{\mathrm{H}}$. Furthermore, an $\mathrm{N}$-mode electric field will be considered, where $N$ is a finite, positive integer.

Inevitably, such a derivation is lengthy and cumbersome, involving expressions which give little physical insight. In order to reduce the complexity of the resulting expressions somewhat, equally large Zeeman splittings of both atomic levels involved will be assumed. Furthermore, simplified relaxation equations will be used, i.e., unlike the treatment in Chapter 3, no difference in relaxation of the various multipole moments within one multiplet will be taken into account. The justification for this will be given in Section 5.1.

Differential equations for the density-matrix elements will be derived in Section 5.1. In Section 5.2 a solution of these equations will be obtained to the third order in the electric-field amplitudes. The expression for the polarization response will be derived in Section 5.3. 


\section{1 derivation of the equations}

Let $a$ and $b$ be the two energy levels involved in the transition under consideration, with energies $\hbar \Omega_{a}>\hbar \Omega_{b}$ and total-angular-momentum quantum numbers $j_{a}$ and $j_{b}$, respectively. We suppose that electric-dipole transitions between $\mathrm{a}$ and $\mathrm{b}$ are allowed, i.e., $j_{\mathrm{a}}-j_{\mathrm{b}}=0,1$ or -1 . Let all atoms be subject to a uniform magnetic field $\underline{H}$. The degenerate levels a and $\mathrm{b}$ will then be split up into magnetic sublevels. Let $\underline{\hat{x}}, \underline{\hat{y}}$ and $\underline{\hat{z}}$ be unit vectors of a right-handed cartesian coordinate system with $\underline{\hat{z}}=\underline{H} / H$. The wave functions belonging to the magnetic subleve1s are denoted by

$$
\left\{\Psi_{j_{\mathrm{a}}}^{\mathrm{a}}, \Psi_{j_{\mathrm{a}}-1}^{\mathrm{a}}, \cdots, \Psi_{-j_{\mathrm{a}}}^{\mathrm{a}} ; \Psi_{j_{\mathrm{b}}}^{\mathrm{b}}, \Psi_{j_{\mathrm{b}}-1}^{\mathrm{b}}, \ldots, \Psi_{-j_{\mathrm{b}}}^{\mathrm{b}}\right\}
$$

With respect to this base, the undisturbed hamiltonian can be represented by

$$
H_{0}=\left(\begin{array}{cc}
H_{0}^{\mathrm{aa}} & 0 \\
0 & H_{0}^{\mathrm{bb}}
\end{array}\right) \text {, }
$$

where

$$
\left(H_{0}^{\alpha \alpha}\right)_{m m^{\prime}}=\delta_{m m^{\prime}} \hbar\left(\Omega_{\alpha}+m v\right) .
$$

Here, $m$ and $m^{\prime}$ assume the values $j_{\alpha}, j_{\alpha-1}, \ldots,-j_{\alpha}$, where $\alpha=a$ or $b$, respectively, while the Zeeman-splitting frequency $\nu$ is given by

$$
\nu=\hbar^{-1} g m_{B} \mathrm{H}
$$

$m_{\mathrm{B}}$ being the Bohr magneton and $g$ the Landé factor, assumed to be equal for the two multiplets.

In the electric-dipole approximation adopted here, an electric field $\underline{E}$ couples to the electric-dipole operator $\underline{\mu}$ of the atom. We will assume that the electric field is of the following form: 


$$
\begin{aligned}
\underline{\mathrm{E}}(\underline{\underline{r}}, t)= & \frac{1}{2} \sum_{n=n_{1}+1}^{n_{1}+N}\left\{E_{1}(n, t) \frac{\frac{\hat{x}-i \underline{\hat{y}}}{\sqrt{2}}+E_{-1}(n, t) \frac{\frac{\hat{x}}{-i \hat{y}}}{\sqrt{2}}}{}\right. \\
& \left.+E_{0}(n, t) \underline{\hat{z}}\right\}^{-i \omega_{n} t} \sin \left(\underline{k}_{n} \cdot \underline{r}\right)+c \cdot c .,
\end{aligned}
$$

where $\omega_{n}=n \pi c / L$; the $\underline{k}_{n}$ all have the same direction and $n_{1}$ is a large positive integer, usually of order $10^{6}$. The electric field represented by $(5-5)$ corresponds to the excitation of $N$ successive longitudinal modes with weakly time-dependent, circular amplitudes $E_{1}(n, t), E_{-1}(n, t)$ and $E_{0}(n, t)$, respectively. The dipole-moment operator $\underline{\mu}$ is accordingly represented as

$$
\underline{\mu}=\mu_{1} \frac{\hat{\underline{x}}-i \hat{\underline{y}}}{\sqrt{2}}+\mu_{-1} \frac{\frac{\hat{x}}{}+i \underline{\hat{y}}}{\sqrt{2}}+\mu_{0} \hat{\underline{z}},
$$

where, for $l=0, \pm 1$,

$$
\mu_{2}=\left(\begin{array}{cc}
0 & \mu_{2}^{a b} \\
\mu_{2} a & 0
\end{array}\right)
$$

The matrix elements of $\mu_{2}$ are given by [62]

$$
\begin{aligned}
& \left(\mu_{\imath}^{\mathrm{ab}}\right)_{m m^{\prime}}=\delta_{m^{\prime}, m-l} p_{m, m-l} \\
& \left(\mu_{\imath}^{\mathrm{ba}}\right)_{m m^{\prime}}=\delta_{m^{\prime}, m-l} p_{m-\imath, m},
\end{aligned}
$$

where, for the various types of transitions $j_{a} \rightarrow j_{b}$,

$$
P_{m, m \pm 1}=\left\{\begin{array}{cc} 
\pm\{(j \mp m)(j \mp m-1)\}^{\frac{1}{2}} p, & j \rightarrow j-1 ; \\
\mp\{(j \pm m)(j \pm m+1)\}^{\frac{1}{2}} p, & j-1 \rightarrow j \\
\{(j \mp m)(j \pm m+1)\}^{\frac{1}{2}} p, & j \rightarrow j
\end{array}\right.
$$

and 


$$
P_{m, m}=\left\{\begin{array} { l } 
{ \{ 2 ( j + m ) ( j - m ) \} ^ { \frac { 1 } { 2 } } p , } \\
{ \sqrt { } 2 m p , }
\end{array} \quad \left\{\begin{array}{rl}
j & \rightarrow j-1 ; \\
j \rightarrow j
\end{array} ; .\right.\right.
$$

In (5-9) and (5-10) $p$ is a reduced matrix element related to the transition under consideration. Its value is independent of the magnetic quantum number $m$. For the $1 \rightarrow 0$ transition $p$ is given by (3-13); similar expressions can be written down for other transitions.

The perturbing hamiltonian reads

$$
\begin{aligned}
H_{i}=-\frac{1}{2} \sum_{l=0, \pm 1} \sum_{n=n_{1}+1}^{n+N} \mu_{2}\left\{E_{-l}(n, t) e^{-i \omega_{n} t}\right. \\
\left.+E_{\eta}^{*}(n, t) e^{i \omega_{n} t}\right\} \sin \left(\underline{k}_{n} \cdot \underline{r}\right) .
\end{aligned}
$$

The $\underline{r}$ dependent part in $H_{i}$, i.e., $\sin (\underline{k} \cdot \underline{r})$, can be taken to be constant over the dimensions of the atom. The matrix elements of $H_{i}$ are then directly related to the matrix elements of the $\mu_{\eta}$.

Let $\rho(\underline{r}, \underline{v}, t)$ be the density matrix for an atom having a position vector $\underline{r}$ and a velocity vector $\underline{v}$ at time $t$. The expectation value of the dipole moment for this atom is given by

$$
\langle\underline{\mu}(\underline{r}, \underline{v}, t)>=\operatorname{Tr}\{\rho(\underline{r}, \underline{v}, t) \underline{\mu}\} .
$$

The polarization $\underline{P}(\underline{p}, t)$ is then given by

$$
\underline{P}(\underline{r}, t)=d \int \underline{d} \underline{v} \Phi(\underline{v})<\underline{\mu}(\underline{r}, \underline{v}, t)>,
$$

where $d$ is the density of atoms and $\Phi(\underline{v})$ the normalized velocitydistribution function. The density matrix satisfies the equation of motion

$$
\partial_{t}^{v} \rho(\underline{r}, \underline{v}, t)=\frac{1}{i \hbar}\left[H_{0}+H_{i}, \rho(\underline{r}, \underline{v}, t)\right]+\left.\partial_{t} \rho(\underline{r}, \underline{v}, t)\right|_{\text {relax }},
$$

where $\partial_{t}^{2}$ is given by (3-34) and $\left.\partial_{t} \rho\right|_{\text {relax }}$ is the contribution of relaxations to the time derivative of $\rho$. Representing $\rho$ as 
$\rho=\left(\begin{array}{ll}\rho^{a a} & \rho^{a b} \\ \rho^{a b t} & \rho^{b b}\end{array}\right)$,

we will assume the following simple relaxation equations:

$$
\begin{aligned}
& \left.\partial_{t} \rho^{\alpha \alpha}\right|_{\text {relax }}=-\lambda_{\alpha}\left(\rho^{\alpha \alpha}-\overline{\rho^{\alpha \alpha}}\right), \quad(\alpha=a, b) ; \\
& \left.\partial_{t} \rho^{a b}\right|_{\text {relax }}=-\lambda \rho^{a b},
\end{aligned}
$$

where $\lambda_{a}$ and $\lambda_{b}$ are the global relaxation rates of the upper and lower level, respectively, and $\lambda$ is to be identified with the line width of the transition. Furthermore, $\overline{\rho^{\alpha \alpha}}(\alpha=a, b)$ denote the equilibrium density matrices of the upper and lower level, respectively, i.e., the density matrices corresponding to the state that will be reached in the absence of the perturbation $H_{i}$. When describing the relaxation process by (5-16) and (5-17), we neglect the possible occurrence of different relaxation rates for the various multipole moments of $\rho^{a a}, \rho^{b b}$ and $\rho^{a b}$. The justification for this can best be given by referring to (3-69), valid in the $j=1 \rightarrow j=0$ transition case. It is observed that the right-hand side of (3-69) would be equal to zero if $\lambda_{J}=\lambda_{Q}$, that is, the time evolution of the ellipticity depends heavily on differences in $\lambda_{J}$ and $\lambda_{Q}$ (see Section 4.1). Therefore, in order to explain a medium-induced anisotropy for this transition, it was essential to include a priori the occurrence of different relaxation rates within one multiplet. A similar reasoning holds for $0 \rightarrow 1$ and $1 \rightarrow 1$ transitions. For all other transitions, however, it appears that the equivalent of (3-69) has terms in the right-hand side not present in the three above-mentioned transition cases. These terms largely exceed in magnitude the terms due to the differences in multipole relaxation rates. Therefore, the omission of the differences in the relaxation rates of the respective multipole moments will not lead to qualitatively different results, as long as only the polarization behaviour is considered.

As in Chapter 3, we will assume that the pumping process is isotropic and homogeneous, while the equilibrium density matrix $\bar{\rho}$ is independent of 
the velocity of the atom. We then have

$$
\bar{\rho}=\left(\begin{array}{cc}
\overline{\rho^{\mathrm{aa}}} & 0 \\
0 & \overline{\rho^{\mathrm{bb}}}
\end{array}\right)=\left(\begin{array}{cc}
\overline{\mathrm{N}}_{\mathrm{a}} 1_{\mathrm{a}} & 0 \\
0 & \overline{\mathrm{N}}_{\mathrm{b}} 1_{\mathrm{b}}
\end{array}\right)
$$

where $\overline{\mathrm{N}}_{\mathrm{a}}$ and $\overline{\mathrm{N}}_{\mathrm{b}}$ are positive numbers related to the populations of the upper and lower level, respectively; $1_{a}$ and $1_{b}$ are the respective unit matrices. By introducing the deviation $R$ of $\rho$ from equilibrium, i.e.,

$$
\rho(\underline{r}, \underline{v}, t)=\bar{\rho}+R(\underline{r}, \underline{v}, t),
$$

we can rewrite (5-14), by using (5-16) and (5-17), and by evaluating the commutator $\left[H_{0}, 0\right]$, in the following form:

$$
\left\{\partial_{t}^{v}+i\left(m-m^{\prime}\right) v+\lambda_{\alpha}\right\} R_{m m^{\prime}}^{\alpha \alpha}=\frac{1}{i \hbar}\left[H_{i}, \bar{\rho}+R\right]_{m m^{\prime}}^{\alpha \alpha} .
$$

for $\alpha=a$ and $\alpha=b$, respectively, and

$$
\left\{\partial_{t}^{v}+i \Omega+i\left(m-m^{\prime}\right) v+\lambda\right\} R_{m m^{\prime}}^{a b}=\frac{1}{i \hbar}\left[H_{i}, \bar{\rho}+R\right]_{m m^{\prime}}^{a b} .
$$

These equations have to be solved for $H_{i}$ given by (5-11), as will be done in the next section.

\section{2 third-order solution of the equations}

In order to solve (5-20) and (5-21) we will follow an iterative procedure similar to that in Section 3.2. An expression for $R^{a b}$ will be derived to the third order in the driving field. The first-order contribution to $R$ can be obtained by taking $R=0$ in the right-hand sides of $(5-20)$ and (5-21), and by substitution of (5-11) for $H^{\prime}$. The result is 


$$
R^{\alpha \alpha(1)}=0
$$

for $\alpha=a$ or $b$, and

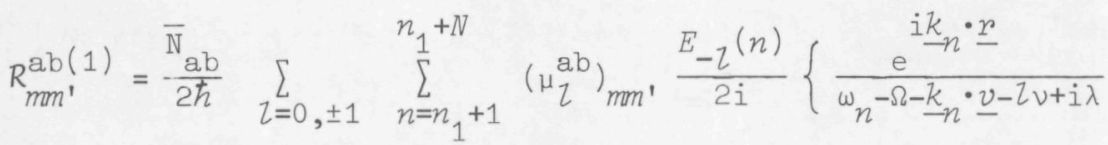

$$
\begin{aligned}
& \left.-\frac{e^{-\underline{i k} \cdot n} \cdot \underline{r}}{\omega_{n}-\Omega+\underline{k}_{n} \cdot \underline{v}-2 v+i \lambda}\right\} e^{-i \omega_{n} t}
\end{aligned}
$$

where $\bar{N}_{a b}$ is the population difference,

$$
\bar{N}_{a b}=\bar{N}_{a}-\bar{N}_{b} \text {. }
$$

In (5-23) all nonresonant terms, i.e., terms with $\omega_{n}+\Omega$ in the denominator, have been omitted, while the approximations used in the derivation of (3-53) have also been applied. The expression for $R^{\text {ba(1) }}$ can be derived from (5-23), by hermitian conjugation, i.e.,

$$
R^{\mathrm{ba}(1)}=\left\{R^{\mathrm{ab}(1)}\right\}^{\dagger} .
$$

In the second iteration step we substitute $R=R^{(1)}$ in the right-hand sides of (5-20) and (5-21). Then, after neglecting nonresonant terms, we obtain

$$
\begin{aligned}
& R_{m m^{\prime}}^{\mathrm{aa}(2)}=\frac{\overline{\mathrm{N}}_{\mathrm{ab}}}{16 \hbar^{2}} \sum_{\substack{\eta^{\prime}, \eta^{\prime}=\\
0, \pm 1}} \sum_{\substack{n_{1}, n^{\prime}=\\
n_{1}+1}}^{n_{1}+N}\left(\mu_{\eta}, \mu_{\eta}\right)_{m m^{\prime}}^{\mathrm{aa}} E_{-\eta^{\prime}}(n) E_{\eta^{\prime}}^{*},\left(n^{\prime}\right) \mathrm{e}^{-i\left(\omega_{n^{-}}-\omega_{n^{\prime}}\right) t} \\
& \times\left\{G_{1}\left(\underline{k}_{n}, \underline{k}_{n^{\prime}}\right)+G_{1}\left(-\underline{k}_{n},-\underline{k}_{n^{\prime}}\right)-G_{1}\left(-\underline{k}_{n}, \underline{k}_{n},\right)-G_{1}\left(\underline{k}_{n},-\underline{k}_{n^{\prime}}\right)\right\} \text {, }
\end{aligned}
$$

where

$$
\begin{aligned}
G_{1}\left(\underline{k}, \underline{k}^{\prime}\right) & =\frac{e^{i\left(\underline{k}+\underline{k}^{\prime}\right) \cdot \underline{r}}}{-\left(\omega_{n}-\omega_{n^{\prime}}\right)+\left(\underline{k}+\underline{k}^{\prime}\right) \cdot \underline{v}+\left(Z+l^{\prime}\right) \nu-i \lambda} a \\
& \times\left\{\frac{1}{-\left(\omega_{n^{\prime}}-\Omega\right)-\underline{k}^{\prime} \cdot \underline{v}-l^{\prime} v+\bar{i} \lambda}+\frac{1}{\omega_{n}-\Omega-\underline{k} \cdot \underline{v}-l v+i \lambda}\right\} .
\end{aligned}
$$


The expression for $R^{\mathrm{bb}(2)}$ can be obtained from (5-26) by multip1ying the right-hand side by -1 , replacing a by $b$, and turning around the order of $\mu_{Z}$, and $\mu_{\eta}$. There are no second-order contributions to $R^{a b}$.

The third-order contribution to $R^{a b}$ can now be derived from (5-21), by substitution of (5-26). Again neglecting nonresonant terms, we obtain the result

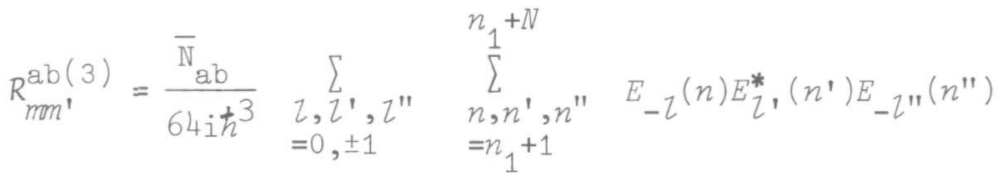

$$
\begin{aligned}
& e^{-i\left(\omega_{n^{\prime \prime}}+\omega_{n}-\omega_{n}\right) t}\left\{G_{2}\left(-\underline{k}_{-n}, \underline{k}_{n}, \underline{k}_{n^{\prime \prime}}\right)+G_{2}\left(\underline{k}_{n},-\underline{k}_{n^{\prime}}, \underline{k}_{n^{\prime \prime}}\right)\right. \\
& +G_{2}\left(\underline{k}_{n}, \underline{k}_{n},,-\underline{k}_{n}{ }^{\prime}\right)+G_{2}\left(\underline{k}_{n}, \underline{k}_{n},, \underline{k}_{n}\right)-G_{2}\left(\underline{k}_{n},-\underline{k}_{n},,-\underline{k}_{n}\right) \\
& \left.-G_{2}\left(-\underline{k}_{n}, \underline{k}_{n},,-\underline{k}_{n}^{\prime \prime}\right)-G_{2}\left(-\underline{k}_{n},-\underline{k}_{n},, \underline{k}_{n}{ }^{\prime}\right)-G_{2}\left(-\underline{k}_{n},-\underline{k}_{n},,-\underline{k}_{n}^{\prime \prime}\right)\right\} \text {, }
\end{aligned}
$$

where

$$
\begin{aligned}
& G_{2}\left(\underline{k}, \underline{k}^{\prime}, \underline{k}^{\prime \prime}\right)=\frac{\mathrm{e}^{i\left(\underline{k}+\underline{k}^{\prime}+\underline{k}^{\prime \prime}\right) \cdot \underline{p}}}{\omega_{n^{\prime \prime}}+\omega_{n}-\omega_{n^{\prime}}-\Omega-\left(\underline{k}^{\prime} \underline{k}^{\prime}+\underline{k}^{\prime \prime}\right) \cdot \underline{v}-\left(\eta+l^{\prime}+Z^{\prime \prime}\right) v+i \lambda}
\end{aligned}
$$

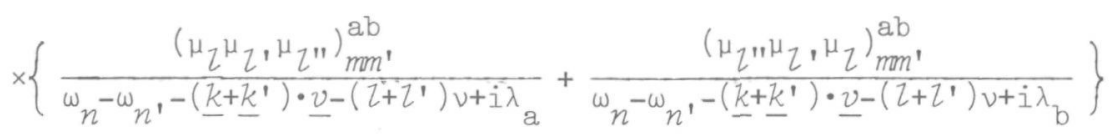

$$
\begin{aligned}
& \times\left\{\frac{1}{-\left(\omega_{n},-\Omega\right)-\underline{k^{\prime}} \cdot \underline{v}-Z^{\prime} v+i \lambda}+\frac{1}{\omega_{n}-\Omega-\underline{k} \cdot \underline{v}-2 v+i \lambda}\right\},
\end{aligned}
$$

\section{3 third-order expression for the polarization response}

According to (5-12) and (5-13), the circular components of the polarization are given by $(\tau=0, \pm 1)$

$$
\mathrm{P}_{\mathcal{Z}}(\underline{r}, t)=d \int \mathrm{d} \underline{v} \Phi(\underline{v}) \operatorname{Tr}\left\{\rho(\underline{r}, \underline{v}, t) \mu_{\eta}\right\} .
$$


The trace in (5-30) can be written as

$$
\operatorname{Tr}\left(\rho \mu_{\eta}\right)=\operatorname{Tr}\left\{\left\{R^{a b(1)}+R^{a b(3)}\right\} \mu_{\eta}^{b a}\right\}+c \cdot c .
$$

From now on, we will assume that $\Phi$ is a symmetric function of $\underline{v}$. The firstorder contribution to $\mathrm{P}_{2}$ can be calculated by using (5-23), which leads to

$$
\begin{aligned}
\mathrm{P}_{\eta}^{(1)}(\underline{\underline{r}}, t)= & \frac{\overline{\mathrm{N}}}{2 \hbar} \sum_{\eta^{\prime}=0, \pm 1} \sum_{n=n_{1}+1}^{n_{1}+N} \operatorname{Tr}\left(\mu_{\eta}, \mu_{\eta}\right)^{\text {aa }} E_{-\eta},(n) \mathrm{e}^{-i \omega_{n} t} \\
& \times \sin (\underline{\underline{k}} \cdot \underline{\underline{r}}) \int \frac{\mathrm{d} \underline{\underline{v}} \Phi(\underline{v})}{\omega_{n}^{-\Omega+\underline{k}_{n} \cdot \underline{v}-\eta^{\prime} v+i \lambda}}+c \cdot c .
\end{aligned}
$$

where

$$
\overline{\mathrm{N}}=d \overline{\mathrm{N}}_{\mathrm{ab}} .
$$

For the third-order contribution we obtain, using (5-28) and (5-29),

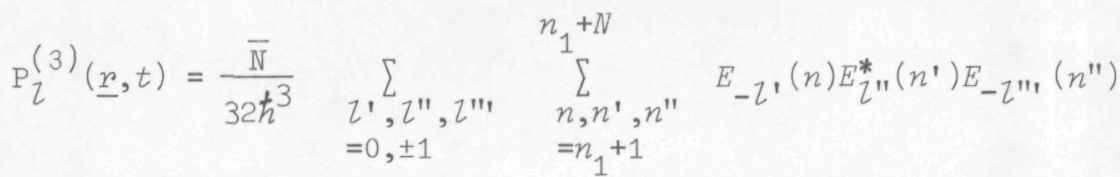

$$
\begin{aligned}
& x^{-i\left(\omega_{n}^{\prime \prime}+\omega_{n}-\omega_{n}^{\prime}\right) t}\left\{G_{3}\left(-\underline{k}_{n}, \underline{k}_{n^{\prime}}, \underline{k}_{n^{\prime \prime}}\right)+G_{3}\left(\underline{k}_{n},-\underline{k}_{n}, \underline{k}_{n^{\prime \prime}}\right)\right. \\
& \left.+G_{3}\left(\underline{k}_{n}, \underline{k}_{n},,-\underline{k}_{n}{ }^{\prime \prime}\right)-G_{3}\left(\underline{k}_{n}, \underline{k}_{n^{\prime}}, \underline{k}_{n^{\prime \prime}}\right)\right\}+c . c .,
\end{aligned}
$$

where

$$
\begin{aligned}
& G_{3}\left(\underline{k}, \underline{k}^{\prime}, \underline{k}^{\prime \prime}\right)=\int \underline{\mathrm{v}} \Phi(\underline{v})\left\{\frac{\sin \left\{\left(\underline{k}+\underline{k}^{\prime}+\underline{k}^{\prime \prime}\right) \cdot \underline{r}\right\}}{\omega_{n^{\prime \prime}}+\omega_{n}{ }^{-\omega_{n}{ }^{\prime}}-\left(\underline{k}+\underline{k}^{\prime}+\underline{k}^{\prime \prime}\right) \cdot \underline{v}-\left(Z^{\prime}+Z^{\prime \prime}+Z^{\prime \prime \prime}\right) v+i \lambda}\right.
\end{aligned}
$$

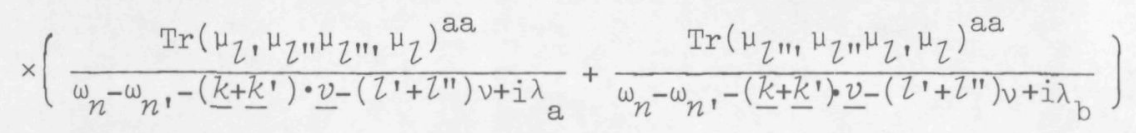

$$
\begin{aligned}
& \left.\times\left(\frac{1}{-\left(\omega_{n}{ }^{-\Omega}\right)-\underline{k}^{\prime} \cdot \underline{v}-Z^{\prime \prime} v+i \lambda}+\frac{1}{\omega_{n}-\Omega-\underline{k} \cdot \underline{v}-L^{\prime} v+i \lambda}\right)\right\} \text {. }
\end{aligned}
$$


Let us first discuss the traces present in (5-32) and (5-34). It can easily be seen, when we use, for instance, the matrix representation given by $(5-7)-(5-10)$, that

$$
\left.\begin{array}{ll}
\operatorname{Tr}\left(\mu_{\eta}, \mu_{\eta}\right)^{\text {aa }}=0, & \text { if } \tau+\eta^{\prime} \neq 0 ; \\
\operatorname{Tr}\left(\mu_{\eta}, \mu_{\eta \prime} \mu^{\prime \prime \prime} \mu_{\eta}\right)^{\text {aa }}=0, & \text { if } \eta^{\prime}+\eta^{\prime \prime}+\eta^{\prime \prime}+\tau \neq 0 .
\end{array}\right\}
$$

If we use the explicit expressions (5-9) and (5-10) for the matrix elements, the nonvanishing traces can easily be calculated. We find for the various types of transitions

$$
F_{1} \equiv p^{-2} \operatorname{Tr}\left(\mu_{\tau^{\mu}-\imath}\right)^{\text {aa }}= \begin{cases}(2 / 3)(j+1)(2 j+1)(2 j+3), & j \leftrightarrow j+1 ; \\ (2 / 3) j(j+1)(2 j+1), & j \rightarrow j,\end{cases}
$$

for $\ell=0, \pm 1$, and,

$$
\begin{aligned}
& \left.F_{2} \equiv p^{-4 \operatorname{Tr}\left(\mu_{1}{ }^{\mu}-1^{\mu}{ }_{1}{ }^{\mu}-1\right.}\right)^{\mathrm{aa}}= \begin{cases}(2 / 5) F_{1}\left(6 j^{2}+12 j+5\right), & j \leftrightarrow j+1 ; \\
(2 / 5) F_{1}\left(2 j^{2}+2 j+1\right), & j \rightarrow j ;\end{cases} \\
& F_{3} \equiv p^{-4} \operatorname{Tr}\left(\mu_{0} \mu_{0} \mu_{0} \mu_{0}\right)^{\mathrm{aa}}= \begin{cases}(2 / 5) F_{1}\left(4 j^{2}+8 j+5\right), & j \leftrightarrow j+1 ; \\
(2 / 5) F_{1}\left(3 j^{2}+3 j-1\right), & j \rightarrow j ;\end{cases} \\
& F_{4} \equiv p^{-4} \operatorname{Tr}\left(\mu_{1} \mu_{0} \mu_{0} \mu_{-1}\right)^{\mathrm{aa}}= \begin{cases}(1 / 5) F_{1} j(6 j+7), & j \rightarrow j+1 ; \\
(1 / 5) F_{1}(j+2)(6 j+5), & j+1 \rightarrow j ; \\
(1 / 2) F_{1}, & j \rightarrow j .\end{cases}
\end{aligned}
$$

All other nonvanishing traces can be expressed in terms of $F_{2}, F_{3}$ and $F_{4}$ :

$$
\left.\begin{array}{rl}
\operatorname{Tr}\left(\mu_{-1} \mu_{1} \mu_{-1} \mu_{1}\right)^{\text {aa }} & =F_{2} p^{4} ; \operatorname{Tr}\left(\mu_{-1} \mu_{0} \mu_{0} \mu_{1}\right)^{\text {aa }}=F_{4} p^{4} ; \\
\operatorname{Tr}\left(\mu_{1} \mu_{-1} \mu_{0} \mu_{0}\right)^{\text {aa }}= & \operatorname{Tr}\left(\mu_{0} \mu_{0} \mu_{1} \mu_{-1}\right)^{\text {aa }}=\operatorname{Tr}\left(\mu_{0} \mu_{0} \mu_{-1} \mu_{1}\right)^{\text {aa }} \\
= & \operatorname{Tr}\left(\mu_{-1} \mu_{1} \mu_{0} \mu_{0}\right)^{\text {aa }}=\left(F_{2}-F_{4}\right) p^{4} ; \\
\operatorname{Tr}\left(\mu_{1} \mu_{1} \mu_{-1} \mu_{-1}\right)^{\text {aa }}=\operatorname{Tr}\left(\mu_{-1} \mu_{-1} \mu_{1} \mu_{1}\right)^{\text {aa }}=\left(F_{3}+F_{4}-F_{2}\right) p^{4} ;
\end{array}\right\}
$$




$$
\begin{aligned}
\operatorname{Tr}\left(\mu_{1} \mu_{-1} \mu_{-1} \mu_{1}\right)^{\mathrm{aa}} & =\operatorname{Tr}\left(\mu_{-1} \mu_{1} \mu_{1} \mu_{-1}\right)^{\mathrm{aa}}=\left(F_{3}-F_{4}\right) p^{4} \\
\operatorname{Tr}\left(\mu_{1} \mu_{0} \mu_{-1} \mu_{0}\right)^{\mathrm{aa}} & =\operatorname{Tr}\left(\mu_{0} \mu_{-1} \mu_{0} \mu_{1}\right)^{\mathrm{aa}}=\operatorname{Tr}\left(\mu_{0} \mu_{1} \mu_{0} \mu_{-1}\right)^{\mathrm{aa}} \\
& =\operatorname{Tr}\left(\mu_{-1} \mu_{0} \mu_{1} \mu_{0}\right)^{\mathrm{aa}}=\left(F_{3}-F_{2}\right) p^{4} .
\end{aligned}
$$

Next, we will discuss the velocity integrals present in (5-32) and (5-34). The integral occurring in the first-order expression (5-32) reads

$$
\int \frac{\mathrm{d} \underline{\underline{v}} \Phi(\underline{v})}{w+\underline{k} \cdot \underline{v}+i \lambda}
$$

where $w=\omega_{n}-\Omega-\eta^{\prime} \nu$. In practice, the successive $k_{n}$ to be considered in (5-42) are all lying very close to each other, i.e., $\left|\underline{k}_{n}-\underline{k}_{n}\right| \sim 10^{-6} k$, where $k=\Omega / c$ and where $\Omega$ is the central atomic transition frequency. A good approximation of (5-42) is therefore obtained by replacing all $\underline{k}_{n}$ by $\underline{k}$, i.e., by taking

$$
\int \frac{d \underline{v} \Phi(\underline{v})}{w+\underline{k} \cdot \underline{v} \underline{v}+i \lambda} \simeq \int \frac{d \underline{v} \Phi(\underline{v})}{w+\underline{k} \cdot \underline{v}+i \lambda} \equiv-I_{0}(w)
$$

One of the integrals in $(5-35)$ is of the form

$$
\int d \underline{v} \Phi(\underline{v}) \frac{1}{\left\{w_{1}-\left(\underline{k}_{n}{ }^{\prime}-\underline{k}_{n}+\underline{k}_{n}\right) \cdot \underline{v}^{+i \lambda}\right\}\left\{w_{2}+\left(\underline{k}_{n}-\underline{k}_{n}\right) \cdot \underline{v}^{+i \lambda} \lambda_{\alpha}\right\}\left\{w_{3}-\underline{k}_{n} \cdot v+i \lambda\right\}} .
$$

The second term of the denominator in (5-44) is a slowly varying function of $\underline{v}$, as compared to the other terms, and can be replaced by $\left(\omega_{2}+i \lambda_{\alpha}\right)^{-1}$. Then, the remaining integral can be expressed in terms of integrals of the kind (5-42). The result is that expression (5-44) can be consistently approximated by

$$
\frac{1}{\left(w_{2}+i \lambda_{\alpha}\right)\left(w_{1}-w_{3}\right)}\left\{I_{0}\left(w_{1}\right)-I_{0}\left(w_{3}\right)\right\}=\frac{1}{w_{2}+i \lambda_{\alpha}} I_{2}\left(w_{1}, w_{3}\right)
$$


where

$$
I_{2}\left(w_{1}, w_{3}\right)=\frac{I_{0}\left(w_{1}\right)-I_{0}\left(w_{3}\right)}{w_{1}-w_{3}} .
$$

Other integrals in (5-35) can also be approximated by more simple expressions by neglecting the differences $\underline{k}_{n}-\underline{k}_{n}$, with respect to $\underline{k}$. This leads to the following five functions, in terms of which each integral in (5-35) can be expressed:

$$
\begin{aligned}
& I_{0}(w)=-\int \frac{\mathrm{d} \underline{v} \Phi(\underline{v})}{w-\underline{k} \cdot \underline{v}+i \lambda} \\
& I_{1}\left(w_{1}, w_{2}\right)=\int \frac{\mathrm{d} \underline{v} \Phi(\underline{v})}{\left(w_{1}-\underline{k} \cdot \underline{v}+i \lambda\right)\left(w_{2}+\underline{k} \cdot \underline{v}+i \lambda\right)}=-\frac{I_{0}\left(w_{1}\right)+I_{0}\left(w_{2}\right)}{w_{1}+w_{2}+2 i \lambda} ; \\
& I_{2}\left(w_{1}, w_{2}\right)=\int \frac{d \underline{v} \Phi(\underline{v})}{\left(w_{1}-\underline{k} \cdot \underline{v}+i \lambda\right)\left(w_{2}-\underline{k} \cdot \underline{v}+i \lambda\right)}= \begin{cases}\frac{I_{0}\left(w_{1}\right)-I_{0}\left(w_{2}\right)}{w_{1}-w_{2}}, & \left(w_{1} \neq w_{2}\right) ; \\
\partial_{w_{1}} I_{0}\left(w_{1}\right), & \left(w_{1}=w_{2}\right) ;\end{cases} \\
& I_{3}^{\alpha}\left(w_{1}, w_{2}, w_{3}\right)=\int \frac{d \underline{v} \Phi(\underline{v})}{\left(w_{1}-\underline{k} \cdot \underline{v}+i \lambda\right)\left(w_{2}-\underline{2} \underline{k} \cdot \underline{v}+i \lambda_{\alpha}\right)\left(w_{3}-\underline{k} \cdot \underline{v}+i \lambda\right)}, \quad(\alpha=a, b) ; \\
& I_{4}^{\alpha}\left(w_{1}, w_{2}, w_{3}\right)=\int \frac{d \underline{v} \Phi(\underline{v})}{\left(w_{1}-\underline{k} \cdot \underline{v}+i \lambda\right)\left(w_{2}-2 \underline{k} \cdot \underline{v}+i \lambda \alpha\right)\left(w_{3}-3 \underline{k} \cdot \underline{v}+i \lambda\right)}, \quad(\alpha=a, b) .
\end{aligned}
$$

A further approximation concerns the actual calculation of the integrals (5-47). First, we will substitute the Maxwell distribution function (3-74) for $\Phi(\underline{v})$, and will introduce some related dimensionless quantities. If $\lambda_{\mathrm{d}}$ is the Doppler width, i.e.,

$$
\lambda_{\mathrm{d}}=k \bar{v},
$$

where $\bar{v}$ is the r.m.s. velocity, then we define 


$$
\begin{array}{rlrl}
s_{n} & =\left(\omega_{n}-\Omega\right) / \lambda_{d} ; & & \text { (detuning parameter of mode } n \text { ) } \\
\lambda=\lambda / \lambda_{d} ; & & \text { (transition line width parameter) } \\
\lambda_{\alpha}=\lambda_{\alpha} / \lambda_{d},(\alpha=a, b) & ; \text { (natural line width parameter) } \\
h=\nu / \lambda_{d} . & & \text { (magnetic field parameter) }
\end{array}
$$

Dimensionless functions are introduced by defining

$$
\left.\begin{array}{l}
J_{0}\left(w / \lambda_{d}\right)=\lambda_{d} I_{0}(w) ; \\
J_{q}\left(w_{1} / \lambda_{d}, w_{2} / \lambda_{d}\right)=\lambda_{d}^{2} I_{q}\left(w_{1}, w_{2}\right), \quad(q=1,2) ; \\
J_{q}^{\alpha}\left(w_{1} / \lambda_{d}, w_{2} / \lambda_{d}, w_{3} / \lambda_{d}\right)=\lambda_{d}^{3} I_{q}^{\alpha}\left(w_{1}, w_{2}, w_{3}\right), \quad(q=3,4 \text { and } \alpha=a, b) .
\end{array}\right\}
$$

In all cases in which the transition line width parameter $\lambda \ll 1$, the calculation of the integrals can be considerably simplified, by using approximate expressions. In that case, of ten referred to as "Doppler limit", $J_{0}$ is given by [see also (3-75)]

$$
J_{0}(s)=2 e^{-s^{2}} \int_{0}^{s} d t e^{t^{2}}+i \sqrt{ } \pi e^{-s^{2}},
$$

while $J_{3}^{\alpha}$ and $J_{4}^{\alpha}$ are vanishingly small. Although a value of $\chi$ as 1 arge as 0.1 can certainly occur in practice, evaluation of the integrals in the Doppler limit will not affect the qualitative results to be obtained in the following chapters.

Using all these approximations in (5-32) and (5-35), we can write the expression for the polarization response as 


$$
\begin{aligned}
& \left(\Omega / \varepsilon_{0}\right) P_{\eta}(\underline{r}, t)=-\frac{1}{2} \frac{\Omega \bar{N} p^{2}}{\varepsilon_{0} \hbar \lambda_{d}} F_{1} \sum_{n=n_{1}+1}^{n_{1}+N} J_{0}\left(s_{n}+l \cdot h\right) E_{\eta}(n) e^{-i \omega_{n} t} \sin \left(\underline{k_{n}} \cdot \underline{r}\right)
\end{aligned}
$$

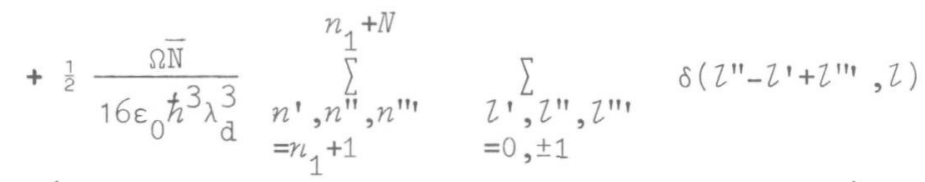

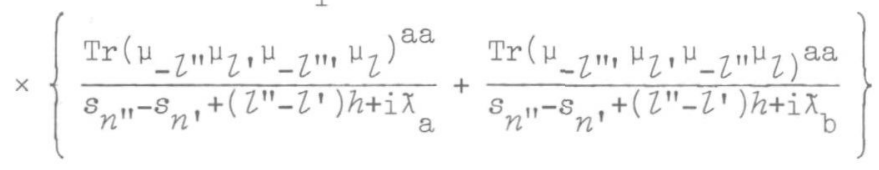

$$
\begin{aligned}
& \times\left\{\left\{J_{2}\left(s_{n " 1}+s_{n "},-s_{n,}+2 h,-s_{n},-l, h\right)+J_{1}\left(s_{n, "}+s_{n "}-s_{n,}+2 h, s_{n "}+Z " h\right)\right\}\right. \\
& \times \sin \left(\underline{k}_{n},-\underline{k}_{n "}+\underline{k}_{n "},\right) \cdot \underline{r} \\
& +\left\{J_{1}\left(s_{n " 1}+s_{n "}-s_{n,}+Z h,-s_{n},-\tau, h\right)+J_{2}\left(s_{n "},+s_{n "}-s_{n},+Z h, s_{n "}+\tau " h\right)\right\} \\
& \left.\times \sin \left(-\underline{k}_{n},+\underline{k}_{n},+\underline{k}_{n}, 1\right) \cdot \underline{r}\right\} \\
& \times E_{\eta_{1}}^{*}\left(n^{\prime}\right) E_{\eta^{\prime}}\left(n^{\prime \prime}\right) E_{\eta^{\prime \prime}}\left(n^{\prime \prime \prime}\right) e^{-i\left(\omega_{n^{\prime \prime}}+\omega_{n^{\prime \prime}}-\omega_{n}, t\right.}+\text { c.c. }
\end{aligned}
$$

In the second term in the right-hand side of (5-52) mode patterns are present, some of which are not contained in the original electric field (5-5). However, it is assumed that such terms do not contribute to the time evolution of the electric field in a laser. Therefore, instead of the full expression (5-52) we now consider its projection $\mathrm{P}_{l}^{\operatorname{proj}^{j}}(\underline{r}, t)$ on the originally assumed natura1-modes. We then find

$$
\begin{aligned}
& \left(\Omega / \varepsilon_{0}\right) \mathrm{P} \frac{\operatorname{proj}}{l}(\underline{r}, t)=-\frac{1}{2} \sum_{n=n_{1}+1}^{n_{1}+N} a_{\eta}^{n_{E}}(n) e^{-i \omega_{n} t} \sin (\underline{\underline{k}} \cdot \underline{\underline{r}})
\end{aligned}
$$

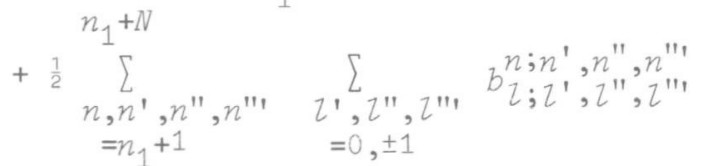

$$
\begin{aligned}
& \times E_{Z^{\prime}}^{*}\left(n^{\prime}\right) E_{Z^{\prime \prime}}\left(n^{\prime \prime}\right) E_{Z^{\prime \prime}}\left(n^{\prime \prime \prime}\right) \mathrm{e}^{-i\left(\omega^{\prime \prime \prime}+\omega_{n "}-\omega_{n^{\prime}}\right) t} \sin \left(\underline{k}_{n} \cdot \underline{\underline{r}}\right)+c \cdot c .
\end{aligned}
$$


where the $a$ and $b$ coefficients are given by

$$
\begin{aligned}
& a_{\eta}^{n}=\frac{\Omega \overline{\mathrm{N}} p^{2}}{\varepsilon_{0} \hbar \lambda_{\mathrm{d}}} F_{1} J_{0}\left(s_{n}+2 \eta_{2}\right)
\end{aligned}
$$

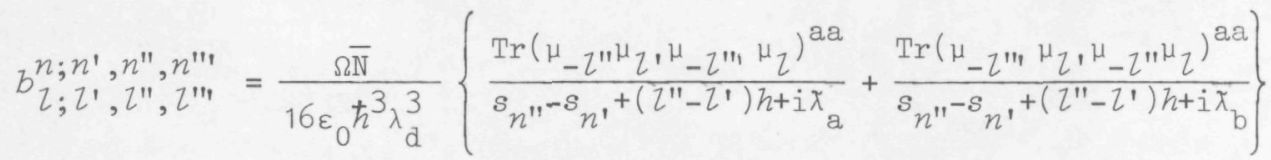

$$
\begin{aligned}
& \times\left\{\delta ( n ^ { \prime \prime \prime } - n ^ { \prime \prime } + n ^ { \prime } , n ) \left\{J_{2}\left(s_{n^{\prime \prime \prime}}+s_{n^{\prime \prime}}-s_{n^{\prime}}+2 h,-s_{n^{\prime}}-Z^{\prime} h\right)+\right.\right. \\
& \left.+J_{1}\left(s_{n^{\prime \prime}}+s_{n^{\prime \prime}}-s_{n^{\prime}}+2 h, s_{n^{\prime \prime}}+2 " h\right)\right\} \\
& +\delta\left(n^{\prime \prime \prime}+n^{\prime \prime}-n^{\prime}, n\right)\left\{\mathcal{J}_{1}\left(s_{n^{\prime \prime}}+s_{n^{\prime \prime}}-s_{n^{\prime}}+2 h,-s_{n^{\prime}}-2, h\right)\right. \\
& \left.\left.+J_{2}\left(s_{n^{\prime \prime}}+s_{n^{\prime \prime}}-s_{n^{\prime}}+2 h, s_{n^{\prime \prime}}+Z^{\prime \prime} h\right)\right\}\right\} \text {. }
\end{aligned}
$$

Inspection of (5-55) shows that the nonvanishing $b$ coefficients can be arranged in three groups, namely, those for which:

(a) $n^{\prime}=n^{\prime \prime}$ and $n^{\prime \prime \prime}=n$;

(b) $n^{\prime} \neq n^{\prime \prime}$ and $n^{\prime \prime \prime} \neq n$, while $n^{\prime \prime \prime}+n^{\prime \prime}-n^{\prime}=n$;

(c) $n^{\prime} \neq n^{\prime \prime}$ and $n^{\prime \prime \prime} \neq n$, while $n^{\prime \prime \prime}-n^{\prime \prime}+n^{\prime}=n$.

Note that groups (b) and (c) only occur if $N \geq 2$. Calculations of the $b$ coefficients show that the coefficients in groups (b) and (c) assume values that generally are two orders of magnitude smaller than those in group (a), at least for magnetic-field splittings which are small when compared to the mode spacing. The coefficients in group (c) give rise to terms in (5-53) with a space-time dependence not contained in the electricfield representation (5-5), i.e., they lead to terms in which $\underline{k}_{n}$ is combined with a frequency different from $\omega_{n}$. These terms are already small and it can moreover be shown by using the laser equation (2-26) that, due to the mismatch of wave number and frequency, these terms lead to a contribution to the time evolution of the electric-field amplitude that is even smaller by an additional factor equal to the ratio of the cavity line width and the mode spacing. Hence, in practical cases the coefficients in group (c) can be neglected. The coefficients in group 
can be neglected if both the line widths $\lambda_{a}$ and $\lambda_{b}$ and the magnetic-field parameter $|h|$ are small when compared to the mode spacing parameter $s_{n}-s_{n-1}$. Assuming the latter conditions to be satisfied, we finally arrive at the following expression for the (projected) polarization response:

$$
\mathrm{P}_{\eta}^{\operatorname{proj}}(\underline{\underline{r}}, t)=\frac{1}{2} \sum_{n=n_{1}+1}^{n_{1}+N} P_{\eta}(n) e^{-i \omega_{n} t} \sin (\underline{\underline{k}} \cdot \underline{r})+c \cdot c .,
$$

where

$$
\begin{aligned}
& \left(\Omega / \varepsilon_{0}\right) P_{\eta}(n)=-a_{\eta}^{n} E_{\eta}(n) \\
& { }^{n} \sum_{1}^{+N}, n^{\prime \prime}, n^{\prime \prime \prime} \\
& =n_{1}+1 \text {; } \\
& n=-n^{\prime}+n^{\prime \prime}+n^{\prime \prime} \\
& Z^{\prime}, Z^{\prime \prime}, Z^{\prime \prime} \\
& b_{Z ; Z !, Z ", Z n^{\prime \prime}}^{n ; n^{\prime}, n^{\prime \prime}, n^{\prime \prime}} E^{*},\left(n^{\prime}\right) E_{Z^{\prime \prime}}\left(n^{\prime \prime}\right) E_{Z^{\prime \prime}}\left(n^{\prime \prime \prime}\right) . \\
& =0, \pm 1 \text {; } \\
& Z=-Z{ }^{\prime}+Z "+Z " \prime
\end{aligned}
$$




\section{POLARIZATION EFFECTS IN SINGLE-MODE AND TWO-MODE GAS LASERS}

This chapter deals with the polarization effects in a gas laser for those cases in which one or two longitudinal modes are excited, while no external fields are applied. It is noted in this connection that the polarization behaviour of gas lasers in which more than one mode is excited has received much less attention than that of a laser operating in one single mode. Although it is for instance well known that successive longitudinal modes of a multi-mode operating $0.6328 \mu \mathrm{m} \mathrm{He}-\mathrm{Ne}$ gas laser show a tendency to be linearly polarized at right angles to each other $[9,64,65]$, no complete theory has been given yet to describe multi-mode polarization. By treating the two-mode case, we are able to account for mode-mode interaction which is of interest in multi-mode operation.

In Section 6.1 the general expression for the polarization response, derived in Chapter 5, will be used to obtain equations for the modepolarization parameters of a two-mode gas laser. Then, after reconsidering the single-mode behaviour for the various types of laser transitions, we will give in Section 6.2 a theoretical analysis of the mode-polarization behaviour for the case of an isotropic gas laser, oscillating simultaneously in two longitudinal modes. The influence of small cavity anisotropies on the polarization behaviour is discussed in Section 6.3 , both for singlemode and for two-mode operation.

\section{1 derivation of the two-mode equations}

In order to derive equations for the polarization parameters for the two-mode case, we will first write out (5-57) explicitly for this case. Assuming propagation along the $z$-axis, we can substitute $E_{0}=0$ in (5-57). If we indicate the two modes by $n, n^{\prime}=1,2\left(n \neq n^{\prime}\right)$, equation (5-57) reduces to 


$$
\begin{aligned}
& \left(\Omega / \varepsilon_{0}\right) P_{ \pm 1}(n)=-a_{ \pm 1} E_{ \pm 1}(n)+b_{ \pm 1 ; \pm 1, \pm 1, \pm 1}^{n ; n}\left|E_{ \pm 1}(n)\right|^{2} E_{ \pm 1}(n) \\
& +\left(b_{ \pm 1 ; \mp 1, \pm 1, \mp_{1}^{n}}^{n}+b_{ \pm 1 ; \mp_{1}, \mp_{1}, \pm 1}^{n ; n, n, n}\right)\left|E_{\mp 1}(n)\right|^{2} E_{ \pm 1}(n) \\
& +\left(b_{ \pm 1 ; \pm 1, \pm 1, \pm 1}^{n ; n !, n !, n}+b_{ \pm 1 ; \pm 1, \pm 1, \pm 1}^{n ; n !, n, n !}\right)\left|E_{ \pm 1}\left(n^{\prime}\right)\right|^{2} E_{ \pm 1}(n) \\
& +\left(b_{ \pm 1 ; \mp^{n}, \mp 1, \pm 1}^{n ; n^{\prime}, b^{\prime}, n}+b_{ \pm 1 ; \mp^{n}, \pm 1, \eta_{1}^{\prime}}^{n}\right)\left|E_{\mp 1}\left(n^{\prime}\right)\right|^{2} E_{ \pm 1}(n) \\
& +\left(b_{ \pm 1 ; \mp^{n}, \pm 1, \mp_{1}^{\prime}}^{n^{\prime}, n^{\prime}, n} b_{ \pm 1 ; \mp_{1}^{n}, \mp_{1}, \pm 1}^{n^{\prime}, n, n^{\prime}}\right) E_{\mp 1}^{*}\left(n^{\prime}\right) E_{ \pm 1}\left(n^{\prime}\right) E_{\mp 1}(n) .
\end{aligned}
$$

For each of the two modes we introduce the polarization parameters related to the intensity, ellipticity and azimuth:

$$
\begin{aligned}
& I_{n}=\left|E_{-1}(n)\right|^{2}+\left|E_{1}(n)\right|^{2} ; \\
& \sin \left(2 x_{n}\right)=I_{n}^{-1}\left\{\left|E_{-1}(n)\right|^{2}-\left|E_{1}(n)\right|^{2}\right\} ; \\
& u_{n}=\frac{1}{2} \arg \left\{E_{1}(n) E_{-1}^{*}(n)\right\} .
\end{aligned}
$$

The equations of motion for these polarization parameters can be derived, starting from the laser equation (2-26), if we substitute (6-1) and switch to the polarization parameters (6-2). In this derivation we make use of the following symmetry relation, which is valid in the magnetic-fieldfree case:

$$
\begin{aligned}
& n ; n^{\prime}, n^{\prime \prime}, n^{\prime \prime \prime} \\
& b_{Z ; Z,, Z ", Z " \prime}=b_{-Z ;}^{n} ; n^{\prime}, n^{\prime \prime}, n^{\prime \prime},-Z^{\prime \prime},-Z^{\prime \prime}
\end{aligned}
$$

and which can for instance be directly derived from (5-55). It is helpful to introduce the quantities $\left(n, n^{\prime}=1,2\right)$ 


$$
\begin{aligned}
& a_{n}=a_{1}^{n} ; \\
& b_{n n}=\frac{1}{2}\left(\begin{array}{c}
n ; n, n, n \\
1 ; 1,1,1
\end{array}+b_{1 ;-1,1,-1}^{n ; n, n, n}+b_{1 ;-1,-1,1}^{n ; n, n, n}\right) ; \\
& c_{n n}=\frac{1}{2}\left(b_{1 ; 1,1,1}^{n ; n, n, n}+b_{1 ;-1,1,-1}^{n ; n, n, n}-b_{1 ;-1,-1,1}^{n ; n, n, n}\right)
\end{aligned}
$$

and, for $n \neq n$,

$$
\begin{aligned}
& b_{n n^{\prime}}=\frac{1}{2}\left(b^{n ; n^{\prime}, n^{\prime}, n}+b_{1 ; 1,1,1}^{n ; n^{\prime}, n, n^{\prime}}+b_{1 ; 1,1,1}^{n ; n^{\prime}, n^{\prime}, n} \begin{array}{c}
1 ;-1,1,-1 \\
1 ; 1 ; n^{\prime}, n, n^{\prime} \\
1 ;-1,-1,1
\end{array}\right\}(6-4) \\
& \left.+b_{1 ;-1,-1,1}^{n ; n^{\prime}, n^{\prime}, n}+b_{1 ;-1,1,-1}^{n ; n^{\prime}, n, n^{\prime}}\right)
\end{aligned}
$$

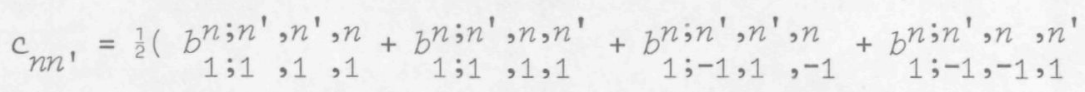

$$
\begin{aligned}
& \left.-b_{1 ;-1,-1,1}^{n ; n^{\prime}, n^{\prime}, n}-b_{1 ;-1,1,-1}^{n ; n^{\prime}, n, n^{\prime}}\right) \\
& d_{n n^{\prime}}=\frac{1}{2}\left(b_{1 ;-1,1,-1}^{n ; n^{\prime}, n^{\prime}, n}+b_{1 ;-1,-1,1}^{n ; n^{\prime}, n, n^{\prime}}\right) .
\end{aligned}
$$

In the case of an isotropic loss tensor, the equations of motion for the polarization parameters of mode $n=1 \mathrm{read}$

$$
\begin{aligned}
2 d_{t} x_{1} & =\frac{1}{2} I_{1} c_{11}^{\prime \prime} \sin 4 x_{1}+I_{2}\left\{\left(c_{12}^{\prime \prime}-d_{12}^{\prime \prime}\right) \cos 2 x_{1} \sin 2 x_{2}\right. \\
& \left.-d_{12}^{\prime} \sin 2\left(u_{1}-u_{2}\right) \cos 2 x_{2}+d_{12}^{\prime \prime} \cos 2\left(u_{1}-u_{2}\right) \sin 2 x_{1} \cos 2 x_{2}\right\} \\
2 \cos 2 x_{1} d_{t} u_{1} & =\frac{1}{2} I_{1} c_{11}^{\prime} \sin 4 x_{1}+I_{2}\left\{\left(c_{12}^{\prime}-a_{12}^{\prime}\right) \cos 2 x_{1} \sin 2 x_{2}\right. \\
& \left.+d_{12}^{\prime \prime} \sin 2\left(u_{1}-u_{2}\right) \cos 2 x_{2}+d_{12}^{\prime} \cos 2\left(u_{1}-u_{2}\right) \sin 2 x_{1} \cos 2 x_{2}\right\}
\end{aligned}
$$




$$
\begin{aligned}
\left(I_{1}^{-1}\right) d_{t} I_{1} & =a_{1}^{\prime \prime}-\Gamma^{\prime}-I_{1}\left(b_{11}^{\prime \prime}-c_{11}^{\prime \prime} \sin ^{2} 2 x_{1}\right)-I_{2}\left\{b_{12}^{\prime \prime}-d_{12}^{\prime \prime}\right. \\
& \left.-\left(c_{12}^{\prime \prime}-d_{12}^{\prime \prime}\right) \sin 2 x_{1} \sin 2 x_{2}+d_{12}^{\prime \prime} \cos 2\left(u_{1}-u_{2}\right) \cos 2 x_{1} \cos 2 x_{2}\right\},
\end{aligned}
$$

while the corresponding equations for mode $n=2$ are obtained after interchanging the mode indices 1 and 2 . In (6-7), $\Gamma^{\prime}$ is the isotropic cavity loss, which is taken equal for both modes. It is noted that in the righthand sides of $(6-5)-(6-7)$ the angles $v_{1}$ and $v_{2}$ show up only via the difference $u_{1}-u_{2}$. This is to be expected in the isotropic case under consideration. It is therefore useful to consider the equation of motion for $v_{1}-u_{2}$, rather than the respective equations for $v_{1}$ and $u_{2}$ themselves. This equation reads

$$
2 \cos 2 x_{1} \cos 2 x_{2} a_{t}\left(u_{1}-u_{2}\right)=g_{12}-g_{21},
$$

where, for $n, n^{\prime}=1,2$ and $n \neq n^{\prime}$,

$$
\begin{aligned}
& g_{n n^{\prime}}=I_{n}\left\{\frac{1}{2}\left(c_{n n}^{\prime}-c_{n^{\prime} n}^{\prime}+d_{n^{\prime} n}^{\prime}\right) \sin 4 x_{n} \cos 2 x_{n^{\prime}}\right. \\
& \left.+d_{n^{\prime} n^{\prime \prime}} \sin 2\left(u_{n}-u_{n^{\prime}}\right) \cos ^{2} 2 x_{n}-d_{n^{\prime} n^{\prime}} \cos 2\left(u_{n}-u_{n^{\prime}}\right) \sin 2 x_{n^{\prime}} \cos ^{2} 2 x_{n}\right\} .
\end{aligned}
$$

Explicit expressions for the $a, b, c$ and $d$ coefficients in (6-4) can be obtained from (5-55), by taking $h=0$, and by using (5-37)-(5-41). The result is

$$
\begin{aligned}
& a_{n}=\left(\varepsilon_{0} \hbar \lambda_{\mathrm{d}}\right)^{-1} \Omega \overline{\mathrm{N}} p^{2} F_{1} J_{0}\left(s_{n}\right) ; \\
& b_{n n}=\frac{\Omega \overline{\mathrm{N}} p^{4}}{16 \varepsilon_{0} \hbar^{3} \lambda_{\mathrm{d}}^{3}} F_{3}\left(1 / \lambda_{\mathrm{a}}+1 / \lambda_{\mathrm{b}}\right) J\left(s_{n}, s_{n}\right) ; \\
& c_{n n}=\left\{\left(F_{3}-F_{2}\right) / F_{3}\right\}_{n n} ;
\end{aligned}
$$


and, for $n \neq n^{\prime}$,

$$
\begin{aligned}
& b_{n n^{\prime}}=\frac{\Omega \overline{\mathrm{N}} p^{4}}{16 \varepsilon_{0} \hbar^{3} \lambda_{d}^{3}} F_{3}\left\{\left(1 / x_{a}+1 / x_{b}\right) J\left(s_{n}, s_{n^{\prime}}\right)\right. \\
& \left.+\left\{\frac{1}{\chi_{a}-i\left(s_{n}-s_{n^{\prime}}\right)}+\frac{}{\chi_{b}-i\left(s_{n}-s_{n^{\prime}}\right)}\right\} K\left(s_{n}, s_{n},\right)\right\} \text {; } \\
& c_{n n^{\prime}}=\frac{\Omega \overline{\mathrm{N}} p^{4}}{16 \varepsilon_{0} \hbar^{3} \lambda_{\mathrm{d}}^{3}}\left\{\left(F_{3}-F_{2}\right)\left(1 / \lambda_{\mathrm{a}}+1 / \lambda_{\mathrm{b}}\right) J\left(s_{n}, s_{n^{\prime}}\right)\right. \\
& \left.+\left\{\frac{F_{2}-F_{4}}{\lambda_{a}-i\left(s_{n}-s_{n^{\prime}}\right)}+\frac{F_{4}}{\lambda_{b}-i\left(s_{n}-s_{n}\right)}\right\} K\left(s_{n}, s_{n},\right)\right\} ; \\
& d_{n n^{\prime}}=\frac{\Omega \bar{N} p^{4}}{32 \varepsilon_{0} \hbar^{3} \lambda_{d} 3}\left\{\frac{F_{3}+F_{4}-F_{2}}{\lambda_{a}}+\frac{F_{3}-F_{4}}{\lambda_{b}}\right\} J\left(s_{n}, s_{n^{\prime}}\right) \\
& \left.+\left\{\frac{F_{3}-F_{4}}{\lambda_{a}-i\left(s_{n}-s_{n}\right)}+\frac{F_{3}+F_{4}-F_{2}}{\lambda_{b}-i\left(s_{n}-s_{n^{\prime}}\right)}\right\} K\left(s_{n}, s_{n^{\prime}}\right)\right\},
\end{aligned}
$$

where $J\left(s, s^{\prime}\right)$ and $K\left(s, s^{\prime}\right)$ are defined by

$$
\begin{aligned}
& J\left(s, s^{\prime}\right)=-i\left\{J_{1}\left(s, s^{\prime}\right)+J_{1}\left(s,-s^{\prime}\right)+J_{2}\left(s, s^{\prime}\right)+J_{2}\left(s,-s^{\prime}\right)\right\} ; \\
& K\left(s, s^{\prime}\right)=-i\left\{J_{1}\left(s,-s^{\prime}\right)+J_{2}(s, s)\right\} .
\end{aligned}
$$

The function $J_{0}(s)$ which describes the dependence of the coefficient $a_{n}$ on detuning and is proportional to the function $K_{0}(\omega-\Omega)$ defined in (3-71). The functions $J_{1}\left(s, s^{\prime}\right)$ and $J_{2}\left(s, s^{\prime}\right)$ have been defined in Section 5.3. The function $J\left(s, s^{\prime}\right)$ satisfies

$$
J\left(s, s^{\prime}\right)=J\left(s,-s^{\prime}\right) ; \quad J^{\prime}\left(s, s^{\prime}\right)=-J^{\prime}\left(-s,-s^{\prime}\right) ; \quad J^{\prime \prime}\left(s, s^{\prime}\right)=J^{\prime \prime}\left(s^{\prime}, s\right) \geq 0 \text {. }
$$




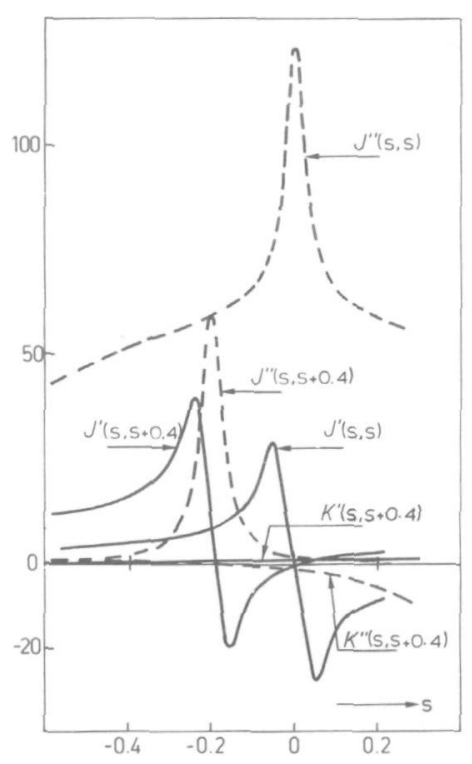

Fig. 6.1 Real (-) and imaginary (----) parts of the functions $J(s, s), J(s, s+0.4)$ and $K(s, s+0.4)$, corresponding to the case of two-mode operation with mode spacing $\left(\omega_{2}-\omega_{1}\right) / \lambda_{d}=$ 0.4 . The transition line width parameter $\lambda=0.0287$

For $s^{\prime} s^{\prime}, J\left(s, s^{\prime}\right)$ is proportional to the function $K(\omega-\Omega)$, defined in (3-72). In Fig. 6.1 the real and imaginary parts of $J(s, s), J\left(s, s^{\prime}\right)$ and $K\left(s, s^{\prime}\right)$ are plotted for a case in which the mode spacing $\left|s-s^{\prime}\right|$ equals 0.4 times the Doppler width and in which $\chi=0.0287$. The resonance peak in $J^{\prime \prime}(s, s)$ at $s=0$ is due to self-saturation of one single mode; the resonance peak in $J^{\prime \prime}(s, s+0.4)$ at $s=-0.2$ is caused by cross-saturation of two modes. According to $(6-13)-(6-15)$ the function $K\left(s, s^{\prime}\right)$ always has to be multiplied by factors like $\left\{\lambda_{\alpha}-i\left(s_{n}-s_{n}\right)\right\}^{-1}$, where $n \neq n^{\prime}$, while the function $J\left(s, s^{\prime}\right)$ is multiplied by factors 1 ike $1 / \lambda_{\alpha}$. So, if we restrict ourselves to cases in which the mode spacing is large with respect to the line-width parameters - which is usually the case- it is consistent with other approximations already made in Chapter 5 to disregard the contributions proportional to $K$ in the right-hand sides of (6-13)-(6-15). If this approximation is applied, equations $(6-11)-(6-15)$ reduce to 


$$
\begin{aligned}
& b_{n n^{\prime}}=-\frac{\bar{\Omega} p^{4}}{16 \varepsilon_{0} \hbar^{3} \lambda_{d}^{3}} F_{3}\left(1 / \lambda_{a}+1 / \lambda_{b}\right) J\left(s_{n^{\prime}}, s_{n^{\prime}}\right) ; \\
& c_{n n^{\prime}}=\left\{\left(F_{3}-F_{2}\right) / F_{3}\right\} b_{n n^{\prime}} ; \\
& d_{n n^{\prime}}=\frac{\Omega \bar{N} p^{4}}{32 \varepsilon_{0} \hbar^{3} \lambda_{d}^{3}}\left\{\frac{F_{3}+F_{4}-F_{2}}{\lambda_{a}}+\frac{F_{3}-F_{4}}{\lambda_{b}}\right\} J\left(s_{n^{\prime}}, s_{n^{\prime}}\right), \quad\left(n \neq n^{\prime}\right),
\end{aligned}
$$

for $n, n^{\prime}=1,2$. Let us recall the F-quantities. According to (5-37)-(5-41) we have

$$
\begin{aligned}
& F_{1}= \begin{cases}(2 / 3)(j+1)(2 j+1)(2 j+3), & j \leftrightarrow j+1 ; \\
(2 / 3) j(j+1)(2 j+1), & j \rightarrow j ; ;\end{cases} \\
& F_{3}= \begin{cases}(2 / 5) F_{1}\left(4 j^{2}+8 j+5\right), & j \leftrightarrow j+1 ; \\
(2 / 5) F_{1}\left(3 j^{2}+3 j-1\right), & j \rightarrow j ;\end{cases} \\
& F_{3}-F_{2}= \begin{cases}-(4 / 5) F_{1} j(j+2), & j \leftrightarrow j+1 ; \\
(2 / 5) F_{1}(j+2)(j-1), & j \rightarrow j ;\end{cases} \\
& (1 / 5) F_{1} j(2 j-1), \quad j_{\mathrm{a}}=j \rightarrow j_{\mathrm{b}}=j+1 \text {; } \\
& F_{3}+F_{4}-F_{2}=\left\{\begin{array}{lr}
(1 / 5) F_{1}(j+2)(2 j+5), & j_{\mathrm{a}}=j+1 \rightarrow j_{\mathrm{b}}=j ; \\
(1 / 5) F_{1}(2 j-1)(2 j+3), & j \rightarrow j ;
\end{array}\right. \\
& E_{3}-E_{4}=\left\{\begin{array}{lc}
(1 / 5) F_{1}(j+2)(2 j+5), & j_{a}=j \rightarrow j_{b}=j+1 \\
(1 / 5) F_{1} j(2 j-1), & j_{a}=j+1 \rightarrow j_{b}=j ; \\
(1 / 5) F_{1}(2 j-1)(2 j+3), & j \rightarrow j .
\end{array}\right.
\end{aligned}
$$


6.2 behaviour of the polarization parameters (isotropic loss tensor)

In order to discuss the polarization behaviour of two modes it is useful to recall the single-mode case. The single-mode ellipticity can be deduced from $(6-5)$ by substituting $I_{2}=0$, which leads to

$$
\mathrm{d}_{t} x_{1}=\frac{1}{4} I_{1} c_{11}^{\prime \prime} \sin 4 x_{1}
$$

This equation was also derived by Van Haeringen [39]. It is easily seen that, in the case of laser operation, i.e., $I_{1}>0$, the stable timeindependent solutions of $(6-26)$ are determined by $F_{3}-F_{2}[$ see $(6-19)]$, namely, $x_{1}=0$ if $F_{3}-F_{2}<0, x_{1}= \pm \pi / 4$ if $F_{3}-F_{2}>0$ and $x_{1}$ is undetermined if $F_{3}-F_{2}=0$. Hence, it follows from (6-23) that a linear state of polarization is predicted for the $\frac{1}{2} \rightarrow \frac{1}{2}$ and the $j \leftrightarrow j+1$ class of transitions $(j>0)$; bistable circular polarization for $j \rightarrow j(j>1)$, and neutral behaviour of $x_{1}$ for $0 \leftrightarrow 1$ and $1 \rightarrow 1$, respectively.

Except for the $0 \leftrightarrow 1$ and $1 \rightarrow 1$ transitions, the predicted polarization preferences are in agreement with experiments [24,26]. It should be emphasized that the above-derived preferences are entirely due to the addition of all third-order quantum-mechanical transition probabilities between the two multiplets involved. In those cases in which this addition leads to neutral behaviour, i.e., the $0 \leftrightarrow 1$ and $1 \rightarrow 1$ transitions, it is essential to follow the refined approach of Chapter 3, where the treatment includes the occurrence of different relaxation rates within one multiplet.

Let us now return to the two-mode case. We have used three different methods to derive the stable time-independent solutions of the set of equations for $x_{n}, u_{n}$ and $I_{n}(n=1,2)$. Two methods are based on the observation that direct inspection of $(6-5)$ and $(6-8)$, in which both $I_{1}$ and $I_{2}$ are assumed to be nonzero and positive, leads to six different combinations $x_{1}, x_{2}$ and $v_{1}-u_{2}$, each of which might correspond to a time-independent solution of the equations. These combinations are 
(1) $x_{1}=x_{2}=0$ and $u_{1}-u_{2}=0$;

(2) $x_{1}=x_{2}=0$ and $v_{1}-v_{2}= \pm \pi / 2$;

(3) $x_{1}=x_{2}=0$ and $v_{1}-v_{2}$ is indifferent;

(4) $x_{1}=x_{2}= \pm \pi / 4$;

(5) $x_{1}=-x_{2}= \pm \pi / 4$;

(6) $x_{1}=-x_{2}$ and $u_{1}-u_{2}= \pm \pi / 2$.

In the first (approximative) method, the stability analysis has been carried out by reduction of the set of differential equations to only one or two relevant differential equations, allowing for an analytic solution of the stability problem. In the second (numerical) method, the entire system of coupled differential equations (including the equations for $I_{1}$ and $I_{2}$ ) is considered. The equations are linearized around the time-independent solutions under consideration and the stability problem is treated by standard methods [66], in which the eigenvalues of the coefficient matrix of the linearized equations play a role. In addition to these two methods, a computer program has been used to integrate the entire system of coupled equations for various starting conditions. All methods appeared to be in full agreement with each other. The results are summarized in Table 6.1 and will be discussed below.

The $j \leftrightarrow j+1 \quad(j>0)$ class of transitions. Each of the two modes is linearly polarized, as would also be the case in the absence of mode-mode interaction. Hence, the only effect due to the interaction between the two modes is on the relative orientation $v_{1}-v_{2}$ of their polarization planes. This is governed by the positive coefficients $d_{21}^{\prime \prime}$ and $d_{12}^{\prime \prime}$ in the right-hand side of (6-8), and it leads to a preference for mutually orthogonal linearly polarized states.

The $\frac{1}{2} \rightarrow \frac{1}{2}$ type transitions. For this class $d_{n n^{\prime}}=0$ which leads to indifference of $u_{1}-u_{2}$ when $x_{1}=x_{2}=0[$ see $(6-8)$ and $(6-9)]$. It can be argued that taking into account a more general relaxation mechanism than that considered in Chapter 5 will lead to very small but positive values of $d_{21}^{\prime \prime}$ and $d_{12}^{\prime \prime}$. This will then result in a very weak preference for orthogonal linearly polarized states. 
Table 6.1 Polarization preferences in an isotropic two-mode gas laser POLARIZATION TYPE OF PREFERENCE TRANSITION

REMARKS

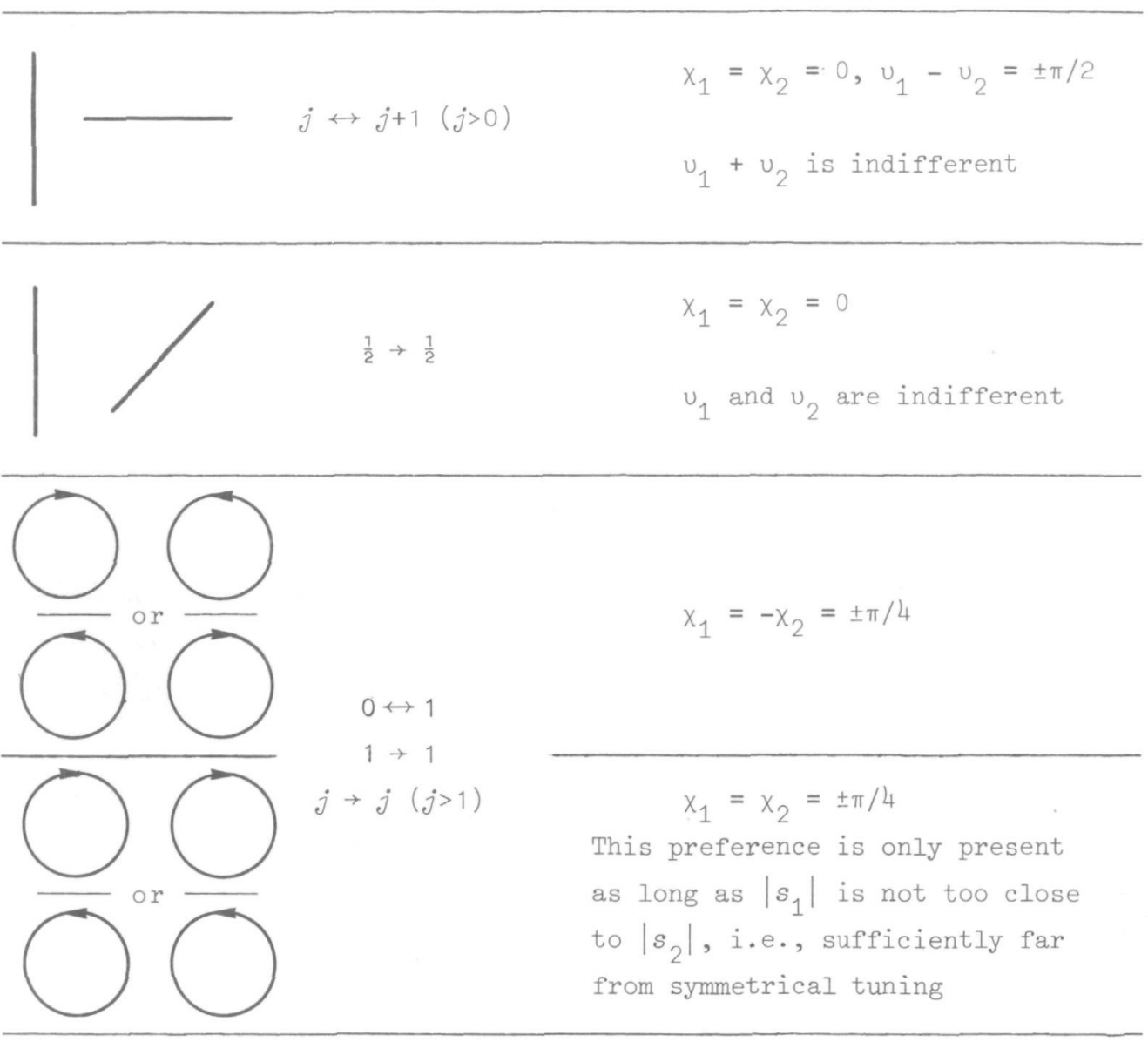

The $j \rightarrow j(j>1)$ class of transitions. Again, the polarization preference of each separate mode is equal to the single-mode preference. The modemode interaction is mainly governed now by the negative coefficients $c_{12}^{\prime \prime}-d_{12}^{\prime \prime}$ and $c_{21}^{\prime \prime}-d_{21}^{\prime \prime}$, respectively, [see (6-5)] and results in a tendency towards oppositely circularly polarized modes. However, it turns out that this tendency is not always effective in preventing circular polarization of the two modes with the same sense of rotation. In particular, if the resonator tuning is sufficiently far from symmetrical tuning, then one 
has in $(6-5):\left(d_{12}^{\prime \prime}-c_{12}^{\prime \prime}\right) I_{2}<<c_{11}^{\prime \prime} I_{1}$, and similarly, $\left(d_{21}^{\prime \prime}-c_{21}^{\prime \prime}\right) I_{1}<<c_{22}^{\prime \prime} I_{2}$, and this results in a situation in which both $x_{1}=x_{2}= \pm \pi / 4$ and $x_{1}=-x_{2}= \pm \pi / 4$ are stable states. Only in the case of almost symmetrical tuning, i.e., $s_{1} \simeq-s_{2}$, is the mode-mode interaction strong enough to push the system away from $x_{1}=x_{2}= \pm \pi / 4$, such that $x_{1}=-x_{2}= \pm \pi / 4$ is the only stable situation. An exception of the kind described above cannot occur in the $j \leftrightarrow j+1 \quad(j>0)$ case: Due to the degeneracy with respect to the orientation of the polarization plane in the single-mode case, the mode-mode interaction is always effective in fixing the relative orientation of the polarization planes in one and the same way, no matter how weak the interaction is.

The $0 \leftrightarrow 1$ and $1 \rightarrow 1$ transitions. For these transitions one has $d_{n n}^{\prime \prime}>0$. If no interstate relaxation is considered one has also $c_{n n^{\prime}}=0$. If this is the case, mode-mode interaction would then lead to modes with opposite ellipticity (it does not matter which) such that the long axes of the corresponding polarization ellipses are perpendicular. If, however, interstate relaxations are taken into account, as should be done (see Chapter 3), this will result in a nonzero, positive $c_{n n}^{\prime \prime}(n=1,2)$, which will bring these transitions into the $j \rightarrow j(j>1)$ class. Hence, it follows that for the $0 \leftrightarrow 1$ and $1 \rightarrow 1$ transitions, interstate relaxations result in the same polarization behaviour as the $j \leftrightarrow j(j>1)$ class.

Under very special conditions, namely exactly symmetrical tuning, i.e., $s_{1}=-s_{2}$, and, simultaneously, exactly equal mode intensities, i.e., $I_{1}=I_{2}$, a peculiar result is obtained. In that case each couple of mutually orthogonal elliptical modes happens to be a time-independent solution of the equations of motion, except in the $\frac{1}{2} \rightarrow \frac{1}{2}$ case, in which case the modes have opposite ellipticity while the angle between their long axes is indifferent.

In Figures 6.2 and 6.3 some results of numerical integrations are given for a $1 \rightarrow 2$ and a $2 \rightarrow 2$ transition, respectively. Time evolutions are given for the polarization ellipses of the two coupled modes for some starting conditions. For the $2+2$ transition this demonstrates that both $x_{1}=x_{2}= \pm \pi / 4$ and $x_{1}=-x_{2}= \pm \pi / 4$ are stable, while the starting condition determines which one will be chosen. The parameter $n_{0}$ is a quantity related 

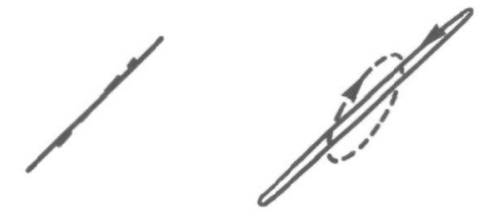

$t=0$

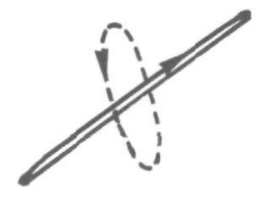

$t=500$ $t=360$

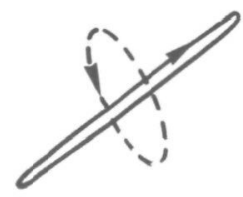

$t=550$

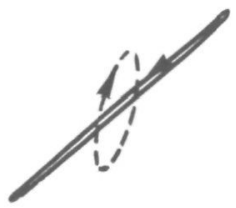

$t=410$

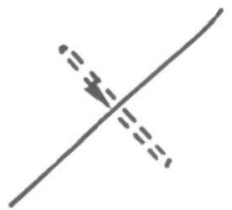

$t=750$

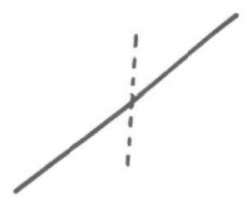

$t=440$

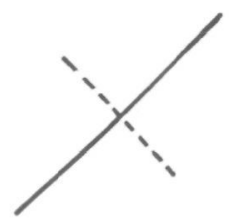

$t=1100$

Fig. 6.2 Time evolution of the two mode-polarization ellipses for a $j_{\mathrm{a}}=1 \rightarrow j_{\mathrm{b}}=2$ transition. The state at $t=0$ is unstable; the final state is stable. The parameters are $s_{1}=-0.1$ and $s_{2}=0.3$ (the first mode has the largest intensity). The unit of time is the cavity life time $1 / \Gamma^{\prime}$, which is chosen equally for the two modes. Other parameters are $\eta_{0}=1.2 ; \lambda_{a}=0.02$; $\lambda_{b}=0.04, \quad x=0.1$

(a)
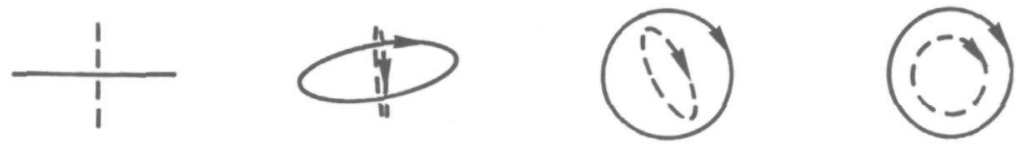

$t=0$

$t=60$

$t=210$

$t=600$

(b)
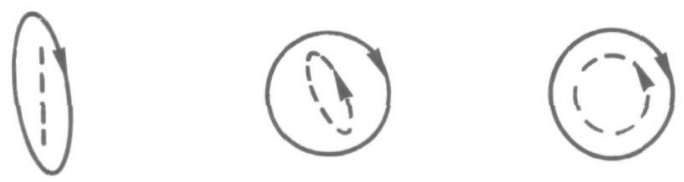

Fig. 6.3 Time evolution of the two mode-polarization ellipses for a $j_{\mathrm{a}}=2 \rightarrow j_{\mathrm{b}}=2$ transition for two different starting conditions. The parameters are $s_{1}=-0.36 ; s_{2}=0.04 ; \eta_{0}=1.2 ; \lambda_{a}=0.0178$; $\lambda_{b}=0.0396 ; \lambda=0.0287$. Time unit as in Fig. 6.2 
to the pump strength. It is defined as

$$
n_{0}=a_{1}^{\prime \prime}\left(s_{1}=0\right) / \Gamma^{\prime}=a_{2}^{\prime \prime}\left(s_{2}=0\right) / \Gamma^{\prime} .
$$

The calculated intensity spectrum of a two-mode, $j_{a}=1 \rightarrow j_{b}=2$ laser is given in Fig. 6.4, where the intensity of one single mode has been drawn as a function of its position within the Doppler-broadened gain profile. The corresponding spectrum of the second mode has not been drawn, as it is essentially equal. The central dip in the intensity curve is the Lamb dip, which is due to interaction between the two travelling waves of the mode under consideration. The other two dips, occurring for symmetrical tuning, are due to mode-mode interaction, that is, each of the travelling waves of the mode considered interacts with the opposite travelling wave of the other mode. Note therefore that the mutual orthogonality is accompanied by a cross-saturation effect in the intensities. The calculation of the intensity spectrum for a $2 \rightarrow 2$ laser yielded a result similar to that in Fig. 6.4 .

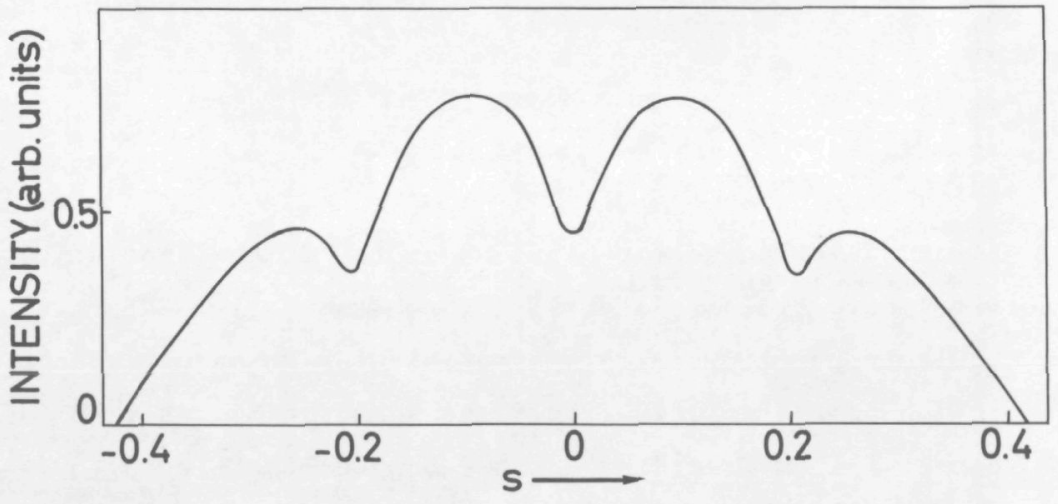

Fig. 6.4 Mode intensity spectrum of a two-mode laser. The intensity (in arbitrary units) of one single mode is plotted as a function of its detuning $s=(\omega-\Omega) / \lambda_{d}$, where $\omega$ is the frequency of the mode. The type of transition is $j_{\mathrm{a}}=1 \rightarrow j_{\mathrm{b}}=2$. The parameters are $\lambda=0.0287 ; \lambda_{a}=0.0178 ; \lambda_{b}=0.0396 ; n_{0}=1.2$; and the mode spacing is $0.4 \lambda_{d}$ 
Let us summarize the results of this section. The various timeindependent solutions of the two-mode equations have been tested with respect to stability. The polarization preferences in an isotropic twomode laser have been obtained and discussed in relation to the singlemode case. As a rule, we have found that cross-saturation effects of two different longitudinal modes induce a preference for orthogonal eigenstates of polarization without, however, changing the single-mode polarization preference of each individual mode. For the $j \leftrightarrow j+1(j>0)$ type of transitions this leads to orthogonal, linear polarizations, regardless of the strength of the mode-mode interaction, which confirms the experimentally wel1-known, mutually orthogonal, linear polarization of a $0.6328 \mu \mathrm{m} \mathrm{He-Ne}$ multi-mode gas laser $(1 \rightarrow 2$ transition).

For the $j \rightarrow j(j>1)$ type of transitions we have found that under special tuning conditions the preference for orthogonality induced by mode-mode interaction is not always effective in preventing the two circularly polarized modes from having the same sense of rotation. At present, there seem to be no experimental results available on the polarization behaviour of multi-mode gas lasers operating on transitions other than $j \leftrightarrow j+1 \quad(j>0)$.

\section{3 the influence of a weak cavity anisotropy}

A weak cavity anisotropy influences the state of polarization of the electromagnetic field in a gas laser substantially. For instance, a linear phase anisotropy (1.p.a.) is responsible for an abrupt change in the orientation of the polarization plane when tuning a single-mode He-Ne 0.6328 or $1.152 \mu \mathrm{m}$ laser through the Doppler profile [37,39,44]. In this section we will restrict ourselves to the interesting practical cases of $j \leftrightarrow j+1 \quad(j>0)$ types of transitions, which include the above-mentioned Ne transitions. First we will reconsider the single-mode polarization behaviour in the presence of an 1.p.a. Then, with this in mind, it is easy to discuss the effect of an 1.p.a. in the two-mode case. A similar discussion can be given concerning the effect of a linear amplitude anisotropy (1.a.a.). 
The single-mode equations for the ellipticity and the azimuth in the presence of an 1.p.a. of magnitude $\Gamma_{p}$ can be deduced from $(6-5)-(6-7)$ by first taking $I_{2}=0$, and, second, by extending the right-hand sides with the resonator-induced contributions, given by $(B-6)-(B-8)$ with $\Gamma_{a}=\Gamma_{c}=0$. If we omit the mode index, the equations read

$$
\begin{aligned}
& 2 d_{t} x=-\Gamma_{p} \sin 2\left(u-\theta_{p}\right)+\frac{1}{2} I c " \sin 4 x ; \\
& 2 \cos 2 x d_{t} u=\Gamma_{p} \cos 2\left(u-\theta_{p}\right) \sin 2 x+\frac{1}{2} I c^{\prime} \sin 4 x ; \\
& I^{-1} d_{t} I=a^{\prime \prime}-\Gamma^{\prime}-I\left(b^{\prime \prime}-c^{\prime \prime} \sin ^{2} 2 x\right) .
\end{aligned}
$$

Stable, time-independent solutions of (6-28)-(6-29) are

(1) $x=0, U=\theta_{p}$ if $c^{\prime \prime}<0, \Gamma_{p}+I C^{\prime}>0$;

(2) $x=0, u=\theta_{p} \pm \pi / 2$ if $c^{\prime \prime}<0, \Gamma_{p}-I c^{\prime}>0$,

while in both cases the intensity is given by

$$
I=\left(a^{\prime \prime}-\Gamma^{\prime}\right) / b^{\prime \prime} \text {. }
$$

For the $j \leftrightarrow j+1 \quad(j>0)$ transitions considered here, the condition $c^{\prime \prime}<0$ holds $[$ see $(6-19,23)]$. The remaining conditions $\Gamma_{p} \pm I C^{\prime}>0$ can best be discussed by regarding some plots of the quantity $I C^{\prime}$ as a function of detuning s. In Fig. 6.5 the dimensionless quantity $I C^{\prime} / \Gamma^{\prime}$ is given as a function of $s$, for three different values of the excitation density $\eta_{0}=a^{\prime \prime}(s=0) / \Gamma^{\prime}$. The curves are antisymmetric in $s$. Of special importance are the intersections of the curves with the horizontal lines corresponding to $\pm \Gamma_{p} / \Gamma^{\prime}$, i.e., the values of $s$ for which $I C^{\prime}= \pm \Gamma_{p}$. If there are no such intersections, that is, if $\left|I_{C^{\prime}}\right|<\Gamma_{p}$ for all $\mathrm{s}$, then both solutions, (1) and (2), are stable for all $\mathrm{s}$. This implies that the laser mode is bistable with respect to the orientation of its polarization plane. The possible occurrence of this bistability depends, apart from the excitation density $n_{0}$, on the strength $\Gamma_{\mathrm{p}}$ of the $1 . \mathrm{p} \cdot \mathrm{a}$. 


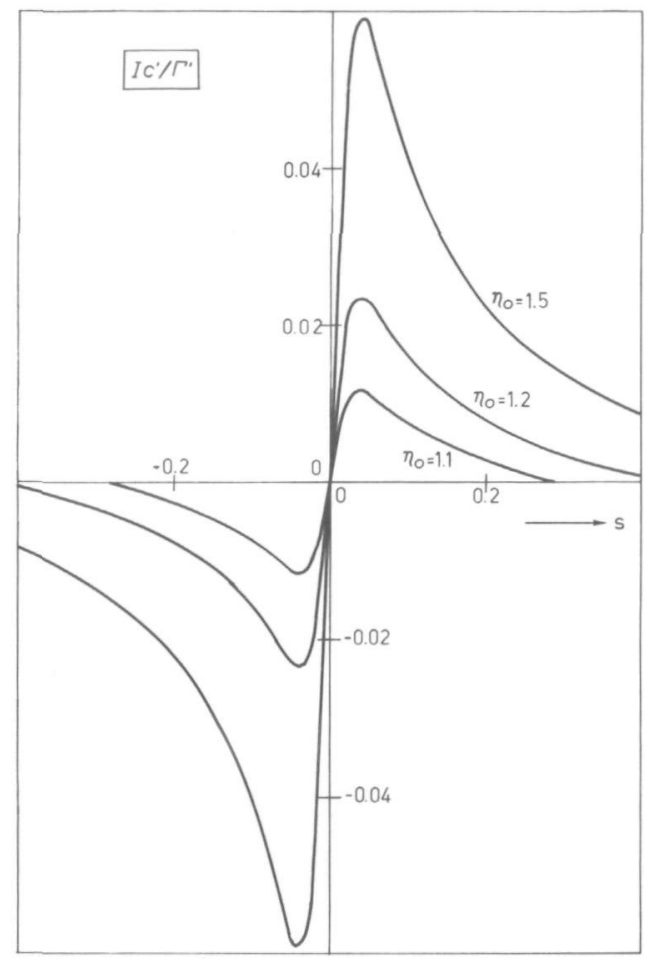

Fig. 6.5 The dimensionless quantity $I C^{\prime} / \Gamma^{\prime}$ as a function of $s$ for three different values of the excitation density $n_{0}$. The parameter values are $\lambda_{=0.0287 ;} \lambda_{\mathrm{a}}=0.0178 ; \lambda_{\mathrm{b}}=0.0396$; $j_{\mathrm{a}}=1 ; j_{\mathrm{b}}=2$

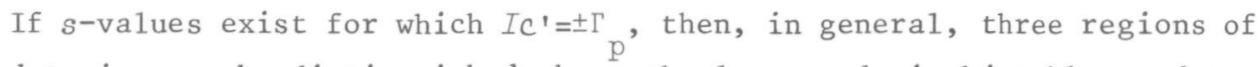
detuning can be distinguished where the laser mode is bistable, and two regions where only one of the two solutions (1) and (2) is stable. This is schematically indicated in Fig.6.6. It follows from this figure that, when tuning a laser mode through the Doppler profile from left to right, an abrupt change of $u$ over $\pi / 2$ occurs at $s_{a}$, whereas tuning in the other direction leads to a polarization flip at $-s_{2}$. The bistability region around $s=0$ and the corresponding polarization flips have indeed been observed [33]. To our knowledge, no experimental reports concerning the two other bistability regions exist. 


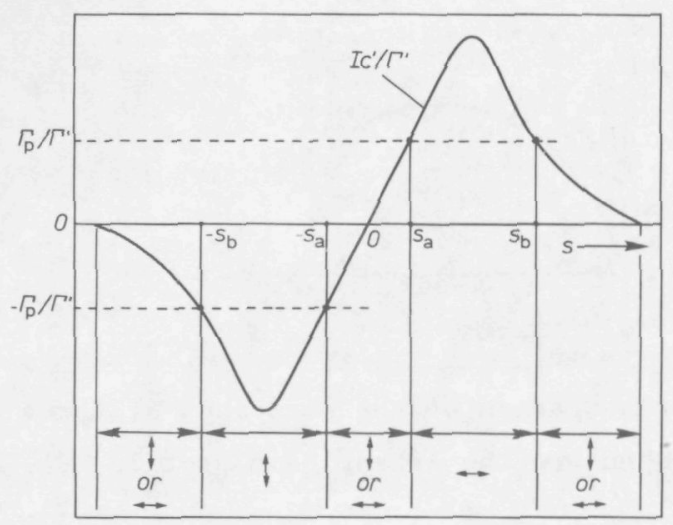

Fig.6.6 Schematic illustration of the respective stability regions in a single-mode gas laser with a linear phase anisotropy of magnitude $\Gamma_{p}$. Here $\downarrow$ means that $v=\Theta_{p}$, while $\leftrightarrow$ means that $u=\theta_{p} \pm \pi / 2$

Let us now study the effect of an 1.p.a. in a two-mode laser, assuming for simplicity that the two modes have exactly the same loss tensor. In Fig.6.7 the (1inear) gain function $a^{\prime \prime}(s)-\Gamma^{\prime}$ is schematically sketched in the $s$-interval for which this function assumes positive values (i.e., the duty cycle). In this figure, the positions of three equidistant modes are also indicated for a case in which modes 2 and 3 have frequencies within the duty cycle, whereas mode 1 lies outside this region. In this case, only modes 2 and 3 will be excited. Tuning of the laser will correspond to shifting of the three modes with respect to the gain profile, while their mutual distance $\Delta s$ remains unaltered, at least in a good approximation. In order to exclude the possibility of simultaneous excitation of more than two modes we will assume that the duty cycle is smaller than $2 \Delta s$. Let us first neglect the effect of mode interaction; the preceding single-mode analysis can then be applied to each mode separately. We have to distinguish between the two possible cases in which either (a) $\Delta s<s_{\mathrm{a}}+s_{\mathrm{b}}$ or (b) $\Delta s>s_{a}+s_{b}$, which leads to the respective diagrams (a) and (b) of Fig.6.8. In these diagrams the orientations of two planes of polarization are indicated as a function of the detuning parameter $s_{2}$ of mode 2 . We recall that in Fig.6.8 the effect of mode-mode interaction has been omitted and the question now arises of what this effect will be. 


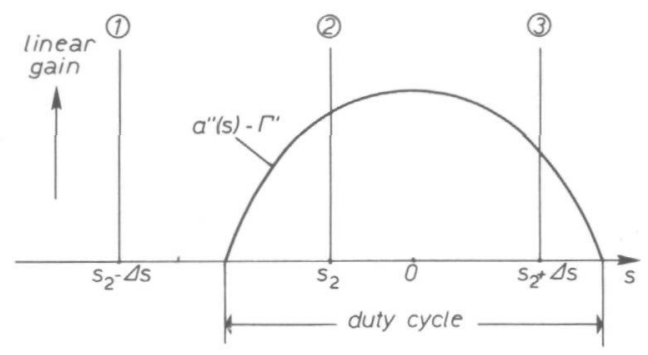

Fig.6.7 Schematic representation of the positions of three equidistant modes with respect to the linear gain profile

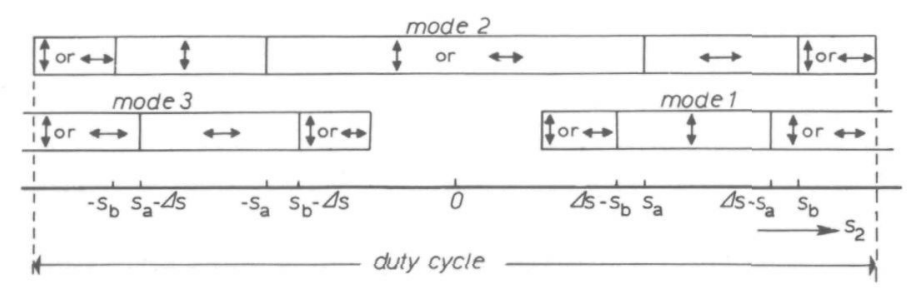

$$
\frac{\Delta s<s_{a}+s_{b}}{(a)}
$$

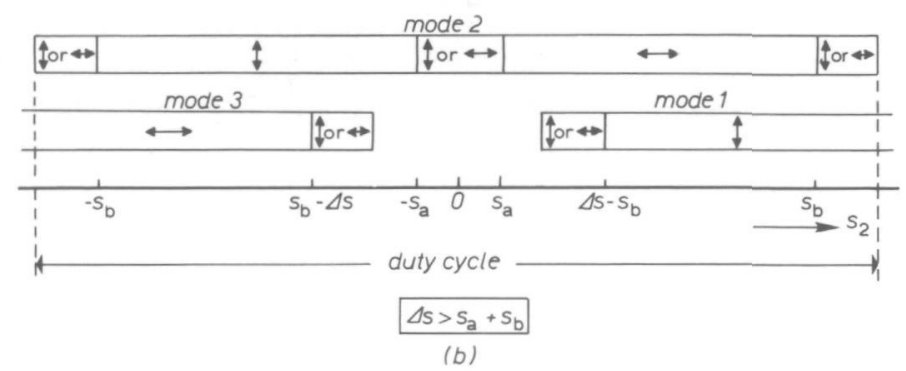

Fig.6.8 Diagrammatic representation of the stable orientations of the planes of polarization of the three different modes in Fig.6.7 as a function of the detuning parameter $s_{2}$ of mode 2 . Here, the effects due to mode-mode interaction have not yet been taken into account. 
From Fig.6.8 it can be deduced that it is always possible to combine the two polarization planes such that they are mutually orthogonal. It is therefore easy to predict what will happen if, in the case of diagram (a), the laser mode 2 is tuned from left to right through the duty cycle. This is indicated in Fig.6.9. Let, for instance, mode 3 be initially present with mode polarization plane $v_{3}=\theta_{p}$ (one of the two possibilities). It is then seen that maintenance of mutual orthogonality requires a combined polarization flip of modes 2 and 3 at $s_{2}=-s_{b}$, and a similar flip of modes 1 and 2 at $s_{2}=\Delta s-s_{b}$. The other cases can also be analyzed in this way. In the case (b) of Fig.6.8 the possible flips will occur in the singlemode region, i.e., at $s_{2}=s_{a}$ (tuning from left to right) and $s_{2}=-s_{a}$ (tuning from right to left).

In order for these polarization flips to really occur, the magnitude $\Gamma_{p}$ of the 1.p.a. should not be too large. That is to say, if $\Gamma_{p}$ is too large, no detuning values $s_{\mathrm{a}}$ and $s_{\mathrm{b}}$ exist (this corresponds to the overall bistability in the single-mode case). In that case it may happen that each of the solutions $v_{1}=v_{2}=\theta_{p} ; v_{1}=v_{2}=\theta_{p} \pm \pi / 2 ; v_{1}=v_{2} \pm \pi / 2=\theta_{p}$ and $v_{1} \pm \pi / 2=$ $\mathrm{U}_{2}=\Theta_{\mathrm{p}}$ is stable for all detuning values.

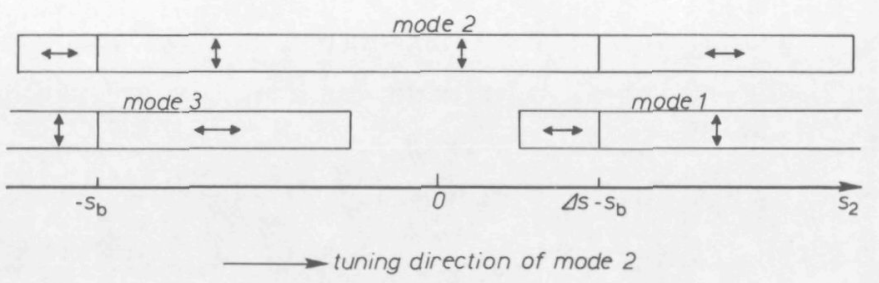

Fig.6.9 Diagrammatic representation of the orientations of the mode polarization planes in a two-mode laser when tuning from left to right. The effect of mode interaction is included. Two combined polarization flips will occur, namely for $s_{2}=-s_{\mathrm{b}}$ and for $s_{2}=\Delta s-s_{\mathrm{b}}$, if the initial polarization direction of mode 3 is as indicated 


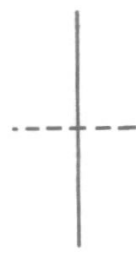

$t=0$

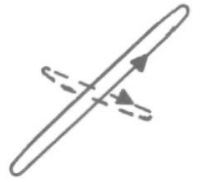

$t=2000$

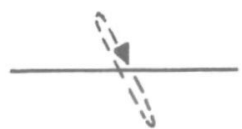

$t=4000$

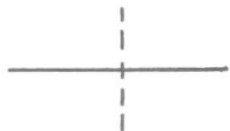

$t=7000$

Fig.6.10 Mode-polarization ellipses at four different times $t$ ( in units $\Gamma^{-1}$ ) during a polarization flip in a two-mode gas laser. The excitation density $n_{0}=1.3$. The mode corresponding to the solid lines has detuning parameter $s_{2}=-0.05$; the other one corresponding to the broken lines has $s_{1}=-0.45$. The 1.p.a. has magnitude $\Gamma_{p}=0.01 \Gamma^{\prime}$. The line width parameters are $\lambda_{0}=0.0287 ; \lambda_{a}=0.0178 ; \lambda_{b}=0.0396$.

A numerical stability analysis applied to the complete set of equations has confirmed the above-stated conclusions. The result of an analysis of the time development of the mode-polarization ellipses during a polarization flip is visualized in Fig.6.10. This result has been obtained by numerical integration of the complete set of equations.

We conclude this chapter by giving some short remarks concerning the effects of a linear amplitude anisotropy (1.a.a.), assumed to be equal for both modes. In the single-mode laser the mode will be 1 inearly polarized along the low-loss main axis,i.e., $v=\Theta_{a}$. This preference is independent of detuning, contrary to the 1.p.a. case. In the two-mode case the mode with the largest intensity will take position along the low-loss main axis, while if the magnitude $\Gamma_{a}$ of the 1.a.a. is not too large, the less intense mode will stand along the high-loss main axis. It follows that in this case also polarization flips may occur when the laser is tuned through the gain profile. If, however, $\Gamma_{a}$ is relatively large compared to the mode-mode interaction strength, both modes will tend to choose their orientations along the low-loss main axis. An extreme example of such a case is the Brewster-window laser. The above qualitative 
considerations have been confirmed by means of a numerical stability analysis.

In common gas lasers, not provided with Brewster windows or other strong1y polarizing components, an 1.p.a. is the main anisotropy with relative magnitude of the order of at most $5 \%$ of the isotropic loss $\Gamma^{\prime}$, while the nagnitude of the $1 . a . a$. is still an order smaller $(0.1-1 \%$ of $\left.\Gamma^{\prime}\right)[39,49]$. 


\section{MODE POLARIZATION IN TRANSVERSE MAGNETIC FIELDS (SINGLE- MODE CASE)}

Several experiments have been performed on single-mode gas lasers subject to either axially or transversally applied magnetic fields (see, for instance, References $[8,12,13,17,22,30,42,43,48,49])$. Among these, especially the experiments in an axial field have been extensively theoretically discussed $[31,38-41]$ which has 1 ed to a satisfactory description of these experiments. The situation is different in the case of transversally applied magnetic fields. Here, the case of a $j=1 \rightarrow j=0$ type of laser transition has been theoretically discussed by Durand [30] and by Culshaw \& Kannelaud [31]. Almost no attention has been devoted to other types of transitions, except in the papers by Sargent et al. [40,41]. However, when confronting this theory with a set of experiments, explicitly discussed further on, it is in one case impossible to obtain satisfactory agreement (as it will turn out to be necessary to consider the presence of magnetic-field-free regions of active medium); in the other cases it is by no means clear how to apply the general theory in an appropriate way.

In view of the poor status of the existing theory, we will give in the present chapter a rather extensive treatment of polarization phenomena in single-mode gaslasers, subject to a transverse magnetic field. The effects of small cavity anisotropies will be discussed as well. In Section 7.1 the case of a transverse magnetic field applied over the entire active medium is considered. By taking into account the phase-locking effect at weak magnetic fields (Zeeman-splitting frequency smaller than natural line widths of atomic levels), our treatment corrects the result of Reference [41].

The configuration considered in Section 7.2 involves a transverse magnetic field applied to a fraction of the active medium, the other part being magnetic-field free. It will be shown that, if the magnetic-field affected region of medium is not too large, the field-free region of medium causes phase locking for all values of the magnetic-field magnitude and detuning. 
In the last two sections the additional influence of small resonator anisotropies is discussed. In Section 7.3 the apparent discrepancies between the experiments of Dumont \& Durand [13] and of Settles \& Heer [43] on the one hand, and the existing theory on the other hand, will be shown to disappear when the presence of resonator anisotropies of reasonable magnitudes is assumed. In Section 7.4 we will discuss the sizeable influence that small resonator anisotropies can have on the output of a single-mode gas laser, which is subject to large magnetic fields.

7.1 transverse magnetic field applied to the entire resonator (isotropic Zoss tensor)

The expressions for the time evolution of the polarization parameters $R, \theta$, I are obtained by considering the combined effect of the mediuminduced contributions (2-60)-(2-62) and the resonator-induced contributions (B-9)-(B-11) (taking $\left.\Gamma_{a}=\Gamma_{p}=\Gamma_{c}=0\right)$. The equations of motion read

$$
\begin{aligned}
2 \mathrm{~d}_{t} R=\sin 2 R\left\{-\alpha_{1}^{\prime \prime} / 2+I\right. & -\gamma^{\prime \prime} \cos 2 \Theta \cos 2 R+\gamma^{\prime} \sin 2 \Theta-\gamma_{1}^{\prime} \sin 2 \Theta \cos 2 R \\
& \left.\left.+\gamma_{1}^{\prime \prime} \cos 2 \theta+\beta_{2}^{\prime \prime}+\delta^{\prime \prime} \cos 2 R\right)\right\} ;
\end{aligned}
$$

$\sin 2 R d_{t} \Theta=\sin 2 R\left\{\alpha_{1}^{\prime} / 2+I\left(\gamma^{\prime} \cos 2 \theta \cos 2 R+\gamma^{\prime \prime} \sin 2 \theta-\gamma_{1}^{\prime \prime} \sin 2 \theta \cos 2 R\right.\right.$

$$
\left.\left.-\gamma_{1}^{\prime} \cos 2 \theta-\beta_{2}^{\prime}-\delta^{\prime} \cos 2 R\right)\right\} \text {; }
$$

$$
\begin{aligned}
(1 / I) d_{t} I=\alpha^{\prime \prime}-\Gamma^{\prime}-\alpha_{1}^{\prime \prime} & \sin ^{2} R-I\left\{\beta^{\prime \prime}-\beta_{1}^{\prime \prime} \sin ^{2} R\right. \\
& \left.+\left(\gamma^{\prime \prime} \cos 2 \theta+\gamma_{1}^{\prime} \sin 2 \theta-\delta^{\prime \prime}\right) \sin ^{2} R \cos ^{2} R\right\},
\end{aligned}
$$

the only remaining resonator contribution being the isotropic loss term $\Gamma^{\prime}$ in (7-3). Expressions for the $\alpha, \beta, \gamma$ and $\delta$-coefficients are given in Appendix C. Among all coefficients, only $\alpha$ and $\beta$ are independent of the magnetic field. The other coefficients are symmetric functions of the magnetic-field parameter $h=\mathrm{H} / \lambda_{\mathrm{d}}$. The real parts are antisymmetric functions of the detuning parameter $s$; the imaginary parts are symmetric in $s$. 
The indexed coefficients, i.e., $\alpha_{1}, \beta_{1}, \beta_{2}$ and $\gamma_{1}$, vanish when $h=0$, while $\gamma$ and $\delta$ then coincide. The coefficients $\gamma$ and $\gamma_{1}$ are only relevant as long as the Zeeman-splitting frequency does not exceed the natural line widths, i.e., when $|h|<\lambda_{a}, \lambda_{b}$; at larger magnetic-field magnitudes they go rapidly to zero. Plots of the various coefficients as a function of $s$ are given in Fig.7.1. The plots refer to the $s=0$ case, in which the real parts of the coefficients all vanish.

For small magnetic-field magnitudes, i.e., if $|h| \leqslant \lambda_{a}, \lambda_{b}$, equations (7-1) -(7-3) admit stable, time-independent solutions for $I, R$ and $\Theta$. In this $h$-region, the right-hand side of (7-2) is dominated by the term $I \gamma^{\prime \prime} \sin 2 \theta$, which leads to a final state in which the phase angle $\theta$ is locked at a constant value. In general there are even two such solutions, since if a stable solution for $\theta$ exists, then $\Theta \pm \pi$ is a stable solution too, with the same values for $I$ and $R$.

At moderate and large magnetic fields, that is, $|h| \gg \lambda_{a}, \lambda_{b}$, the coefficients $\gamma$ and $\gamma_{1}$ in $(7-1)-(7-3)$ are very small and can be ignored. Hence, in that case the equations for $d_{t} I$ and $d_{t} R$ are no longer coupled to the $d_{t} \theta$ equation and, after taking the $\gamma^{\prime} s$ equal to zero, (7-2) is seen to describe a time-dependent phase difference, at least for nonzero

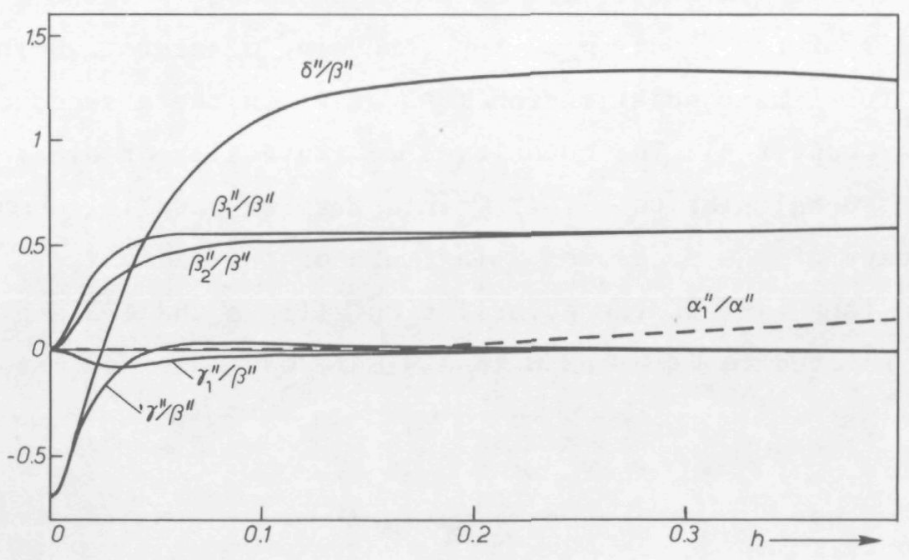

Fig.7.1 The imaginary parts of the various $h$-dependent coefficients as a function of $h$. The parameters are $s=0 ; j_{\mathrm{a}}=1 ; j_{\mathrm{b}}=2$; $\lambda=0.0287 ; \lambda_{a}=0.0178 ; \lambda_{b}=0.0396$ 
detuning values. However, (7-1) and (7-3) are found to have timeindependent, stable solutions for $I$ and $R$. Once this stationary state is reached, the phase angle $\Theta$ is uniformly changing in time.

In a relatively small magnetic-field region for which the Zeeman splitting is approximately equal to the natural line widths, that is, in between the two above-mentioned field regions, $\gamma$ and $\gamma_{1}$ are small but not negligible. In this region $\left|\gamma^{\prime \prime}\right|$ is not large enough anymore to lock $\theta$ in (7-2) to a constant value, which results in a complicated timedependent behaviour of $I, R$ and $\theta$, at least for detuning values not too close to zero. Approximate solutions are easily obtained for this state, by ignoring the $\gamma$-terms in $(7-1)-(7-3)$, or, with the same result, by taking the mean value of the sine and cosine of twice the phase angle over a period. In this way, (7-1) and (7-3) are no longer coupled to (7-2), and an estimate can be obtained for the final-state values of $I$ and $R$. Then, (7-2) can be integrated analytically, as described in Appendix D, which leads to an approximated, time-independent value of the time derivative $W$ of $\theta$.

The results of the analysis of (7-1)-(7-3) are collected in Fig.7.2. Plots of a number of quantities are given, which characterize the state of polarization of the laser mode as a function of the magnetic-field magnitude, for two values of the detuning parameter $s=0$ and $s=0.15$. The transition is of the $j_{\mathrm{a}}=1 \rightarrow j_{\mathrm{b}}=2$ type, showing a saturation-induced preference for linear polarization $(\theta=0$ or $\pi$ ) in the absence of magnetic fields (see Chapter 6). The quantities $I_{\perp}$ (intensity of electric-field component perpendicular to $\mathrm{H}$ ), I// (intensity of parallel component), $X$ (ellipticity of the mode) and $U$ (azimuth of the mode, i.e., the angle between the long axis of the polarization ellipse and the applied magnetic field) are related to $I, R$ and $\theta$ in a simple way. One has [see also (2-35)]

$$
\begin{aligned}
& I_{\perp}=I \sin ^{2} R ; \quad I_{/ /}=I \cos ^{2} R ; \\
& \sin 2 x=\sin 2 R \sin \theta ; \tan 2 u=\tan 2 R \cos \theta .
\end{aligned}
$$

In the dispersion-free case of Fig.7.2a $(s=0)$, stable time-independent states of polarization are found for all values of the magnetic-field parameter $h$. In Fig.7.2b, with $s \neq 0$, a time-dependent state of polarization 


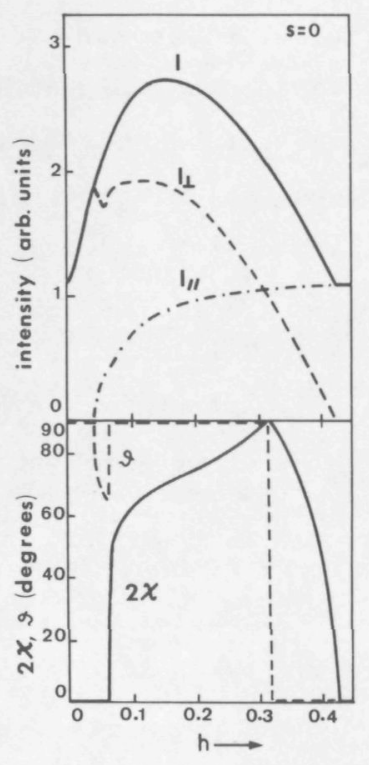

(a)

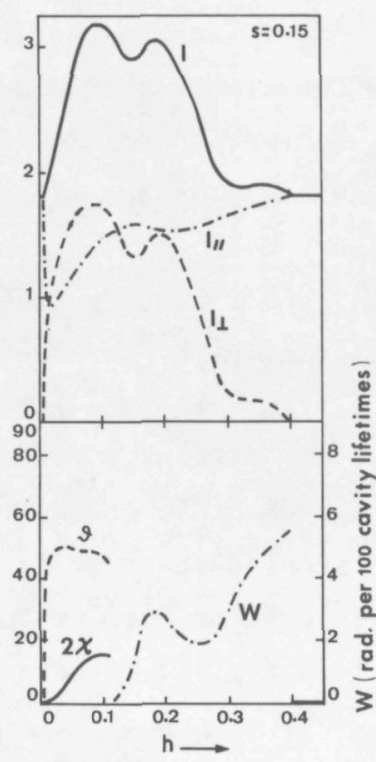

(b)

Fig.7.2 Mode-polarization parameters characterizing the (timeindependent) state of polarization of the laser mode, as a function of the Doppler-normalized magnitude $h$ of the applied transverse magnetic field. The figures refer to a $j_{\mathrm{a}}=1 \rightarrow j_{\mathrm{b}}=2$ type of transition, for two values of the detuning parameter $s$. $I_{\perp}$ is the intensity of the electric-field component perpendicular to the magnetic field; $I_{/ /}$is the intensity of the parallel component; $x$ is the ellipticity of the mode and $u$ its azimuth. I is the total intensity. $W$ is the difference frequency between the two electric-field components; it has been calculated by using $(D-4)$. The line width parameters are $\lambda=0.0287 ; \lambda_{a}=0.0178 ; \lambda_{b}=0.0396$. The excitation density $n_{0}=\alpha^{\prime \prime}(s=0, h=0) / \Gamma^{\prime}$ is taken equal to 1.2 . Only one of the two stable, time-independent solutions is drawn, the other one having opposite $x$ and $u$.

is found only for small $h$, whereas in the region of moderate magnetic fields a difference frequency $W \neq 0$ appears.

Many features of the curves of Fig.7.2 are well known by now [41] and need not be discussed here again. However, a few remarks should be made, 
concerning the results in weak magnetic fields. First, it should be mentioned that the behaviour of the laser mode in the weak-field region at nonzero detunings, as represented in Fig.7.2b turns out to depend critically on the relative magnitudes of the line widths. Calculations with $\lambda_{>} \pi_{a}, \pi_{b}$ predicted a behaviour of $I_{\perp}$ and $I_{/ /}$, similar to that shown in Fig.7.2a, even if $s \neq 0$. Second, the intensity curves of Fig.7.2a can be compared with the result of a calculation by Sargent et al. (Fig.13 of Reference [41]), since in our calculations the same line width parameters and excitation density have been taken. Except for the region of weak magnetic field, good quantitative agreement is found. The deviation between the results of our study and that of Sargent et al. is very probably due to an erroneous continuation of the mean-value approximation for $\sin 2 \theta$ and $\cos 2 \theta$, down to the weak-field region, whereas we have taken into account the phaselocking effect at small values of $h$.

In order to get an idea of the applicability of the approximative method, used in the $h$-region of Zeeman splitting close to the natural line widths, i.e. $|h| \simeq \lambda_{a}, \lambda_{b}$, equations $(7-1)-(7-3)$ have been numerically integrated for such a special case. The result is shown in Fig.7.3, where the time evolutions of $I, R$ and $\theta$ are plotted for some arbitrarily chosen starting values at $t=0$. For comparison, the corresponding time-independent values of $I$ and $R$, and the phase angle $\theta=W t$, calculated by means of the approximative method, are also indicated in the figure.

When comparing our results with the experiments reported by several authors $[13,22,43]$, there appears to be a discrepancy. For instance, in an almost isotropic He-Ne $1.152 \mu \mathrm{m}$ single-mode gas laser, subject to a transverse magnetic field of moderate and large magnitude, De Lang \& Bouwhuis [22] observed the laser field to be linearly polarized, while the main effect of the magnetic field was on the orientation of the polarization plane only. This observation was independent of the detuning frequency of the 1aser. An explanation of this apparent discrepancy between theory and experiment is given in the next section, where it is argued that the observation of De Lang \& Bouwhuis should indeed be expected when the active medium is only partly subject to a transverse magnetic field, while the other part is magnetic-field free. The discrepancies between the present results and the experimental observations of References [13] and [43] 


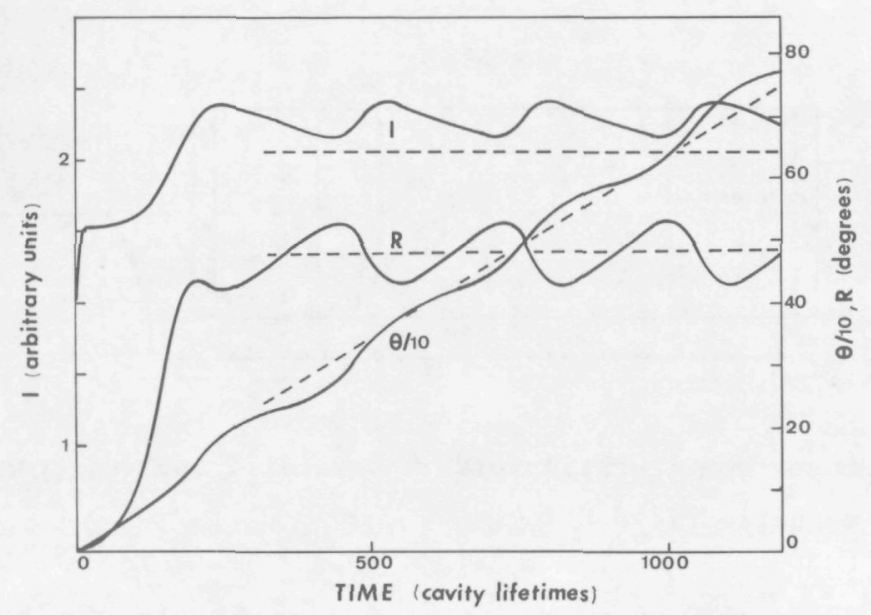

Fig.7.3 Transient time evolutions of $I, R$ and $\theta$, obtained by numerical integration of (7-1)-(7-3), for $s=0.05, h=0.04$, and some arbitrary starting condition at $t=0$. Type of transition, line widths and excitation density as in Fig.7.2. The straight (broken) lines indicate the values of $I, R$ and $\Theta=W t$, obtained by means of the approximative method used in the calculations of Fig.7.2

very probably have their origin in initial cavity anisotropies, as will be discussed in Section 7.3 .

\section{2 transverse magnetic field applied to a fraction of the resonator (isotropic loss tensor)}

The configuration to be studied is sketched in Fig.7.4. A uniform magnetic field $\underline{H}$ is transversally applied to the active medium over a fraction $f$ of the total resonator length $L$. The other part of the active medium is magnetic-field free. The resonator-induced contributions to the time evolutions of $I, R$ and $\theta$ are the same as in the previous analysis, whereas the medium-induced contributions now consist of two terms, with 


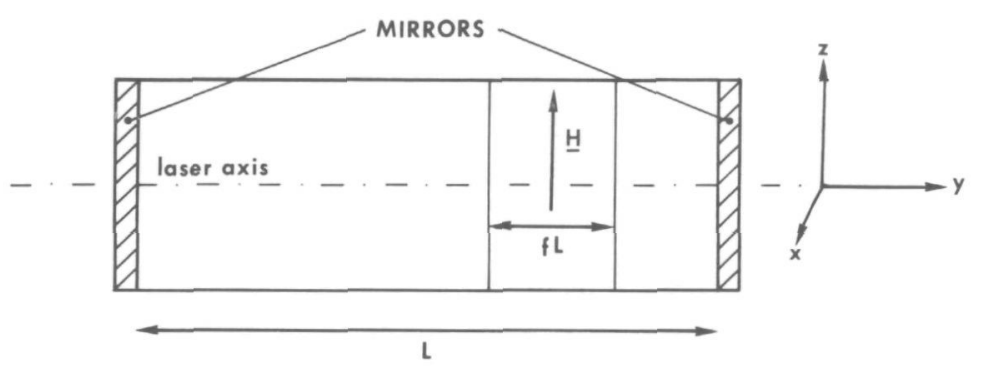

Fig.7.4 Laser configuration with a partially applied transverse magnetic field $\underline{H}$

fractions $f$ and $(1-f)$, respectively, in front. The first term is due to the layer of medium subject to the H-field; the second is due to the field-free part of the medium. Hence, using (2-60)-(2-62), first taken at $h \neq 0$ and, second at $h=0$, the expressions for the time evolutions of the polarization parameters can easily be obtained and need not be reproduced here.

As in the previous case, the equation for $a_{t} \theta$ determines whether the state of polarization will become time-independent or time-dependent. Its right-hand side now consists of two terms, the action of each being well understood. For instance, limiting ourselves to the $j_{\mathrm{a}}=1 \rightarrow j_{\mathrm{b}}=2$ type of transition, the magnetic-field-free part would lead, in the absence of the other term, to a stationary state with $\theta=0$ or $\theta=\pi$ (this corresponds to linear polarization), whereas the $h$-dependent term would lead, if existing alone, to the time-dependent polarization behaviour analyzed in the previous section, at least for nonzero detuning and for not too small values of $h$.

A stability analysis shows that, as long as the fraction $f$ is not too close to unity, the $d_{t} \ominus$ equation still admits (bi)stable time-independent solutions for $\theta$, for all values of $s$ and $h$. This is demonstrated in Fig.7.5, where for the same transition as in Fig.7.2 the polarization parameters have been plotted as a function of the magnetic-field parameter $h$, while the magnetic field is only present over a fraction $f=0.3$ of the active medium. Time-independent solutions, corresponding to nearly 1 inear polarization of the laser mode, are found for all values of $h$ and $s$. 


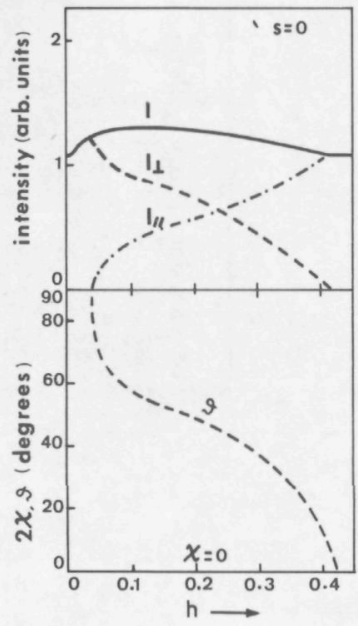

(a)

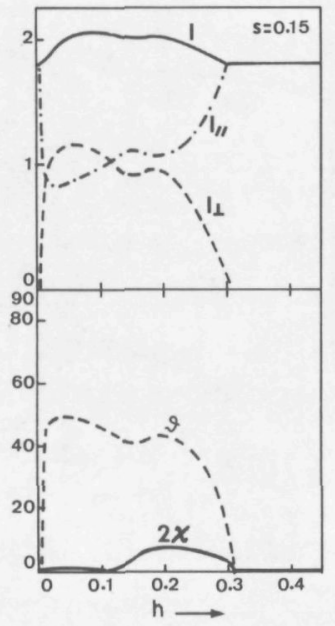

(b)

Fig.7.5 Mode-polarization parameters as a function of the Dopplernormalized magnitude $h$ of the applied transverse magnetic field. Only a fraction $f=0.3$ of the active medium is subject to the applied field. Other parameters are the same as in Fig.7.2. The polarization parameters corresponding to the other stable solutions, with opposite $x$ and $v$, have not been drawn

The intensities in Fig.7.5 at moderate values of $h$ are smaller than the corresponding values in Fig.7.2, but qualitatively they show the same behaviour. This is not the case for, the ellipticity, which is seen to be close to zero in all cases.

According to the plots of the azimuth $U$, the (bi)stable laser field stands under an angle between $40^{\circ}$ and $50^{\circ}$ with the applied field over a rather large region of $h$-values. At larger values of $h$, the long axes of the polarization ellipses move towards the direction of $\underline{H}$, coinciding there at large enough $h$, the mode being exactly linear then. These are the kind of effects observed by De Lang \& Bouwhuis [22]. 


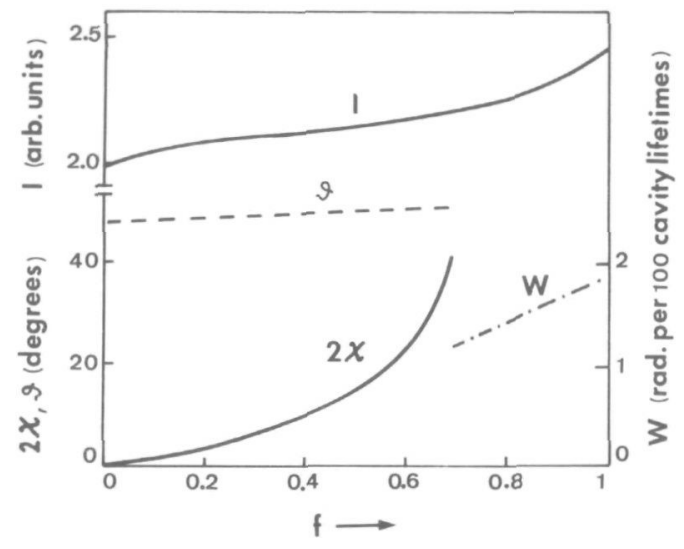

Fig.7.6 Mode-polarization parameters as a function of the fraction $f$, over which a transverse magnetic field $(h=0.15)$ is present. Detuning parameter $s=0.1$; 1 ine width parameters $\lambda=0.1$; $\lambda_{a}=0.02 ; \lambda_{b}=0.04$. The type of transition is $j_{a}=1 \rightarrow j_{b}=2$; The excitation density $n_{0}=1.2$. In the time-independent state there are two stable solutions only one of which has been indicated, the other having opposite $x$ and $u$.

Fig.7.6 shows the dependence of the polarization parameters on the fraction $f$, for a $j_{\mathrm{a}}=1 \rightarrow j_{\mathrm{b}}=2$ type of transition, at fixed values of nonzero detuning and magnetic-field magnitude. The mode is seen to become more and more elliptically polarized with increasing $h$, but the azimuth hardly changes at all. Time-independent states are found up to fno.7. At larger values of $f$ the preference for linear polarization in the magnetic-fieldfree region can no longer compete with the magnetic-field-induced, dispersive effects, which results in a time-dependent state with nonzero difference frequency.

The result of a calculation on a $j_{a}=j_{b}=2$ type of transition is shown in Fig.7.7. As we have seen in the preceding chapter, this transition shows a saturation-induced preference for circular polarization $(\chi= \pm \pi / 4)$, in the absence of any magnetic field. With increasing magnitude of the magnetic field, the ellipticity decreases monotonically until $h$ enters 


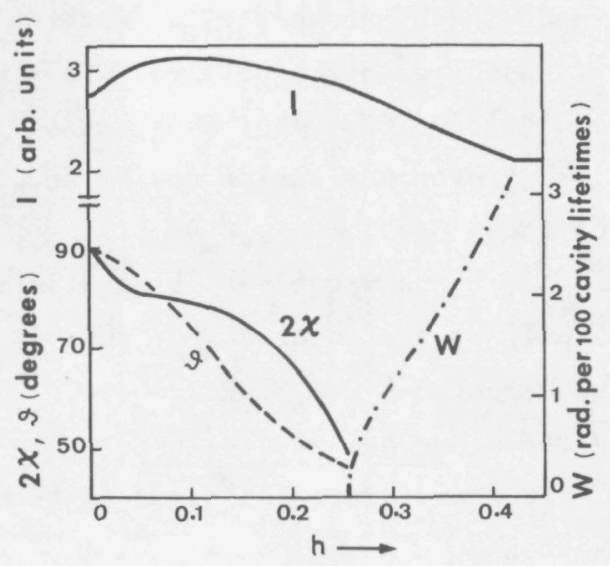

Fig.7.7 Mode-polarization parameters as a function of the magnitude of the magnetic field, applied to a fraction $f=0.5$ of the active medium. The transition is a $j_{\mathrm{a}}=2 \rightarrow j_{\mathrm{b}}=2$ type, showing circular polarization $(x= \pm \pi / 4)$ at $h=0$. Other parameters are as in Fig. 7.6

a region in which no stable, time-independent states are found, and a difference frequency appears. As is to be expected, smaller values of $f$ result in a narrowing, or even a disappearance, of this region.

\section{3 the influence of weak cavity anisotropies}

In general, the combined effect on the mode polarization due to the cavity anisotropy and the medium-induced anisotropy can lead to a complicated polarization behaviour of a laser mode. As in practice two different cavities are very unlikely to possess the same anisotropy, one should not be surprised to observe that two apparently equal gas lasers, which operate on the same type of transition, exhibit a different polarizing behaviour. As will be pointed out below, the discrepancy between the experimental observations of References [13] and [43]might be an example of this. 
Let us first consider the experiment of Dumont \& Durand [13]. The observed bistability of the mode in the absence of an external magnetic field confirms that the cavity used in this experiment was very likely to possess an 1.p.a. (see a1so Section 6.3). For this case, with a transversally applied magnetic field $\underline{H}$ over the entire cavity and oriented along one of the main axes of the 1.p.a. [i.e., $\theta_{p}=0$ or $\theta_{p}=\pi / 2$ in $\left.(B-4)\right]$, the differential equations for $I, R$ and $\theta$ can easily be written down. They differ from (7-1)-(7-3) only in the equation for $a_{t} \theta$, in which the right-hand side has to be supplemented by $\mp_{p} \Gamma_{p} \sin 2 R$, where $\Gamma_{p}$ is the magnitude of the 1.p.a., and the minus and plus sign refer to $\theta_{p}=0$ and $\Theta_{p}=\pi / 2$, respectively. Hence, after dividing both sides of the modified equation (7-2) by $\sin 2 R$, we obtain

$$
\begin{aligned}
d_{t} \Theta=\mp \Gamma_{p}+\alpha_{1}^{\prime} / 2+ & I\left(\gamma^{\prime \prime} \sin 2 \Theta+\gamma^{\prime} \cos 2 \theta \cos 2 R-\gamma_{1}^{\prime} \sin 2 \theta \cos 2 R\right. \\
& \left.-\gamma_{1}^{\prime} \cos 2 \Theta-\beta_{2}^{\prime}-\delta^{\prime} \cos 2 R\right),
\end{aligned}
$$

which makes sense only if $R \neq 0$ and $R \neq \pi / 2$.

Since the difference frequency, observed by Dumont and Durand at moderate applied fields, did not depend on the magnitude of the magnetic field, the contribution of the 1.p.a. to the right-hand side of (7-5) should be an order of magnitude larger than the other terms. Therefore, we can take as an approximation $d_{t} \Theta=\mp \Gamma$. As a further approximation, we will take the rapidly varying terms proportional to $\sin 2 \theta$ and $\cos 2 \theta$ equal to zero in the equations for $d_{t} R$ and $d_{t} I$, which then read

$$
\begin{aligned}
&(1 / I) \mathrm{d}_{t} I=\alpha^{\prime \prime}-\Gamma^{\prime}-\alpha_{1}^{\prime \prime} \sin ^{2} R \\
&-I\left\{\beta^{\prime \prime}-\left(\beta_{1}^{\prime \prime}+\delta^{\prime \prime} \cos ^{2} R\right) \sin ^{2} R\right\} ; \\
& 2 \mathrm{~d}_{t} R=\sin 2 R\left\{-\alpha_{1}^{\prime \prime} / 2+I\left(\beta_{2}^{\prime \prime}+\delta^{\prime \prime} \cos 2 R\right)\right\} .
\end{aligned}
$$

The time-independent solutions are: 
(a) $R=0 ; \quad I=\frac{\alpha^{\prime \prime}-\Gamma^{\prime}}{\beta^{\prime \prime}}$,

if $I\left(\delta^{\prime \prime}+\beta_{2}^{\prime \prime}\right)<2 \alpha_{1}^{\prime \prime}$, corresponding to linear polarization of the mode along the magnetic field ( $\pi$-polarization);

(b) $R=\pi / 2 ; \quad I=\frac{\alpha^{\prime \prime}-\alpha_{1}^{\prime \prime}-\Gamma^{\prime}}{\beta^{\prime \prime}-\beta_{1}^{\prime \prime}}$,

if $I\left(\delta^{\prime \prime}-\beta_{2}^{\prime \prime}\right)<-2 \alpha_{1}^{\prime \prime}$, corresponding to linear polarization of the mode perpendicular to the magnetic field ( $\sigma$-polarization);

(c) $\cos 2 R=\left(2 \alpha_{1}^{\prime \prime} / I-\beta_{2}^{\prime \prime}\right) / \delta^{\prime \prime}$;

$$
I=\frac{\alpha^{\prime \prime}-\Gamma^{\prime}-\alpha_{1}^{\prime \prime}\left(\delta^{\prime \prime}+2 \beta_{1}^{\prime \prime}-\beta_{2}^{\prime \prime}\right) / 2 \delta^{\prime \prime}}{\beta^{\prime \prime}-\left\{\left(\delta^{\prime \prime}+\beta_{1}^{\prime \prime}\right)^{2}-\left(\beta_{1}^{\prime \prime}-\beta_{2}^{\prime \prime}\right)^{2}\right\} / 4 \delta^{\prime \prime}},
$$

if both (a) and (b) are unstable, corresponding to a timedependent state of polarization with difference frequency $W^{h} \Gamma_{p}$.

For the $j_{\mathrm{a}}=1 \rightarrow j_{\mathrm{b}}=2$ type transition, which is the transition in the experiment of Dumont \& Durand, one has, if there is no external field, $\alpha_{1}^{\prime \prime}=\beta_{2}^{\prime \prime}=0$ and $\delta^{\prime \prime}<0$. Therefore, with no field applied, both solutions (a) and (b) are stable. With nonzero applied magnetic field, our calculations show that four successive magnetic-field regions can be distinguished, each of which corresponds with a particular polarization behaviour. This is indicated in Fig.7.8, where the intensities of the laser-field components, parallel and perpendicular, respectively, to the magnetic field are plotted as a function of its Doppler-normalized magnitude $h$. The bistable region $h<h_{1}$ is followed by a region $h_{1}<h<h_{2}$, in which solution (b) is the only stable one, while in the region $h_{2}<h<h_{3}$ the mode is in the time-dependent state given by solution (c). For $h>h_{3}$ the mode is polarized along the magnetic field [solution (a) again]. It should be noticed that these results do not depend on the particular sign of the 1.p.a.-term in $(7-5)$. Hence, a rotation of the magnetic field over $\pi / 2$ does not change the theoretical results. These are precisely the effects reported in Reference [13]. 


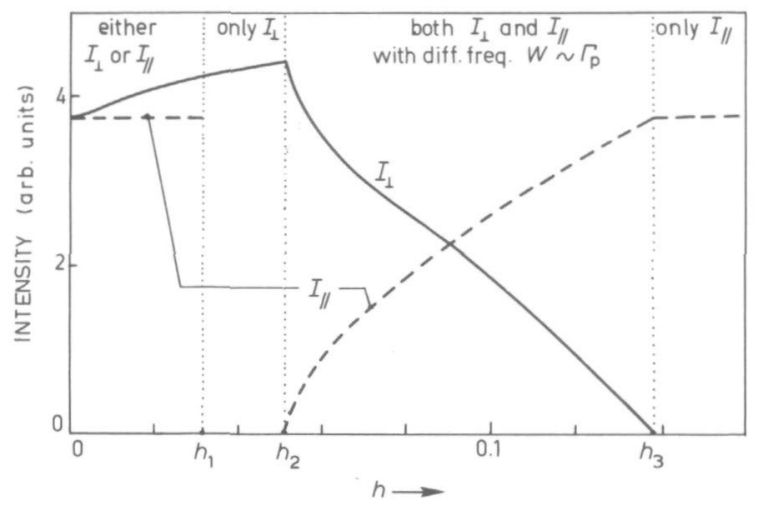

Fig.7.8 Intensity curves versus Doppler-normalized magnetic-field magnitude $h$ of the applied, transverse magnetic field. $I_{\perp}$ is the intensity of the electric-field component perpendicular to the applied field; $I_{/ /}$is the intensity of the parallel component. A linear phase anisotropy has been assumed, the magnitude of which should be such as to overrule all other terms in the r.h. side of (7-5). This turns out to be the case whenever $\Gamma_{p}>0.7 \%$ of the isotropic cavity loss $\Gamma^{\prime}$. One of the main axes of the 1.p.a. is oriented along the magnetic field. The parameters are $f=1 ; j_{\mathrm{a}}=1 ; j_{\mathrm{b}}=2 ; n_{0}=1.04$; $s=0 ; \lambda=0.1 ; \lambda_{a}=0.02 ; \lambda_{b}=0.04$

A quite different behaviour is observed in the experiment of Settles \& Heer [43], where a rotation over $\pi / 2$ of the transversally applied magnetic field did drastically change the observations in the region of small fields. In this experiment the resonator was therefore more likely to possess a linear amplitude anisotropy (1.a.a.) than an 1.p.a. As will be shown below, the assumption of a small 1.a.a. is sufficient to explain the results of Settles \& Heer.

With a transverse magnetic field applied in the z-direction over the entire cavity, and with an 1.a.a. of magnitude $\Gamma_{a}$, whose main axis corresponding to low loss is either parallel or perpendicular to the magnetic 


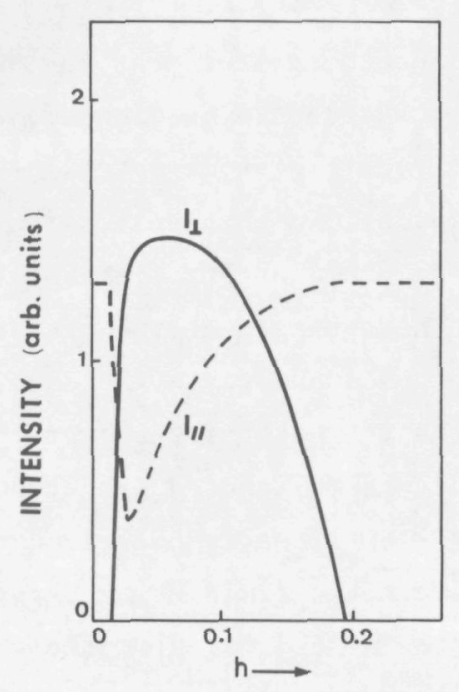

(a)

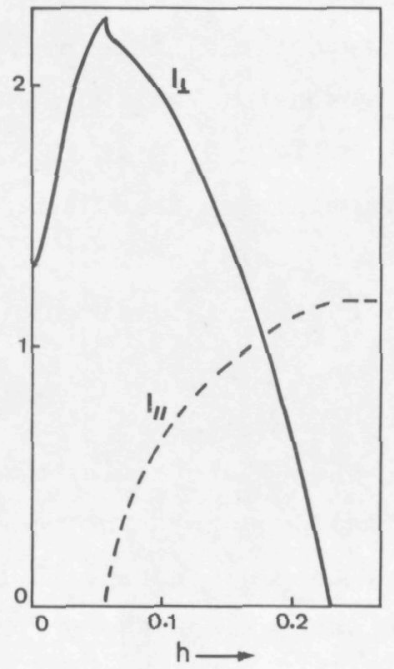

(b)

Fig.7.9 Intensity curves versus magnetic-field parameter $h$ for two orientations of the transverse magnetic field with respect to the initial resonator anisotropy. A linear amplitude anisotropy of magnitude $\Gamma_{\mathrm{a}}=0.5 \%$ of the isotropic loss $\Gamma^{\prime}$ has been assumed. In (a) the magnetic field is along the lowloss main axis; in (b) it is along the high-loss main axis. Other parameters are $f=1 ; j_{a}=1 ; j_{b}=2 ; n_{0}=1.05 ; s=0 ; \lambda=0.0287$; $\lambda_{a}=0.0178 ; \lambda_{b}=0.0396$

field, i.e., $\theta_{a}=0$ or $\theta_{a}=\pi / 2$, respectively, in $(B-9)-(B-11)$, the equations describing the time evolution of the polarization parameters are given by $(7-1)-(7-3)$, extended with the term $\Gamma_{\mathrm{a}} \cos 2 R$ in the right-hand side of analum (7-3) and with $\mp \Gamma_{a} \sin 2 R$ in the right-hand side of (7-1). The upper and lower signs correspond to $\theta_{a}=0$ and $\theta_{a}=\pi / 2$, respectively. The results of a numerical stability analysis applied to the thus adapted aquations for a $j_{\mathrm{a}}=1 \rightarrow j_{\mathrm{b}}=2$ type transition for central tuning $(s=0)$ are presented in Fig.7.9. The laser field components parallel and perpendicular, respectively, to the applied field are plotted as a function of $h$. Fig.7.9a refers to the case in which the magnetic field is parallel to the low-loss 
main axis of the 1.a.a. $\left(\theta_{a}=0\right)$. In Fig.7.9b the field has been rotated over $\pi / 2\left(\theta_{\mathrm{a}}=\pi / 2\right)$.

To understand the differences, we should realize that in the case of an isotropic resonator the two pictures would have been equal, looking very similar to Fig.7.9b with the $I_{/ /}$curve 1ying somewhat higher and the $I_{\perp}$ curve somewhat lower (see also Fig.7.2a). In that case, with a small nonzero magnetic field applied, the mode would prefer linear polarization, perpendicular to the field direction, the strength of the preference being, roughly speaking, proportional to $h^{2}$. Consequently, in the case of Fig.7.9a, with $\underline{H}$ along the low-loss main axis, the resonator-induced preference will overrule the counteracting magnetic-field-induced preference. That is to say, for small enough $h$ the mode will be linearly polarized along the applied field, the only effect of the magnetic field being a reduction in the effective strength of the preference. As the magnetic-field-induced anisotropy rapidly grows with increasing $h$, it will soon overrule the effect of the 1.a.a., which results in a rapid recovery of the situation that would have occurred in the isotropic-resonator case. In the case of Fig.7.9b, both the magnetic-field-induced and resonator-induced anisotropy tend to the same preference for small $h$, that is, linear polarization of the mode, perpendicular to the field direction. These results are in good qualitative agreement with the observations of Settles \& Heer.

\section{4 polarization effects in large transverse magnetic fields}

The polarizing effect on the output of a gas laser due to the application of a transverse magnetic field of sufficiently large magnitude to (fractions of) the active medium, offers a simple method to obtain a high degree of "linear" polarization of the laser light $[46,48,49]$. Roughly speaking, the magnitude of the magnetic field should be so large that the Zeeman splitting exceeds the width of the gain profile, in order to assure that only $\pi$-transitions (with polarization paralle1 to $\underline{H}$ ) contribute to the laser field. In the Doppler limit, the condition for this reads

$$
\frac{1}{2}\left\{e^{-(s-h)^{2}}+e^{-(s+h)^{2}}\right\}<n_{0}^{-1},
$$


where the excitation density $n_{0}=\alpha^{\prime \prime}(s=0, h=0) / \Gamma^{\prime}$. It is assumed that the magnetic field acts over the entire resonator. In practice, small deviations of condition (7-11) should be expected, since the influence of saturation effects are neglected. As an example, in a single-mode $0.6328 \mu \mathrm{m}$ He-Ne gas laser with excitation density $n_{0}=1.2$, condition (7-11) will be satisfied for all $s$ whenever $h>0.43$, which corresponds to magnetic fields $\mathrm{H}>0.024 \mathrm{~T}$.

Due to small anisotropies in the resonator loss, the laser field will not be exactly linearly polarized at these magnetic-field values. In this respect we refer to the studies by Crisp [48] and by Keijser [49]. In this section we will theoretically investigate such effects. Let us start by giving a (semi-) quantitative approach. Suppose that a transverse magnetic field $\underline{H}$ (along the z-axis) is present, while its magnitude is so large that condition (7-11) is satisfied. If we assume for the moment that a linearly polarized electric field with complex amplitude $E_{z}$ and real frequency $\omega$ is present, then it is obvious that, due to the resonator anisotropy, a small orthogonal component with complex amplitude $E_{x}$ will be created, whose rate follows from the laser equation (2-26) and satisfies

$$
d_{t} E_{x}+\frac{1}{2}\left(\Gamma_{x x} E_{x}-i \frac{\omega}{\varepsilon_{0}} P_{x}\right)=-\frac{1}{2} \Gamma_{x z} E_{z} .
$$

Since $\left|E_{x}\right|$ is small, the terms in $P_{x}$ which depend nonlinearly on $E_{x}$ can be ignored. The equation for $E_{x}$ can then be written as

$$
\mathrm{d}_{t} E_{x}+\frac{1}{2}\left(\Gamma_{x x}+i a_{x}\right) E_{x}=-\frac{1}{2} \Gamma_{x z} E_{z},
$$

where $a_{x}$ is given by [see (2-59) and (C-2)]

$$
a_{x}=\frac{\omega \overline{\mathrm{N}} p^{2} E_{1}}{2 \varepsilon_{0} \hbar \lambda_{\mathrm{d}}}\left\{J_{0}(s+h)+J_{0}(s-h)\right\} .
$$

If the laser is oscillating in a stationary state, the solution of (7-13) is

$$
\frac{E_{x}}{E_{z}}=-\frac{\Gamma_{x z}}{\Gamma_{x x}+i a_{x}} .
$$


By substitution of the general expressions for $\Gamma_{x z}$ and $\Gamma_{x x}$ (Appendix B) into (7-15), we find for the corresponding intensity ratio

$$
\frac{I_{x}}{I_{z}}=\frac{\left(\Gamma_{\mathrm{a}} \sin 2 \theta_{\mathrm{a}}+\Gamma_{\mathrm{c}}^{\prime \prime}\right)^{2}+\left(\Gamma_{\mathrm{p}} \sin 2 \theta_{p}-\Gamma_{c}^{\prime}\right)^{2}}{\left(\Gamma^{\prime}+\Gamma_{\mathrm{a}} \cos 2 \theta_{\mathrm{a}}-a_{x}^{\prime \prime}\right)^{2}+\left(\Gamma^{\prime \prime}+\Gamma_{p} \cos 2 \theta_{p}+a_{x}^{\prime}\right)^{2}} .
$$

It is seen that rotation of the resonator around its optical axis, while the magnetic field is kept fixed, generally causes the right-hand side of (7-16) to oscillate with a period of $\pi$. Only in special cases, for instance if $\Gamma_{c}=0$ and $\Gamma_{a}=0$ or $\Gamma_{p}=0$, do orientations of the resonator with respect to the magnetic field, for which $I_{x}=0$, exist. In practice, however, such cases are very unlike1y.

If the magnetic-field magnitude is taken so large that $\Gamma^{\prime}-a_{x}^{\prime \prime}>\Gamma_{a}$, the denominator in the right-hand side of (7-16) is practically independent of the orientation of the resonator with respect to $\underline{H}$. In that case (7-16) reduces to the simple formula

$$
\frac{I_{x}}{I_{z}}=\text { Const. }\left\{\left(\Gamma_{\mathrm{a}} \sin 2 \theta_{\mathrm{a}}+\Gamma_{\mathrm{c}}^{\prime \prime}\right)^{2}+\left(\Gamma_{\mathrm{p}} \sin 2 \theta_{\mathrm{p}}-\Gamma_{\mathrm{c}}^{\prime}\right)^{2}\right\} .
$$

In a more sophisticated approach, we have to solve numerically the appropriate equations for the polarization parameters for the case in which the absence of a resonator anisotropy would lead to linear polarization of the laser mode along the direction induced by the magnetic field. The equations to be used are (7-1)-(7-3), the right-hand sides of which have to be supplemented by the resonator-induced contributions (B-9)-(B-11). In Fig.7.10 the results are given for a configuration involving a transverse magnetic field applied to a fraction $f$ of the active medium (see Fig.7.4). For a number of fractions varying from $f=0.2$ to $f=1$, the ratio $I_{x} / I_{z}$ is plotted as a function of the orientation of one of the anisotropy main axis with respect to the direction of $\underline{H}$. The broken lines represent the results obtained with (7-17) if for each $f$ the constant is chosen such that the absolute maxima of corresponding curves coincide. We conclude that the angular dependence of $I_{x} / I_{z}$ is reasonably well described by the qualitative formula (7-17). 


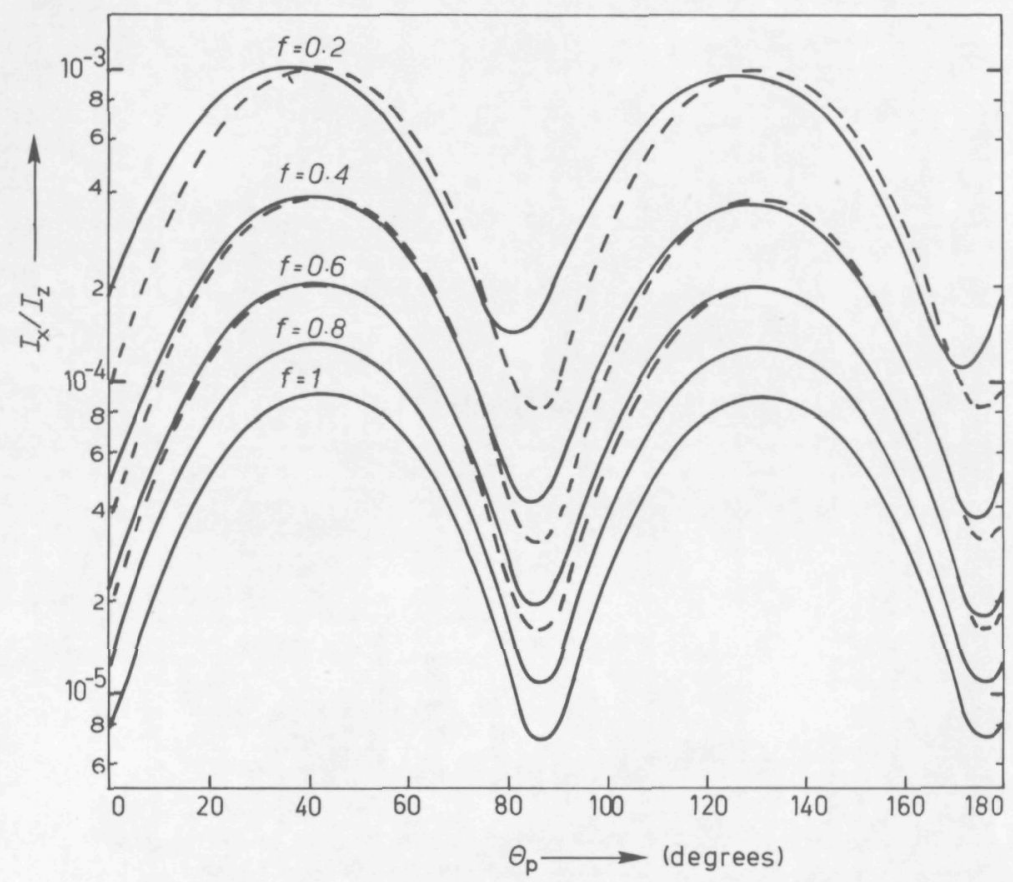

Fig.7.10 Effect of a small resonator anisotropy on the output of a single-mode gas laser, polarized by means of a transverse magnetic field acting over a fraction $f$ of the resonator length. The quantity along the vertical axis is the ratio $I_{x} / I_{z}$ of the intensities corresponding to the electric-field components perpendicular and parallel to the magnetic field, respectively. The anisotropy has been taken such that $\Gamma_{a}=$ $0.005 \Gamma^{\prime} ; \Gamma_{p}=0.01 \Gamma^{\prime} ; \theta_{a}-\theta_{p}=20^{\circ} ; \Gamma_{c}^{\prime}=\Gamma_{c}^{\prime \prime}=0$. The quantity along the horizontal axis is the angle $\Theta_{p}$ between the "slow" axis of the 1.p.a. and the magnetic field. The magnitude of the applied field is such as to give rise to linear polarization of the mode along the applied field in the isotropic cavity case. The broken lines represent the results obtained with (7-17) if for each $f$ the constant is chosen such that the absolute maxima of corresponding curves coincide. The parameters are $s=0 ; j_{a}=1 ; j_{b}=2 ; n_{0}=1.3 ; \lambda=0.1 ; \lambda_{a}=0.02 ; \lambda_{b}=0.04$ 


\section{APPEND I CES}

\section{A. calculation of natural-modes in the one-dimensional model}

For the model described in Section 2.3 we wish to prove that in the region between the two mirrors the natural-modes are given by (2-13). It is evident that

$$
\underline{E}(n, t)=e^{-i \omega t} \underline{u}(n)+c \cdot c .
$$

fulfils the wave equation in free space if $\underline{u}(n)$ is of the form

$$
\underline{u}(n)=\frac{1}{2 i}\{\underline{A}(n)+\underline{B}(n)\} \equiv \frac{1}{2 i}\left\{\underline{a} \mathrm{e}^{i \omega n / c}+\underline{b} \mathrm{e}^{-i \omega n / c}\right\},
$$

where the (generally complex) amplitude vectors $\underline{a}$ and $\underline{b}$ are independent of $n$. From the boundary conditions, first in $n=0$ and, second in $n=L$, it follows that

$$
\underline{b}=-\underline{a},
$$

and

$$
\underline{\underline{S}}(\omega) \underline{a}=e^{-2 i \omega L / c} \underline{a},
$$

where $\underset{\underline{S}}{S}(\omega)$ is the reflection matrix introduced in Section 2.3. If the mirror at $n=L$ is partially transmittant, the factor $\exp (-2 i \omega L / c)$ in the right-hand side of (A-4) will have absolute value smaller than one. Consequently, the imaginary part of $\omega$ is negative in such a case.

For each $\omega$, the reflection matrix $\underline{\underline{S}}(\omega)$ has two eigenvectors, which we will denote by $\underline{\sigma}_{q}(\omega)$, where $q=1,2$. The eigenvalue equation can generally be written as 


$$
\underline{\underline{S}}(\omega) \underline{\sigma}_{q}(\omega)=\mathrm{e}^{-(2 L / c)\left\{\lambda_{q}(\omega)-i \phi_{q}(\omega)\right\}} \underline{\sigma}_{q}(\omega),
$$

where $\lambda_{q}(\omega)$ and $\phi_{q}(\omega)$ are real numbers. By comparing (A-4) with (A-5), we obtain the frequency-determining equation

$$
\mathrm{e}^{-2 i \omega L / c}=\mathrm{e}^{-(2 L / c)\left\{\lambda_{q}(\omega)-i \phi_{q}(\omega)\right\}},
$$

or,

$$
\omega=\frac{n \pi c}{L}-\phi_{q}(\omega)-i \lambda_{q}(\omega),
$$

for $q=1,2$ and $n=0,1,2, \ldots$ Equation $(A-7)$ has to be solved for $\omega$. If we assume that $\lambda_{q}(\omega)$ and $\phi_{q}(\omega)$ depend only weak1y on $\omega$, which is always true in practical cases, (A-7) has for each combination $n, q$ precisely one solution, which we will denote by

$$
\omega_{n, q}^{c}=n \pi c / L-\phi_{n, q}-i \lambda_{n, q},
$$

where, in first approximation,

$$
\lambda_{n, q}=\lambda_{q}\left(\frac{n \pi c}{L}\right) ; \quad \phi_{n, q}=\phi_{q}\left(\frac{n \pi c}{L}\right) .
$$

The corresponding natural-modes, consistent with the boundary conditions $(A-3)$ and $(A-4)$, are thus given by

$$
\begin{array}{r}
\underline{u}_{n, q}(n)=\frac{1}{2 i}\left\{e^{\frac{1}{c}\left(\lambda_{n, q}-i \phi_{n, q}+i n \pi c / L\right) n}\right. \\
\left.-e^{-\frac{1}{c}\left(\lambda_{n, q}-i \phi_{n, q}+i n \pi c / L\right) n}\right\} \sigma_{n, q},
\end{array}
$$

where

$$
\underline{\sigma}_{n, q}=\underline{\sigma}_{q}\left(\omega_{n, q}^{c}\right) \text {. }
$$


In practical cases where the model will be applied, $\lambda_{n, q}$ and $\phi_{n, q}$ are very small compared to the longitudinal mode spacing $\pi_{c} / L$. For instance, if we take $L=0.5 \mathrm{~m}$ and a mirror reflectivity of $98 \%$, we find $\lambda_{n, q} \sim 6 \mathrm{MHz}$, while $c / 2 L \sim 300 \mathrm{MHz}$. From laser experiments it can be estimated that for the usual applied mirrors $[39,49]$

$$
\left|\frac{\lambda_{n, 1}-\lambda_{n, 2}}{\lambda_{n, 1}+\lambda_{n, 2}}\right| \lesssim\left|\frac{\phi_{n, 1}-\phi_{n, 2}}{\lambda_{n, 1}+\lambda_{n, 2}}\right| \lesssim 510^{-2} .
$$

\section{$B$. the Loss tensor}

The loss tensor $\Gamma_{n}$ acting in (2-26) can be represented by a complex $2 \times 2$ matrix. According to the classification scheme in Appendix A of reference [39], each $\stackrel{\Gamma}{=}$ can uniquely be written as (omitting the mode index number $n$ )

$$
\Gamma=\sum_{q=1}^{4} \Gamma^{q},
$$

where the four components are, respectively, (1) the isotropic amplitude and phase loss; (2) the linear amplitude anisotropy (1.a.a.); (3) the linear phase anisotropy (1.p.a.); and (4) the circular anisotropy in amplitude and phase loss. In the $(\zeta, \xi)$ basis, the components $\stackrel{\Gamma}{q}^{q}$ are given by

$$
\stackrel{\Gamma}{=}=\Gamma\left(\begin{array}{ll}
1 & 0 \\
0 & 1
\end{array}\right),
$$

describing isotropic amplitude and phase loss ( $\Gamma$ is complex);

$$
\stackrel{\Gamma}{=}^{2}=-\Gamma_{a}\left(\begin{array}{cc}
\cos _{2} \theta_{a} & \sin 2 \theta_{a} \\
\sin 2 \theta_{a} & -\cos 2 \theta_{a}
\end{array}\right),
$$

with $\Gamma_{a}>0$ and $-\pi / 2<\theta_{a} \leq \pi / 2$, describing an $1 . a . a$. of magnitude $\Gamma_{a}$, whose 
main axes are oriented with respect to the $\zeta$-axis at angles $\Theta_{a}$ (low-loss axis) and $\theta_{a} \pm \pi / 2$ (high-loss axis), respectively;

$$
\stackrel{\Gamma^{3}}{=}=-i \Gamma_{p}\left(\begin{array}{cc}
\cos 2 \theta_{p} & \sin 2 \theta_{p} \\
\sin 2 \theta_{p} & -\cos 2 \theta_{p}
\end{array}\right),
$$

with $\Gamma_{p}>0$ and $-\pi / 2<\theta_{p} \leq \pi / 2$, describing an $1 . p \cdot a$. of magnitude $\Gamma_{p}$, whose main axes are oriented with respect to the $\zeta$-axis at angles $\theta_{p}$ (lowfrequency axis) and $\theta_{p} \pm \pi / 2$ (high-frequency axis), respectively;

$$
\Gamma^{4}=\Gamma_{c}\left(\begin{array}{cc}
0 & i \\
-i & 0
\end{array}\right) \text {, }
$$

where $\Gamma_{c}$ is a complex number, describing a circular anisotropy in amplitude and phase loss.

By using (2-26) and $(B-1)-(B-5)$, we can easily obtain the contributions to the right-hand sides of (2-48)-(2-50) due to the effect of the empty resonator. In terms of $x$ and $u$ (axial field case) we find

$$
\begin{aligned}
& \left.2 \partial_{t} x\right|_{\text {resonator }}=-\Gamma_{p} \sin 2\left(u-\Theta_{p}\right)-\Gamma_{a} \cos 2\left(u-\Theta_{a}\right) \sin 2 x+\Gamma_{c}^{\prime} \cos 2 x ; \\
& \left.2 \cos 2 x \partial_{t} u\right|_{\text {resonator }}=\Gamma_{p} \cos 2\left(u-\Theta_{p}\right) \sin 2 x-\Gamma_{a} \sin 2\left(u-\Theta_{a}\right)-\Gamma_{c}^{\prime \prime} \cos 2 x ; \\
& \left.I^{-1} \partial_{t} I\right|_{\text {resonator }}=-\Gamma^{\prime}+\Gamma_{a} \cos 2\left(u-\Theta_{a}\right) \cos 2 x+\Gamma_{c}^{\prime} \sin 2 x ;
\end{aligned}
$$

where $\Gamma_{c}^{\prime}$ is the real part of $\Gamma_{c}$ and $\Gamma_{c}^{\prime \prime}$ is the imaginary part. In terms of $R$ and $\theta$ (transverse field case) the resonator-induced contributions read

$$
\begin{aligned}
\left.2 \partial_{t} R\right|_{\text {resonator }}= & \Gamma_{\mathrm{a}}\left(\sin 2 \theta_{\mathrm{a}} \cos \theta \cos 2 R-\cos 2 \theta_{\mathrm{a}} \sin 2 R\right) \\
& +\Gamma_{\mathrm{p}} \sin 2 \theta_{\mathrm{p}} \sin \theta+\Gamma_{\mathrm{c}}^{\prime} \sin \theta \cos 2 R-\Gamma_{\mathrm{c}}^{\prime \prime} \cos \theta ; \\
\left.\sin 2 R \partial_{t} \Theta\right|_{\text {resonator }} & -\Gamma_{\mathrm{a}} \sin 2 \theta_{\mathrm{a}} \sin \theta+\Gamma_{\mathrm{p}}\left(\sin 2 \theta_{\mathrm{p}} \cos \theta \cos 2 R\right. \\
& \left.-\cos 2 \theta_{\mathrm{p}} \sin 2 R\right)+\Gamma_{\mathrm{c}}^{\prime \prime} \sin \theta \cos 2 R+\Gamma_{\mathrm{c}}^{\prime} \cos \theta ;
\end{aligned}
$$




$$
\begin{aligned}
\left.I^{-1} \partial_{t} I\right|_{\text {resonator }} & =-\Gamma^{\prime}+\Gamma_{\mathrm{a}}\left(\cos 2 \theta_{\mathrm{a}} \cos 2 R+\sin 2 \theta_{\mathrm{a}} \cos \theta \sin 2 R\right) \\
& +\Gamma_{\mathrm{c}}^{\prime} \sin \theta \sin 2 R
\end{aligned}
$$

C. calculation of the $\alpha, \beta, \gamma$ and $\delta$ coefficients

By comparing (2-40)-(2-42) with (5-57), taking $n=n^{\prime}=n^{\prime \prime}=n^{\prime \prime}$ in the latter equation, we easily find

$$
\begin{aligned}
& a_{j}=a_{j}^{n} ; b_{ \pm 1, \pm 1}=b_{ \pm 1 ; \pm 1, \pm 1, \pm 1}^{n ; n, n, n} ; b_{0,0}=b_{0 ; 0,0,0}^{n ; n, n, n} ; \\
& b_{ \pm 1, \mp 1}=b_{ \pm 1 ; 1, \pm 1, \mp 1}^{n ; n, n, n}+b_{ \pm 1 ; \mp 1, \mp 1, \pm 1}^{n ; n, n, n} ; \\
& b_{ \pm 1,0}=b_{ \pm 1 ; 0,0, \pm 1}^{n ; n, n, n}+b_{ \pm 1 ; 0, \pm 1,0}^{n ; n, n, n} ; \\
& b_{0, \pm 1}=b_{0 ; \pm 1, \pm 1,0}^{n ; n, n, n}+b_{0 ; \pm 1,0, \pm 1}^{n ; n, n, n} ; \\
& c_{0}=b_{0 ; 0,1,-1}^{n ; n, n, n}+b_{0 ; 0,-1,1}^{n ; n, n, n} ; c_{ \pm 1}=b_{ \pm 1 ; \mp 1,0,0}^{n ; n, n, n}
\end{aligned}
$$

Explicit expressions for the $a, b$ and $c$ coefficients are derived by using (5-54) and (5-55). If we evaluate the traces in (5-55) by using (5-37)-(5-41), we obtain the following result:

$$
\begin{aligned}
a_{\imath}=\frac{\Omega \bar{N} p^{2}}{\varepsilon_{0} \hbar \lambda_{d}} F_{1} J_{0}(s+Z h), \quad(Z=0, \pm 1) ; \\
b_{ \pm 1, \pm 1}=A F_{2}\left(1 / i \lambda_{a}+1 / i \lambda_{b}\right)\left\{J_{3}(s \pm h,-s \mp h)+J_{3}(s \pm h, s \pm h)\right\} ; \\
b_{ \pm 1, \overline{+1}}=A\left\{\left(\frac{F_{3}+F_{4}-F_{2}}{ \pm 2 h+i \lambda_{a}}+\frac{F_{3}-F_{4}}{ \pm 2 h+i \lambda_{b}}\right)\left\{J_{3}(s \pm h,-s \pm h)+J_{3}(s \pm h, s \pm h)\right\}\right. \\
\left.+\left(\frac{F_{3}-F_{4}}{i \lambda_{a}}+\frac{F_{3}+F_{4}-F_{2}}{i \lambda_{b}}\right)\left\{J_{3}(s \pm h,-s \pm h)+J_{3}(s \pm h, s \mp h)\right\}\right\}
\end{aligned}
$$




$$
\begin{array}{rl}
b_{0,0}= & A F_{3}\left(1 / i \lambda_{\mathrm{a}}+1 / i \lambda_{\mathrm{b}}\right)\left\{J_{3}(s,-s)+J_{3}(s, s)\right\} ; \\
b_{ \pm 1,0}= & A\left\{\left(\frac{F_{2}-F_{4}}{i \lambda_{\mathrm{a}}}+\frac{F_{4}}{i \lambda_{\mathrm{b}}}\right)\left\{J_{3}(s \pm h,-s)+J_{3}(s \pm h, s)\right\}\right. \\
& \left.+\left(\frac{F_{4}}{ \pm h+i \lambda_{\mathrm{a}}}+\frac{F_{2}-F_{4}}{ \pm h+i \lambda_{\mathrm{b}}}\right)\left\{J_{3}(s \pm h,-s)+J_{3}(s \pm h, s \pm h)\right\}\right\} ; \\
b_{0, \pm 1} & A\left\{\left(\frac{F_{2}-F_{4}}{i \lambda_{\mathrm{a}}}+\frac{F_{4}}{i \lambda_{\mathrm{b}}}\right)\left\{J_{3}(s,-s \mp h)+J_{3}(s, s \pm h)\right\}\right. \\
& \left.+\left(\frac{F_{4}}{\overline{+} h+i \lambda_{\mathrm{a}}}+\frac{F_{2}-F_{4}}{\overline{+}+i \lambda_{\mathrm{b}}}\right)\left\{J_{3}(s,-s \mp h)+J_{3}(s, s)\right\}\right\} ; \\
c_{0}= & A\left(F_{3}-F_{2}\right)\left\{\left(\frac{1}{h+i \lambda_{\mathrm{a}}}+\frac{1}{h+i \lambda_{\mathrm{b}}}\right)\left\{J_{3}(s,-s)+J_{3}(s, s+h)\right\}\right. \\
+ & \left.\left(\frac{1}{-h+i \lambda_{\mathrm{a}}}+\frac{1}{-h+i \lambda_{\mathrm{b}}}\right)\left\{J_{3}(s,-s)+J_{3}(s, s-h)\right\}\right\} ; \\
c_{ \pm 1}=A\left(F_{3}-F_{2}\right)\left(\frac{1}{ \pm h+i \lambda_{\mathrm{a}}}+\frac{1}{ \pm h+i \lambda_{\mathrm{b}}}\right)\left\{J_{3}(s \pm h,-s \pm h)+J_{3}(s \pm h, s)\right\}
\end{array}
$$

where the constant $A$ and the function $J_{3}$ are defined by, respectively,

$$
\begin{aligned}
& A=\Omega \bar{N} /\left(16 \varepsilon_{0} \hbar^{3} \lambda_{d}^{3}\right) ; \\
& J_{3}\left(s^{\prime}, s^{\prime \prime}\right)=J_{1}\left(s^{\prime}, s^{\prime \prime}\right)+J_{2}\left(s^{\prime}, s^{\prime \prime}\right) .
\end{aligned}
$$

The $\alpha, \beta, \gamma$, and $\delta$ coefficients can now easily be calculated by using

$$
\begin{aligned}
& \alpha=a_{0} ; \quad \alpha_{1}=a_{0}-\frac{1}{2}\left(a_{1}+a_{-1}\right) ; \quad \beta=b_{0,0} \\
& \beta_{1}=b_{0,0}-\frac{1}{4}\left(b_{1,1}+b_{-1,-1}+b_{1,-1}+b_{-1,1}\right) ; \\
& \beta_{2}=\beta_{1}+\frac{1}{2}\left(b_{0,1}+b_{0,-1}-b_{1,0}-b_{-1,0}\right) ;
\end{aligned}
$$




$$
\begin{aligned}
\gamma=\frac{1}{2}\left(c_{0}+c_{1}+c_{-1}\right) ; \quad \gamma_{1}=\frac{1}{2}\left(c_{0}-c_{1}-c_{-1}\right) ; \\
\delta=b_{0,0}+\frac{1}{4}\left(b_{1,1}+b_{-1,-1}+b_{1,-1}+b_{-1,1}\right) \\
+\frac{1}{2}\left(b_{1,0}+b_{0,1}+b_{-1,0}+b_{0,-1}\right) .
\end{aligned}
$$

D. approximate solution of equation (7-2)

When fixed values of $I$ and $R$ are substituted, (7-2) is of the following type (assume $R \neq 0, \pi / 2$ ):

$$
\mathrm{a}_{t} \theta=C-A \sin 2 \theta-B \cos 2 \theta \text {. }
$$

By switching to the variable $\tan \theta$ this equation can be transformed into

$$
\mathrm{d}_{t} \tan \theta=(B+C) \tan ^{2} \theta-2 A \tan \theta-B+C
$$

which can be solved analytically. Stable, time-independent solutions are found to exist whenever at least one of the following conditions is satisfied:

$$
\begin{aligned}
& \text { (a) } C^{2}-A^{2}-B^{2} \leq 0 \text {; } \\
& \text { (b) } B+C=0 .
\end{aligned}
$$

In all other cases the solution for $\theta$ is given by

$$
\tan \Theta=(B+C)^{-1}\left\{A+W \tan \left(W t+\psi_{0}\right)\right\},
$$

where $W$, identified as the fundamental difference frequency, is defined as

$$
W=\left(C^{2}-A^{2}-B^{2}\right)^{\frac{1}{2}}
$$

and where $\psi_{0}$ is a constant of integration.

An explicit expression for $\theta$ as a function of time can be obtained 
from (D-3). By substitution it can easily be verified that

$$
\theta=\operatorname{sgn}(B+C)\left\{W t+F(t)+\psi_{1}\right\}
$$

is just such an expression, where

$$
\begin{aligned}
& F(t)=\arctan \left(\frac{A+(W-|B+C|) \tan \left(W t+\psi_{1}\right)}{|B+C|+A \tan \left(W t+\psi_{1}\right)+W \tan ^{2}\left(W t+\psi_{1}\right)}\right) ; \\
& \operatorname{sgn}(B+C)=\left\{\begin{array}{l}
+1 \text { if } B+C>0 \\
-1 \text { if } B+C<0
\end{array},\right.
\end{aligned}
$$

and where $\psi_{1}$ is a constant of integration. According to (D-5), the time evolution of $\theta$ has a uniform part, corresponding to the fundamental frequency $W$, superimposed by an alternating part $F(t)$, oscillating nonsinusoidally at twice the fundamental frequency. 


\section{LIST OF CONVENTIONS AND FREQUENTLY USED SYMBOLS}

1 conventions

$\partial_{\xi}=\frac{\partial}{\partial \xi} \quad$ (partial) differentiation with respect to $\xi$ ( $\xi$ is a real variable)

$\partial_{\xi}^{2}=\frac{\partial^{2}}{\partial \xi^{2}}$

$d_{t}=\frac{d}{d t} \quad$ total time derivative

$\underline{\nabla}=\underline{\hat{x}}^{\partial}{ }_{x}+\underline{\hat{y}}_{\partial} y+\underline{\hat{z} \partial} z$

$\delta_{\xi \eta}=\left\{\begin{array}{l}1 \text { if } \xi=n \\ 0 \text { if } \xi \neq n\end{array} \quad(\xi, n\right.$ are real variables $)$

$\delta\left(n, n^{\prime}\right)=\left\{\begin{array}{l}1 \text { if } n=n^{\prime} \\ 0 \text { if } n \neq n^{\prime}\end{array} \quad\left(n, n^{\prime}\right.\right.$ are integers $)$

$\pi$ : pi $(3.14159 \ldots)$

i : imaginary unit $(\sqrt{ }-1)$

* : complex conjugation

c.c.: complex conjugation

arg : argument (of complex number)

$\sum$ : summation

- : usual symmetric scalar product

$x$ : usual antisymmetric vector product

ه : symmetric tensor product [definition in (3-9)]

† : hermitian conjugation

$[$,$] : commutator ([A, B] \equiv A B-B A$, where $A$ and $B$ are operators $)$

$\operatorname{Tr}:$ trace $\left(\operatorname{Tr} A \equiv \sum_{l} A_{2 l}\right.$, where $A$ is an operator) 
Primes are used in two different ways:

If $A$ is a complex-valued quantity, then we will sometimes write $A=A^{\prime}+i A^{\prime \prime}$, where $A^{\prime}$ and $A^{\prime \prime}$ are real. Then $A^{\prime}$ is the real part of $A$ and $A^{\prime \prime}$ is the imaginary part.

If $B$ is a real variable, then $B^{\prime}, B^{\prime \prime}$ or $B^{\prime \prime \prime}$ is used to distinguish between different values of the same variable.

\section{Latin symbols}

symbol

description

first appearance or definition in

$a, a_{1}, a_{n}$
$a_{\eta}, a_{\eta}^{n}$
$a_{x}, a_{z}$
$b, \underline{b}$
$b, b_{n n^{\prime}}$
$b_{\eta, Z}$,
$b_{x x}, b_{z z}$,
$b_{x z}, b_{z x}$
$c$
$c, c_{n n}$
$c_{\eta}$
$c_{x}, c_{z}$
$d$
$d_{n n}$,

first-order constitutive

$(2-54) ;(6-4)$

first-order constitutive coefficient

$(2-40)-(2-42) ;(5-54)$

first-order constitutive coefficient

impact parameter

$(4-24)$

third-order constitutive coefficient

$(2-54) ;(6-4)$

third-order constitutive

$(2-40)-(2-42) ;(5-55)$ coefficient

third-order constitutive

$(2-59)$ coefficient

velocity of light in free space (2-3)

third-order constitutive

coefficient

$(2-54) ;(6-4)$

third-order constitutive

coefficient

$(2-40)-(2-42)$

third-order constitutive

coefficient

density of atoms

$(3-63)$

third-order constitutive

coefficient 
vector operator proportional to

$\underline{\hat{D}}$

the electric-dipole moment operator

the displacement current operator

$\frac{D}{\hat{D}}$

expectation value of $D$

expectation value of $\underline{\hat{D}}$

$\underline{E}, \underline{E}(\underline{r}, t) \quad$ space and time dependent electric field

$E, E(t)$ weakly time-dependent electric-field amp1itude

$\underline{E}$

weakly time-dependent electric-field vector amplitude

$E_{1}, E_{0}, E_{-1}$

circular components of electric-

$F_{1}, F_{2}, F_{3}, F_{4}$ field vector amplitude

$(5-37)-(5-40)$

H, $\mathrm{H}$

$\mathrm{H}_{0}$

$H_{i}$

h

dimensionless quantities characteristic

for the type of transition

magnetic field vector and magnitude

Section 2.5

hamiltonian of unperturbed atom

$(3-7)$

interaction hamiltonian

(3-14)

Zeeman-splitting frequency normalized to

$(5-49)$

the Doppler width

$\hbar$

Planck's constant divided by $2 \pi$

Section 3.1

$I, I_{n}$

polarization parameter proportional to the intensity

$\underline{J}$

vector operator proportional to the

angular-momentum operator

J expectation value of $\underline{J}$

$(2-32) ;(6-2)$

$(3-3)$

(3-24) $J_{0}, J_{q}, J_{q}^{\alpha} \quad \begin{aligned} & \text { dimensionless functions obtained after } \\ & \text { velocity integration }\end{aligned}$

j

tota1-angular-momentum quantum number

Section 1.1

$k, k_{n}, k \quad$ wave vector (number)

$(3-41) ;(5-5)$

$k_{10}$

wave number corresponding to transition

$(4-21)$

frequency

$L$

total resonator length

Fig. 2.3

velocity-dependent lorentzian

(3-61)

pump strength

$(3-70)$

global-population operator

$(3-2)$

expectation values of $N_{a}, N_{b}$ 


$\overline{\mathrm{N}}_{\mathrm{ab}}$
$\underline{\mathrm{P}}, \underline{\mathrm{P}}(\underline{r}, t)$
$P, P(t)$
$\underline{P}$
$P_{1}, P_{0}, P_{-1}$
$P$
$\stackrel{?}{P}$

population difference

(3-54)

space and time dependent electric polarization

weakly time-dependent polarization amplitude

weakly time-dependent polarization vector amplitude

circular components of polarization vector amplitude

reduced matrix element

$(3-13)$;

$(5-9),(5-10)$

tensor operator proportional to the

quadrupole-momentum operator

expectation value of $\cong$

departure of the density matrix from equilibrium

polarization parameter

position vector

(2-12)

reflection matrix

detuning frequency normalized to the Doppler width

time coordinate

l-th natural mode

normalized electric perturbation

normalized electric perturbation amplitude

velocity of an atom

difference frequency

Section 2.5

$x, y, z$

space coordinates

respective unit vectors in $x, y$ and $z$ direction

$$
\begin{aligned}
& \alpha, \alpha_{1} \\
& \beta, \beta_{1}, \beta_{2} \\
& \Gamma^{\prime}, \Gamma^{\prime \prime} \\
& \Gamma_{a}, \Gamma_{p} \\
& \gamma, \gamma_{1}
\end{aligned}
$$

first-order constitutive coefficient

third-order constitutive coefficient

isotropic resonator loss and phase shift

$(\mathrm{B}-2)$

magnitude of 1.a.a. and 1.p.a.

$(\mathrm{B}-3),(\mathrm{B}-4)$

third-order constitutive coefficient 


\begin{tabular}{|c|c|c|}
\hline$\delta$ & third-order constitutive coefficient & $(2-63)$ \\
\hline$\varepsilon_{0}$ & permittivity of vacuum & $(2-6)$ \\
\hline . & space coordinate & $\mathrm{Fig} \cdot 2 \cdot 3$ \\
\hline & space coordinate & Fig. 2.3 \\
\hline$n_{e}, n_{0}$ & excitation density & $(4-8) ;(6-27)$ \\
\hline$\theta, \theta_{n}$ & polarization parameter & $(2-34)$ \\
\hline a & $\begin{array}{l}\text { angle specifying the low-loss main axis } \\
\text { of l.a.a }\end{array}$ & $(B-3)$ \\
\hline$\theta_{\mathrm{p}}$ & $\begin{array}{l}\text { angle specifying the low-frequency main } \\
\text { axis of } 1 . p \cdot a \text {. }\end{array}$ & $(B-4)$ \\
\hline$\lambda_{\eta}, \lambda_{n, q}$ & decay constants of free oscillations & $(2-2) ;(A-8)$ \\
\hline$\lambda_{a}, \lambda_{b}, \lambda_{J}, \lambda_{D}$ & relaxation constants of atomic observables & $\begin{array}{l}(3-28)-(3-33) ; \\
(3-55)\end{array}$ \\
\hline$\lambda_{\mathrm{sp}}^{D}$ & inverse life time for spontaneous emission & $(4-22)$ \\
\hline$\lambda_{\mathrm{d}}$ & Doppler width & $(5-48)$ \\
\hline$x$ & $\begin{array}{l}\text { relaxation constant normalized to the } \\
\text { Doppler width }\end{array}$ & $(5-49)$ \\
\hline$\underline{\mu}$ & electric-dipole operator & $(3-12) ;(5-6)$ \\
\hline$v$ & Zeeman splitting frequency & $(5-4)$ \\
\hline$\xi$ & space coordinate & Fig.2.3 \\
\hline$\rho$ & density matrix & $(3-16) ;(5-12)$ \\
\hline$\sigma$ & dimensionless collision parameter & $(4-26)$ \\
\hline$\sigma_{n, q}$ & eigenvector of reflection matrix & $(A-11)$ \\
\hline$\tau$ & dimensionless collision parameter & $(4-25)$ \\
\hline$u, u_{n}$ & polarization parameter (azimuth) & $(2-35)$ \\
\hline$\Phi(\underline{v})$ & velocity distribution function & $(3-63)$ \\
\hline$\phi_{n}, \phi_{n, q}$ & frequency shift with respect to $n \pi c / L$ & $(2-13) ;(2-22)$ \\
\hline$x, x_{n}$ & polarization parameter (ellipticity) & $(2-35)$ \\
\hline 0 & atomic transition frequency & $(3-20)$ \\
\hline & real frequency & $(2-2)$ \\
\hline & complex frequency & $(2-2)$ \\
\hline
\end{tabular}


132 


\section{REFERENCES}

[1] M. Sargent III, M.0. Scully, W.E. Lamb Jr., LASER PHYSICS, AddisonWesley, London (1974), Chapter 4

[2] 0. Sve1to, PRINCIPLES OF LASERS, Plenum Press, New York (1976)

[3] R.H. Pantel1, H.E. Puthoff, FUNDAMENTALS OF QUANTUM ELECTRONICS, Wiley and Sons, New York (1969), Chapter 6

[4] M. Sargent III, M.O. Scully, W.E. Lamb Jr., LASER PHYSICS, AddisonWesley, London (1974), Chapter 14

[5] A. Javan, W.R. Bennett Jr., D.R. Herriot, Phys. Rev. Lett. $\underline{6}$ (1961) 106

[6] A.L. Schawlow, C.H. Townes, Phys. Rev. 112 (1958) 1940

[7] W.R. Bennett Jr., Phys. Rev. 126 (1962) 580

[8] H. Statz, R. Paananen, G.F. Koster, Journ. App1. Phys. 33 (1962) 2319

[9] C.L. Tang, H. Statz, Phys. Rev. 128 (1962) 1013

[10] R.L. Fork, C.K.N. Patel, App1. Phys. Lett. $\underline{2}$ (1963) 180

[11] R. Paananen, C.L. Tang, H. Statz, Proc. IEEE 51 (1963) 63

[12] H. De Lang, G. Bouwhuis, Phys. Lett. 7 (1963) 29

[13] M. Dumont, G. Durand, Phys. Lett. 8 (1964) 100

[14] W. Culshaw, J. Kannelaud, Phys. Rev. 133 (1964) A691

[15] R.L. Fork, C.K.N. Patel, Proc. IEEE 51 (1964) 208

[16] W.F. Bel1, A.L. Bloom, Appl. Optics 3 (1964) 413

[17] W. Culshaw, J. Kannelaud, Phys. Rev. 136 (1964) A1209

[18] C.V. Heer, R.D. Graft, Phys. Rev. 140A (1965) 1088

[19] P.T. Bolwijn, App1. Phys. Lett. $\underline{6}$ (1965) 203

[20] I. Tobias, M.L. Skolnick, R.A. Wallace, T.G. Polonyi, Appl. Phys. Lett. 6 (1965) 198

[21] R.L. Fork, M. Sargent III, Phys. Rev. 139 (1965) A617

[22] H. De Lang, G. Bouwhuis, Phys. Lett. 19 (1965) 481

[23] W.E. Lamb Jr., Phys. Rev. 134 (1964) A1429

[24] H. De Lang, G. Bouwhuis, E.T. Ferguson, Phys. Lett. 19 (1965) 482

[25] D. Polder, W. Van Haeringen, Phys. Lett. 19 (1965) 380

[26] H. De Lang, G. Bouwhuis, Phys. Lett. 20 (1966) 383

[27] D. Polder, W. Van Haeringen, Phys. Lett. 25A (1967) 337 
[28] A. Omont, Journnal de Phys. 26 (1965) 26

[29] M.I. D'Yakonov, V.I. Perel, Sov. Phys. JETP 21 (1965) 227

[30] G. Durand, Ann. Inst. Henri Poincaré 4 (1966) 263

[31] W. Culshaw, J. Kannelaud, Phys. Rev. 145 (1966) 257

[32] M.I. D'Yakonov, V.I. Pere1, Optics and Spectr. 20 (1966) 257

[33] J. Kannelaud, W. Culshaw, Phys. Rev. 141 (1966) 237

[34] H. De Lang, Philips Res. Repts. 19 (1964) 429

[35] C.H.F. Velze1, Phys. Lett. 23 (1966) 72

[36] H. De Lang, Philips Res. Repts. Supp1. $\underline{8}$ (1967)

[37] W. Van Haeringen, Phys. Lett. 24A (i967) 65

[38] H. Pelikan, Zeitschr. f. Physik 201 (1967) 523

[39] W. Van Haeringen, Phys. Rev. 158 (1967) 256

[40] M. Sargent III, W.E. Lamb Jr., R.L. Fork, Phys. Rev. 164 (1967) 436

[41] M. Sargent III, W.E. Lamb Jr., R.L. Fork, Phys. Rev. 164 (1967) 450

[42] W.J. Tomlinson, R.L. Fork, Phys. Rev. 164 (1967) 466

[43] R.A. Settles, C.V. Heer, App1. Phys. Lett. 12 (1968) 350

[44] W. Van Haeringen, H. De Lang, Phys. Rev. 180 (1969) 624

[45] D. Lenstra, App1. Phys. 17 (1978) 257

[46] D.H. McMahon, Rev. Sci. Inst. 40 (1969) 727

[47] R.H. Morris, J.B. Ferguson, J.S. Warniack, App1. Opt. 14 (1975) 2808

[48] M.D. Crisp, Optics Comm. 23 (1976) 316

[49] R.A.J. Keijser, Optics Comm. 23 (1977) 194

[50] D. Lenstra, W. Van Haeringen, Optics Comm. 23 (1977) 311

[51] L.A. Weinstein, OPEN RESONATORS AND OPEN WAVEGUIDES, The Golem Press, Boulder (Colorado) (1969)

[52] A.G. Fox, T. Li, Bel1 System Tech. J. 40 (1961) 61

[53] H. Blok, Thesis, Rpt. nr. 1970-7, Lab. of Electromagnetic Research, Dpt. of Electrical Eng., Delft University of Technology, Delft (Netherlands) (1970)

[54] L. Ronchi, in: LASER HANDBOOK VOL. 1, North-Holland, Amsterdam (1972)

[55] B. Decomps, M. Dumont, IEEE Journ. of Q.E. QE-4 (1968) 916

[56] J.Z. Klose, Phys. Rev. 141 (1966) 181

[57] W. Happer, Rev. of Mod. Phys. 44 (1972) 169

[58] A. Omont, Progr. Q.E. $\underline{5}$ (1977) 69

[59] A. Messiah, MÉCANIQUE QUANTIQUE, Dunod, Paris (1960) 
[60] M.I. D'Yakonov, V.I. Perel, Sov. Phys. JETP 20 (1965) 997

[61] M.I. D'Yakonov, V.I. Perel, Phys. Lett. 41A (1972) 451

[62] H. Weyl, THE THEORY OF GROUPS AND QUANTUM MECHANICS, Dover Pub1. Inc., New York

[63] M. Sargent III, M.0. Scully, W.E. Lamb Jr., LASER PHYSICS, AddisonWesley, London (1974) Appendix C

[64] R. Balhorn, H. Kunzmann, F. Lebowsky, App1. Opt. 11 (1972) 742

[65] G. Mas, H. Blancher, J. Roig, App1. Opt. 13 (1974) 2771

[66] J.L. Willems, STABILITÄT DYNAMISCHER SYSTEME, R. Oldenbourg (1973) 
Dit proefschrift handelt over de theorie van polarisatie-effecten in gaslasers. De theorie betreft zowel de elektrische polarisatie in het gas, als de optische polarisatietoestand van het laserveld. Hierbij worden de effecten onderzocht tengevolge van verzadiging, uitwendig aangelegde magneetvelden en zwakke spiegelanisotropieën, al dan niet in combinatie met elkaar.

Na een herformulering van de algemene theorie van laserwerking worden de elektromagnetische veldvergelijkingen voor een gaslaser afgeleid, waarbij de nadruk ligt op het vectoriële karakter van het veld. Vanzelfsprekend bevat de theorie een gedetailleerde beschrijving van de wisselwerking tussen een elektromagnetisch veld en het (actieve) gas. Dit omvat de bestudering van de tijdevolutie van verwachtingswaarden van quantummechanische atomaire observabelen onder de invloed van een klassiek, dus niet gequantiseerd, stralingsveld. Deze benadering staat bekend als de "semi-klassieke" benadering en leidt tot een juiste beschrijving zolang niet te lage intensiteiten van het stralingsveld worden beschouwd.

In de opzet van de theorie wordt rekening gehouden met de mogelijkheden dat (a) een uitwendig magneetveld is aangelegd over (een deel van) het actieve gas en/of dat (b) de reflectie-eigenschappen van de resonatorspiegels anisotroop zijn. Speciaal de effecten tengevolge van een transversaal magneetveld, d.w.z. gericht loodrecht op de voortplantingsrichting van de elektromagnetische golf, worden in detail behandeld.

$\mathrm{Na}$ een algemene inleiding en een beknopt overzicht van de literatuur in Hoofdstuk 1, wordt in Hoofdstuk 2 de theorie van laserwerking ontwikkeld. Hierbij wordt uitgegaan van de "vrije oscillaties" van een passief resonatorsysteem. Dit zijn de discrete, exponentieel in de tijd afnemende elektromagnetische veldconfiguraties die in een gegeven resonator kunnen bestaan. De bij deze vrije oscillaties behorende ruimtelijke veldverdelingen definiëren de "natuur1ijke" modi. Laserwerking wordt beschreven als een excitatie van deze modi gedurende een tijdsinterval dat veel groter is 
dan de bijbehorende karakteristieke vervaltijden. Met het oog op latere toepassingen wordt de algemene theorie nader uitgewerkt voor resonatoren van het Fabry-Pérot type. Gebruikmakend van een ééndimensionaal model worden voor een dergelijke resonator de natuurlijke modi bepaald. Omdat het vectoriële karakter van deze modi in het geval van anisotrope spiegels volledig is vastgelegd, kan een laserveld worden gekarakteriseerd door het opgeven van een aantal scalaire grootheden, die de amplitudes aangeven waarmee de diverse modi in dit veld zijn vertegenwoordigd. Als de anisotropieën in de spiegels voldoende zwak zijn, kan eenvoudig worden aangetoond dat deze modi, afgezien van hun vectoriële karakter, twee aan twee praktisch dezelfde ruimtelijke afhankelijkheid vertonen; d.w.z. zij hebben hetzelfde longitudinale moduskarakter. In dat geval kan laserwerking worden beschreven in termen van de gebruikelijke longitudinale modi, die dan echter wel vectoriële veldamplitudes bezitten. Hierbij is elke vectoramplitude een lineaire combinatie van de twee karakteristieke polarisatievectoren van de oorspronkelijke modi.

In Hoofdstuk 3 wordt de response van het gas op een monochromatische, elektrische staande golf bestudeerd, voor een atomaire overgang tussen een triplet en een singlet niveau. Dit geval is niet alleen interessant als een inleiding tot de meer algemene en ingewikkelde gevallen die worden behandeld in Hoofdstuk 5; de afzonderlijke behandeling ervan stelt ons tevens in staat te laten zien dat verschillen in bepalde relaxatiesnelheden hier een belangrijke rol spelen. In Hoofdstuk 4 wordt hierop aangesloten door de verkregen resultaten toe te passen op het probleem van de door het medium geïnduceerde polarisatievoorkeuren in een gaslaser, waarin één longitudinale modus is geëxciteerd. Een berekening van het effect van interatomaire botsingen op de relaxatiesnelheden leidt tot de conclusie dat theorie en experiment goede overeenkomst vertonen.

In Hoofdstuk 5 wordt de response van een gas op een multimodaal elektromagnetisch veld afgeleid voor een grote klasse van atomaire overgangen. Hierbij wordt de aanwezigheid verondersteld van een uitwendig aangelegd magneetveld. De door het medium geînduceerde polarisatievoorkeuren in een gaslaser in de afwezigheid van magneetvelden worden besproken in Hoofdstuk 6 , zowel voor het unimodale als voor het bimodale geval. 
Het blijkt dat in het laatste geval de wisselwerking tussen de modi van zodanige aard is, dat beide modi in het algemeen streven naar een toestand met orthogonale polarisatievectoren. Hierna wordt het effect van twee speciale resonatoranisotropieën behandeld voor een laser waarvan het actieve medium een voorkeur vertoont voor lineaire polarisatié.

In Hoofdstuk 7 wordt het polarisatiegedrag van gaslasers in transversale magneetvelden onderzocht voor het geval waarin éên enkele longitudinale modus is geëxciteerd. De theorie wordt hier geconfronteerd met een aantal experimenten in transversale magneetvelden. Het blijkt dat onderscheid gemaakt moet worden tussen een slechts gedeeltelijk over het actieve medium aangelegd magneetveld en een magneetveld dat uniform is over het gehele actieve medium. Door hiermee rekening te houden, wordt voor een aantal experimenten een bevredigende theoretische beschrijving gegeven. In twee andere gevallen wordt goede overeenkomst tussen theorie en experiment verkregen wanneer resonatoranisotropieën van acceptabele soort en grootte in de beschouwing worden opgenomen. Tenslotte vindt een behandeling plaats van de invloed die zwakke anisotropieën kunnen hebben op het elektromagnetische veld van een in één longitudinale modus werkende gaslaser, als deze wordt gepolariseerd met behulp van een sterk transversaal magneetveld. 


\section{LEVENSBER I CHT}

Daan Lenstra werd op 26 september 1947 te Amsterdam geboren. Na zijn middelbare schoolopleiding aan de Rijks-HBS te Zwolle ging hij in 1964 naar Groningen, om daar natuurkunde te studeren. Het kandidaatsexamen legde hij af in 1969, waarna hij theoretische natuurkunde als hoofdvak koos. Zijn afstudeerwerk handelde over de algebraïsche formulering van quantumveldentheorie. In 1972 voltooide hij zijn studie.

$\mathrm{Na}$ een aanvankelijke aarzeling met betrekking tot het aanvangen van een wetenschappelijke loopbaan en het vervullen van de militaire dienstplicht, trad hij in 1975 in dienst van de TH Delft als wetenschappelijk medewerker bij de afdeling Elektrotechniek. Hier werd hij medewerker van de buitengewoon hoogleraar Prof. Dr. W. van Haeringen die sinds 1973 binnen de vakgroep Theoretische Elektriciteitsleer activiteiten ontplooit op het gebied van de quantumtheoretische grondslagen van de theoretische elektriciteitsleer. Daan is betrokken bij het onderwijs aan doctoraalstudenten en verricht onderzoek op het gebied van de elektrische geleiding in vaste stoffen en op het gebied van de interactie van straling en materie. De laatstgenoemde onderzoekingen hebben geleid tot de samenste1ling van dit proefschrift.

Naast zijn werk heeft Daan veel belangstelling voor moderne muziek en speelt hij als basgitarist in een poporkest. Een andere aanvulling op zijn abstracte studie van de dode materie vond en vindt hij in het intens ervaren van de levende natuur, zowel in zijn moestuin als tijdens wekenlange voettochten.

M. 
Het is in het algemeen incorrect een theoretische analyse van de stabiele eigentoestanden van het elektromagnetische veld in een laser uitsluitend te baseren op een "maximum gain" beschouwing.

C.L. Tang, H. Statz: "Nonlinear effects in the resonant absorbtion of several oscillating fields by a gas", Phys. Rev. 128 (1962) 1013, pagina 1019

W.J. Witteman: "Theory of mode interaction in the case of small mode spacing", Phys. Rev. 143 (1966) 316, pagina 316

\section{2}

De door Stephan et al. gehanteerde vergelijkingen voor de dichtheidsmatrix geven een principieel onjuiste beschrijving van de relaxatieverschijnselen.

G. Stephan, R. Le Naour, A. Le Floch: "Experimental and theoretical study of the anisotropy induced in a gas by a saturating field", Phys. Rev. $\underline{A 17}$ (1978) 733, vergelijkingen (7a)-(7c)

3

De aanwezigheid van een magneetveldvrij gebied in een $j=1 \rightarrow j=2$ unimodale gaslaser met isotrope spiegels kan in het geval van een transversaal magneetveld leiden tot stabilisatie van de polarisatietoestand van de modus. In het geval van een uniform axiaal magneetveld daarentegen is het enige substantiële effect bij een toenemend magneetveldvrij gebied een monotone afname van de rotatiesnelheid van het polarisatievlak.

Dit proefschrift, Hoofdstuk 7

4

In het algemeen wordt door elke monochromatische elektromagnetische golf in een isotroop medium met een gegeven dipoolovergang een anisotropie in de susceptibiliteit geinduceerd. In het geval van een doublet-doublet overgang kan men inzien dat door een lineair gepolariseerd veld slechts dan een anisotropie wordt geînduceerd indien in tenminste één van beide doubletten de globale populatie en het impulsmoment met verschillende snelheden relaxeren.

Dit proefschrift, pagina 85 
De verschillende Fockrepresentaties van de canonieke commutatierelaties die bij de beschrijving van gegeneraliseerde vrije velden (1) een rol spelen, kunnen op eenvoudige wijze worden geclassificeerd, waarbij het criterium voor de unitair-equivalentie van twee dergelijke representaties gelijkluidend is aan dat voor de canonieke anticommutatierelaties (2). Als echter aan een gegeneraliseerd vrij veld de gebruikelijke spectrumconditie (3) wordt opgelegd, dan is de representatie op unitair-equivalentie na volledig vastgelegd.

(1) O.W. Greenberg: "Generalized free fields and models of local field theory", Ann. Phys. 16 (1961) 158

(2) J. Manuceau, A. Verbeure: "The theorem on unitary equivalence of Fock representations", Ann. Inst. Henri Poincaré, 16 (1971) 87

(3) R.F. Streater, A.S. Wightman: PCT, SPIN \& STATISTICS, AND ALL THAT, W.A. Benjamin Inc., New York (1964)

6

Datgene wat de leerboeken van Peierls en Ziman te berde brengen aangaande de betekenis van Normale en Umklapp processen is op zijn minst onzorgvuldig geformuleerd. De beschrijving gegeven door Taylor voldoet weliswaar beter, maar ook deze auteur benadrukt nog te weinig dat het onderscheid tussen deze processen in feite artificieel is.

R.E. Peierls: QUANTUM THEORY OF SOLIDS, Clarendon Press, Oxford (1955), pagina's 41,42

J.M. Ziman: PRINCIPLES OF THE THEORY OF SOLIDS, University Press, Cambridge (1972), pagina 60

P.L. Taylor: A QUANTUM APPROACH TO THE SOLID STATE, Prentice Hall, Inc., Englewood Cliffs, New Jersey (1970), pagina 105

7

Berekening van de soortelijke weerstand van een polyvalent metaal volgens een methode waarin de verdelingsfunctie van de elektronen in aanwezigheid van een elektrisch veld wordt berekend, verdient de voorkeur boven de veelal gebruikte variatiemethode.

H.M. de Ruiter, W. van Haeringen, D. Lenstra: "Some remarks on the use of Ziman's variational expression for the resistivity of a metal", te verschijnen in Delft Progr. Rpt. (1979)

Y. Bergman, M. Kaveh, N. Wiser: "Explanation for the deviations from Matthiessen's rule for the low-temperature electrical resistivity of the simple metals", Phys. Rev. Lett. 32 (1974) 606 
Zij $N(k)$ het maximale aantal munten waarvan er ten hoogste éen een gewicht heeft dat afwijkt van dat van alle andere en waarvoor in $k$ maal wegen met een balans kan worden bepaald

(a) of er een afwijkend gewicht voorkomt, en zo ja,

(b) welke munt dit betreft en

(c) of deze zwaarder dan wel lichter is dan de overige.

Er geldt dan $N(k)=\frac{1}{2}\left(3^{k}-3\right)$, voor $k=2,3, \ldots$

9

Zowe 1 het toestaan aan automobilisten tot het voeren van mistachterlichten als de verplichting tot het voeren van dimlicht binnen de bebouwde kom kan leiden tot een grotere onveiligheid van de fietser. De invoering van verplichte reflectoren voor deze laatste groep van weggebruikers zal hieraan weinig veranderen.

D. Lenstra

Delft, 12 september 1979 
